\title{
Performance Improvement of High-speed Edm and Ecm Combined Process by Using a Helical Tube Electrode With Matched Internal and External Flushing
}

\author{
Lei Ji \\ Nanjing Tech University \\ Yan Zhang ( $\square$ zhangyanzy@njtech.edu.cn ) \\ Nanjing Tech University \\ Guoqian Wang \\ Nanjing Tech University \\ Jie Zhang \\ Nanjing Tech University \\ Wentao Yang \\ Nanjing Tech University
}

\section{Research Article}

Keywords: high-speed electrical discharge machining, electrochemical machining, helical tube electrode, internal and external flushing, removing debris

Posted Date: March 24th, 2021

DOl: https://doi.org/10.21203/rs.3.rs-331366/v1

License: (c) (i) This work is licensed under a Creative Commons Attribution 4.0 International License.

Read Full License

Version of Record: A version of this preprint was published at The International Journal of Advanced Manufacturing Technology on August 6th, 2021. See the published version at https://doi.org/10.1007/s00170-021-07595-1. 
Performance improvement of high-speed EDM and ECM combined process by using a helical tube electrode with matched internal and external flushing

Lei Ji ${ }^{1}$, Yan Zhang ${ }^{1 *}$, Guoqian Wang ${ }^{1}$, Jie Zhang ${ }^{1}$, Wentao Yang ${ }^{1}$

1 School of Mechanical and Power Engineering, Nanjing Tech University, Nanjing 211800, PR China

TEL: 86-25-58139346

Fax: 86-25-58139346

Email: zhangyanzy@njtech.edu.cn(corresponding author) 


\begin{abstract}
Micro-hole fabrication at a high speed and accuracy of machining while maintaining high surface quality is challenging. A core difficulty is the removal of the products of machining from extremely narrow gaps. To solve this problem, this study proposes an approach that combines high-speed electrical discharge machining (EDM) with electrochemical machining (ECM) by using a helical tube electrode with matched internal and external flushing. During high-speed electrical discharge drilling, matching the internal flushing with the clockwise rotation of the helical electrode can help remove debris from the bottom of the blind hole. During ECM, matching the external flushing with the anticlockwise rotation of the helical electrode can improve the flow of electrolyte in the gap. First, the flow field was simulated to show that matching the internal and external flushing of the helical electrode can enhance the flow of the medium and reduce particle concentration in extremely narrow gaps. Second, a series of experiments were conducted to verify that the taper of the hole and the surface quality of its wall can be improved by using the helical tube electrode. Finally, an experiment was carried out to optimize the machining parameters, and yielded a minimum taper of 0.008 at a speed of rotation of $460 \mathrm{rpm}$, and pressures of internal and external flushing of $9 \mathrm{MPa}$ and $4 \mathrm{MPa}$, respectively.
\end{abstract}

Keywords: high-speed electrical discharge machining; electrochemical machining; helical tube electrode; internal and external flushing; removing debris.

\title{
1 Introduction
}

In the context of aviation manufacturing, a large number of microscopic cooling holes are present in films on the surfaces of the turbine blades of aero-engines [1-3], and these micro-holes are distributed on the blades at different angles [4-6]. Such a structure can be used to form a film of cooling gas on the surface of the blade to ensure 
its reliable operation in the ultra-high-temperature environment of the combustion chamber [7-9]. Such micro-holes are commonly made of superalloys that are highly temperature resistant, hard, and strong, such as nickel-based [10], cobalt-based [11], and titanium alloys [12]. These holes need to be machined at a high speed and accuracy while maintaining high surface quality [13]. Therefore, the machining of such filmcooling holes is a key issue in aerospace manufacturing. Combining high-speed electrical discharge drilling with electrochemical machining (ECM) is promising in this context due to such advantages as a high speed and accuracy of machining, and the ability to avoid surface defects and residual stress [14-16]. In this combined process, high-speed electrical discharge machining is first used to efficiently and accurately drill the holes, and ECM is then combined with it in situ to remove surface defects $[17,18]$. However, in case of extremely narrow machining gaps, the removal of the products of machining is a significant problem that directly influences performance $[19,20]$.

A considerable amount of research has been devoted to solving the problem of removing machining products from narrow gaps on the surface of materials. On the one hand, some scholars have attempted to improve the flow rate of the working fluid in the gap by changing the internal and external morphologies of the electrode. For example, by studying the wear in tube and rod electrodes subjected to micro-EDM drilling, Pham et al. [21] concluded that using the tube electrode was more conducive to removing debris in the narrow gap. Zhang et al. [22] noted that using a double-hole tube electrode can yield cooling holes with a high material removal rate (MRR) and precision as well as a small taper hole in case of high-speed electrochemical discharge drilling. Fang et al. [23] studied electrochemical machining in case of the vibrations of a ribbed wire electrode at a large amplitude, and verified that this enhanced the electrolyte renewal of and bubble removal from the electrode. To better remove products from the gap and 
thus improve the accuracy and efficiency of machining, Zou et al. [24] proposed wire electrochemical micro-machining using a cutting edge tool electrode. Plaza et al. [25] showed that using a helical rod electrode with micro-EDM drilling can help remove debris from narrow gaps. To reduce the entrance diameter of the hole, Yang et al. [26] used a spherical tool electrode in electrochemical discharge machining that expedited the removal of the product. On the other hand, certain scholars have examined ways to enhance the flow of the medium in the narrow gap by changing the mode of flushing. Wang et al. [27] proposed electrochemical drilling with the vacuum-based extraction of the electrolyte to accelerate reverse flow in electrochemical drilling. To distribute the flow of the electrolyte more uniformly, Zhu et al. [28] used reverse electrolyte flow with a tube electrode in electrochemical drilling to efficiently generate multiple holes of good quality. Li et. al. [29] proposed a high-speed self-adjusting electrical discharge machining/electrochemical machining approach. They applied two independent flushing systems that could switch automatically in the machining process to ensure the continuous flushing of the working fluid that improved the status of the discharge gap. Zhang et al. [30] proposed drilling film-cooling holes by using a combination of EDM/ECM in situ through the internal and side-flushing of a tubular electrode. They used a tubular electrode and a specially designed flushing device. Internal and sideflushing were performed simultaneously so that EDM and ECM could be combined in situ. The results of experiments showed this approach can improve the surface quality of holes and remove the recast layer.

To better remove machining products from narrow gaps, this study proposes matching internal and external flushing with the rotations of a helical tube electrode in a combination of high-speed EDM and ECM. First, high-speed electrical discharge drilling is used to quickly remove the material of the workpiece, which enhances 
machining efficiency. ECM is used to repair holes on the surface to remove surface defects. Second, the rotation of the helical electrode promotes fluid flow in the narrow gap to accelerate the removal of debris. Finally, different flushing strategies are used in different stages of processing. During high-speed EDM, matching the internal flushing with the clockwise rotation of the helical electrode is beneficial for removing debris from the bottom of the blind hole. During ECM, matching the external flushing with the anticlockwise rotation of the helical electrode is conducive to electrolyte flow in the gap. Therefore, this method can help accelerate the renewal of the working fluid in a combination of high-speed EDM and ECM.

We first compared the cloud patterns of the velocities of cylindrical and helical tube electrodes using a flow field simulation. The rotations of tube electrodes of varying structures were then matched with internal and external flushing in a combination of high-speed EDM and ECM in verification experiments. The effectiveness of this method was confirmed by measuring the results of machining and through observations of the holes in the wall. Finally, an experiment to optimize the parameters of machining was carried out using the proposed method, and influence of the rotating speed of the tool electrode, pressure of the working liquid, and the distance between the workpiece and the guide device was examined.

2 Principle of combination of high-speed EDM and ECM using a helical tube electrode with matched internal and external flushing 

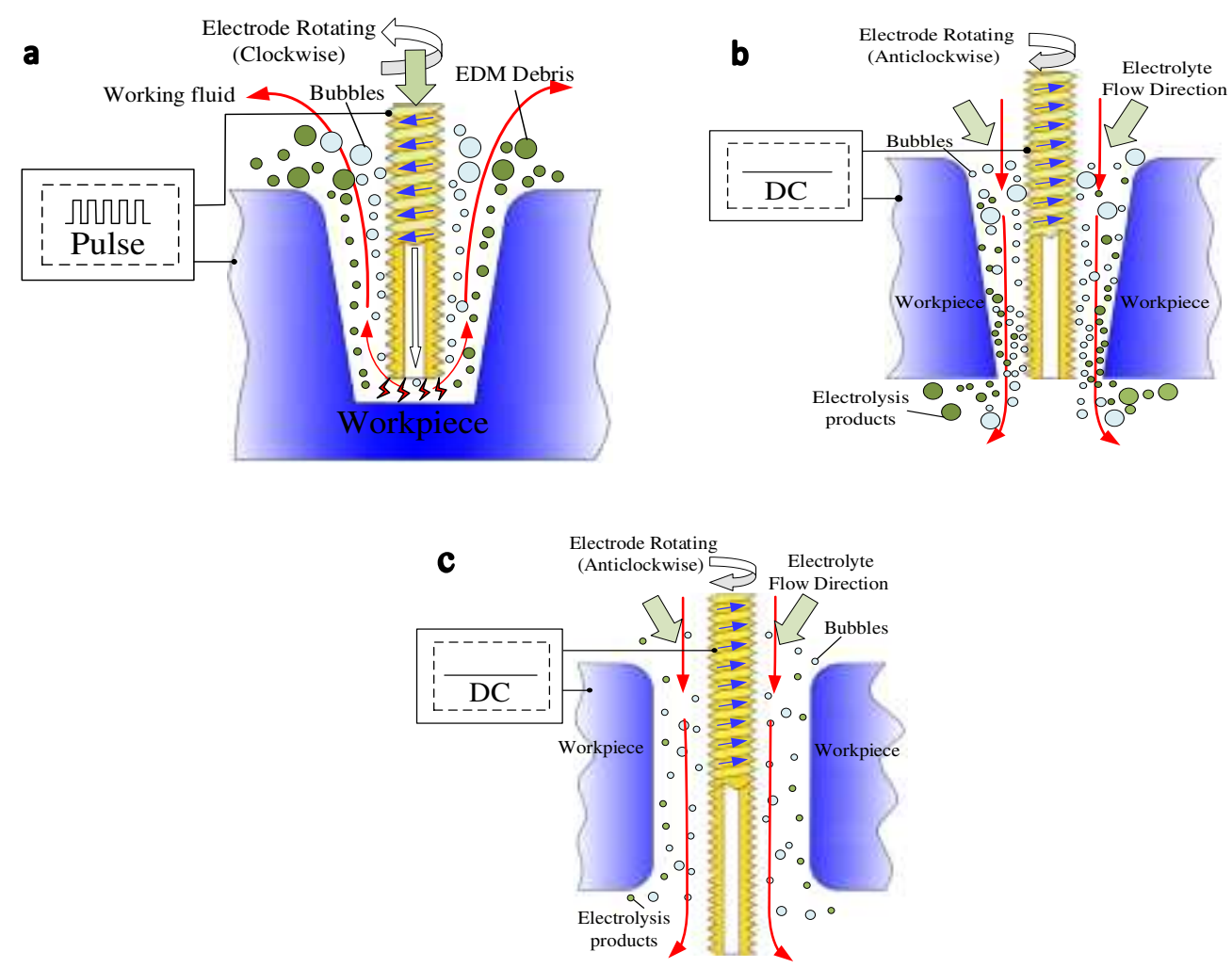

Fig. 1 Principle of the combination of high-speed EDM and ECM: (a) high-speed electrical discharge drilling; (b) ECM; (c) final results of machining

Fig. 1 shows the principle of machining using a combination of high-speed EDM and ECM. A tube electrode with a helical structure is used to enhance the flow of the medium in the gap, which is conducive to removing debris and bubbles during both types of machining.

high-speed EDM features electrical discharge drilling with the helical electrode rotating clockwise. As is shown in Fig.1(a), the rotation of the electrode can improve the uniform distribution of debris and bubbles in the gap, which reduces the accumulation of debris in narrow gaps. The helical electrode also speeds-up the flow of the medium due to the disturbance it creates. To promote the upward movement of the working fluid in the gap, the helical electrode must be rotated clockwise. Deionized water is used as the working fluid through the internal hole of the tube electrode. The internal flush mode can quickly remove debris at the bottom of the hole to significantly 
increase machining speed in the direction of drilling. However, due to the tube electrode wear and the time difference between the entrance and exit in machining, there is a taper in the hole after this procedure.

ECM features electrochemical reaming with the helical electrode rotating anticlockwise. Fig. 1(b) shows that DC power, instead of pulsed power, is applied in ECM, while the relative positions of the tool electrode and workpiece are maintained after high-speed EDM. $\mathrm{NaNO}_{3}$ is supplied to the machining gap through external flushing. Following this, the electrode rotating anticlockwise can be used to enhance the flow of the medium to ensure adequate electrolyte supply in the narrow gap. Because the material around the exit is close to the electrode, the electrochemical reaction at the exit is greater than that at the entrance, and can reduce the tapering of the holes. Within a certain electrolytic time, holes with a free taper can be obtained as shown in Fig. 1(c). Thus, micro-holes with few defects and small tapers can be produced by a combination of high-speed EDM and ECM using a helical tube electrode with matched internal and external flushing.

\section{Simulation and experimental procedure}

\subsection{CFD simulation model}

Fig. 2 shows CFD models of the blind hole stage and the breakthrough hole stage in the combination of high-speed EDM and ECM by using a helical tube electrode with matched internal and external flushing. Figs. 2(a) and 2(b) show models of the highspeed EDM stage, and Figs. 2(c) and 2(d) shows those of the ECM stage. The boundary conditions of the model were set with reference to the results of machining and conditions of the relevant tools. The parameters used in the simulation models are listed in Table 1. Based on the pressure separation solver, the steady-state turbulence k- $\varepsilon$ equation is used to calculate the CFD model. The equation expression is as follows: 


$$
\begin{aligned}
& \frac{\partial}{\partial t}(\rho k)+\frac{\partial}{\partial x_{i}}\left(\rho U_{i} k\right)=\frac{\partial}{\partial x_{j}}\left(\left(\mu+\frac{\mu_{t}}{\sigma_{k}}\right) \frac{\partial k}{\partial x_{j}}\right)+G_{k}-Y_{k}+S_{k} \\
& \frac{\partial}{\partial t}(\rho \varepsilon)+\frac{\partial}{\partial x_{i}}\left(\rho U_{i} \varepsilon\right)=\frac{\partial}{\partial x_{j}}\left(\left(\mu+\frac{\mu_{t}}{\sigma_{\varepsilon}}\right) \frac{\partial \varepsilon}{\partial x_{j}}\right)+c_{\varepsilon 1} \frac{\varepsilon}{k}\left(G_{k}+c_{\varepsilon 3} G_{b}\right)-c_{\varepsilon 2} \rho \frac{\varepsilon^{2}}{k}+S_{\varepsilon}
\end{aligned}
$$

where, $G_{k}$ is the turbulent kinetic energy generated by the laminar velocity gradient, $G_{b}$ is the turbulent kinetic energy generated by the buoyancy, $Y_{k}$ is the fluctuation caused by the transition diffusion in the recompressible turbulence, $c_{\varepsilon 1}, c_{\varepsilon 2}$ and $c_{\varepsilon 3}$ are constants, $\sigma_{k}$ and $\sigma_{\varepsilon}$ are the turbulent Prandtl numbers of equations k and $\varepsilon$, and $S_{k}$ and $S_{\varepsilon}$ are user-defined.

In the flow field simulation analysis, the liquid phase is a continuous and incompressible fluid, and the working liquid medium conforms to the law of conservation of mass in the processing process. The motion differential equation of the incompressible fluid can be expressed as:

$$
\frac{\partial u_{x}}{\partial x}+\frac{\partial u_{y}}{\partial y}+\frac{\partial u_{z}}{\partial z}=0
$$

In the processing of any volume of fluid, the momentum change rate of the fluid is equal to the sum of the mass force and stress acting on the volume. The differential form of the momentum equation is as follows:

$$
\frac{\partial(\rho v)}{\partial t}+\operatorname{div}(\rho v v)-\rho F-\operatorname{div} P=0
$$

where, $\rho \frac{D v}{D t}$ represents the inertia force per unit volume, $\rho F$ the mass force per unit volume, and $\operatorname{div} P$ the divergence of the stress tensor on the indistinguishable body.

In the Cartesian coordinate system, the motion equation expressed by stress is expressed as follows:

$$
\left\{\begin{array}{l}
\rho\left(\frac{\partial u}{\partial t}+u \frac{\partial u}{\partial x}+v \frac{\partial u}{\partial y}+w \frac{\partial u}{\partial z}\right)=\rho F_{x}+\frac{\partial p_{x x}}{\partial x}+\frac{\partial p_{x y}}{\partial y}+\frac{\partial p_{x z}}{\partial z} \\
\rho\left(\frac{\partial v}{\partial t}+u \frac{\partial v}{\partial x}+v \frac{\partial v}{\partial y}+w \frac{\partial v}{\partial z}\right)=\rho F_{y}+\frac{\partial p_{y x}}{\partial x}+\frac{\partial p_{y y}}{\partial y}+\frac{\partial p_{y z}}{\partial z} \\
\rho\left(\frac{\partial w}{\partial t}+u \frac{\partial w}{\partial x}+v \frac{\partial w}{\partial y}+w \frac{\partial w}{\partial z}\right)=\rho F_{z}+\frac{\partial p_{z x}}{\partial x}+\frac{\partial p_{z y}}{\partial y}+\frac{\partial p_{z z}}{\partial z}
\end{array}\right.
$$

Where, $u, v$ and $w$ are the components of the fluid particle velocity in $x, y$ and $z$ directions, $P$ represents the stress tensor. 
a

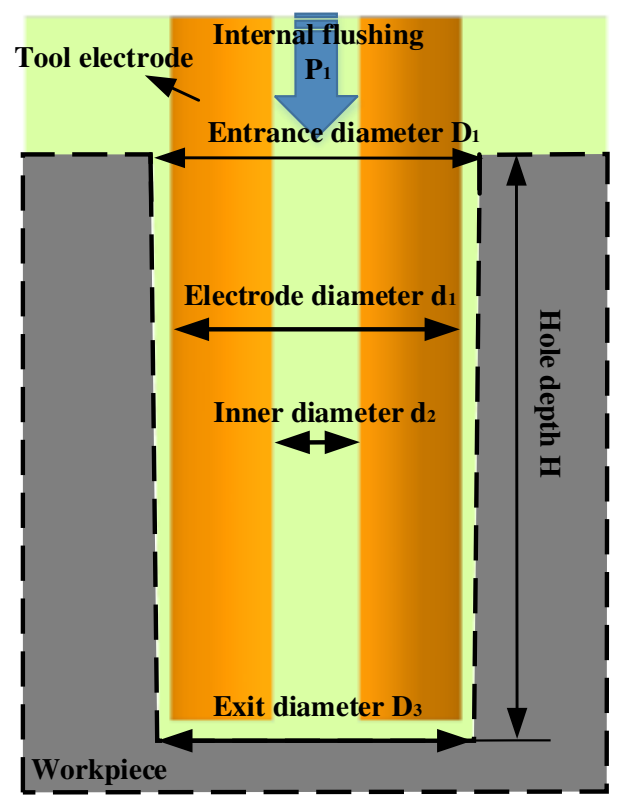

c

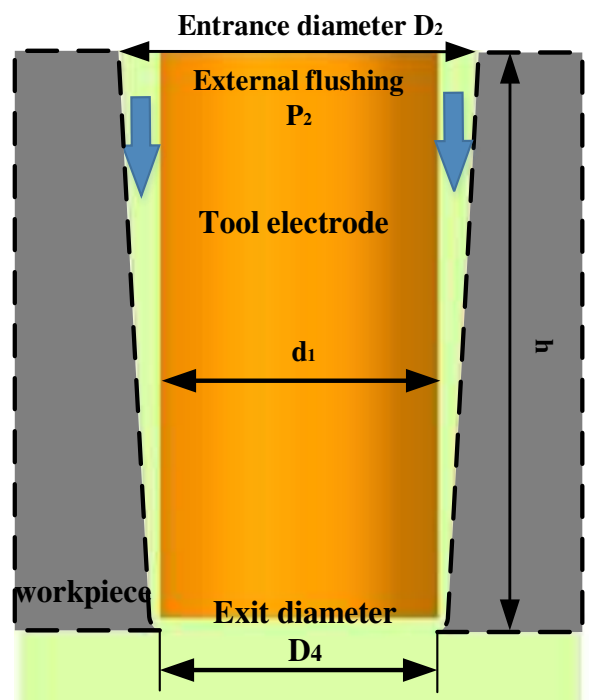

b

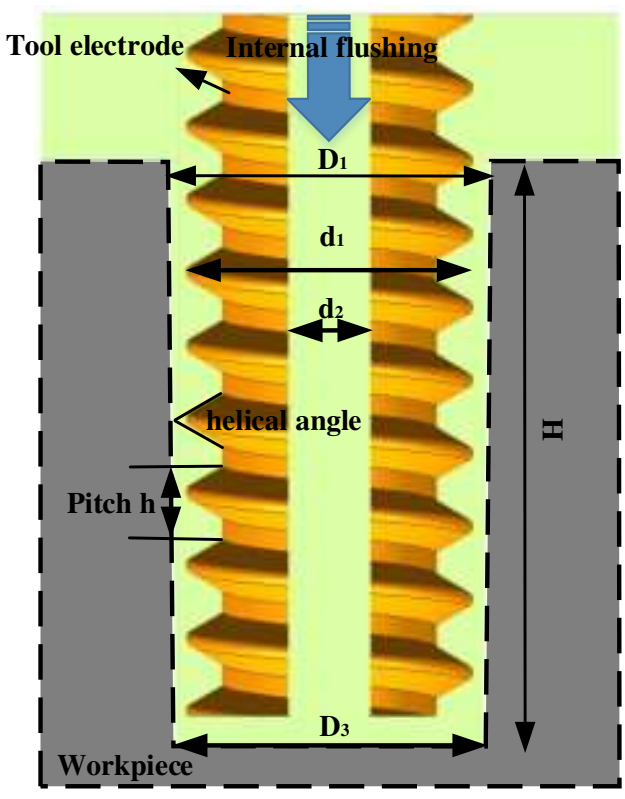

d

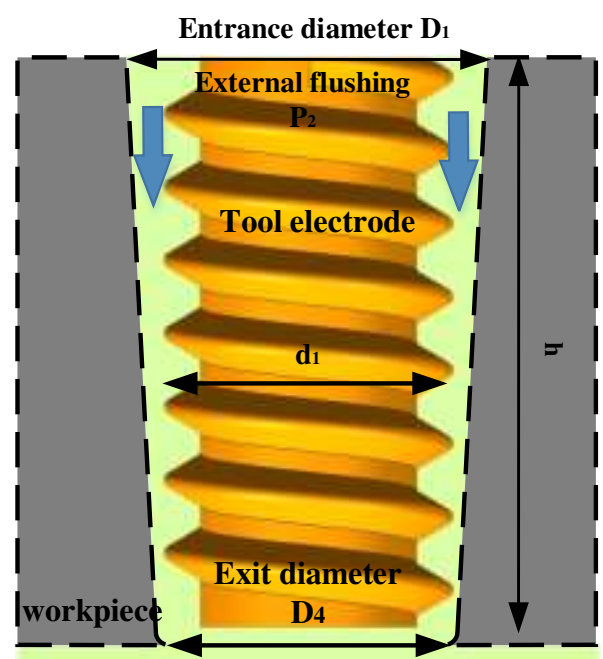

Fig. 2 CFD models of the blind hole stage and breakthrough hole stage: (a) and (c) cylindrical tube electrode; (b) and (d) helical tube electrode 


\section{Table 1}

Parameters used in the CFD model

\begin{tabular}{ll}
\hline Parameter & Values \\
\hline Electrode diameter $\left(\mathrm{d}_{1}\right)$ & $1 \mathrm{~mm}$ \\
Inner diameter $\left(\mathrm{d}_{2}\right)$ & $0.3 \mathrm{~mm}$ \\
Entrance diameter $\mathrm{D}_{1}$ & $1.12 \mathrm{~mm}$ \\
Entrance diameter $\left(\mathrm{D}_{2}\right)$ & $1.26 \mathrm{~mm}$ \\
Exit diameter $\left(\mathrm{D}_{3}\right)$ & $1.08 \mathrm{~mm}$ \\
Exit diameter $\left(\mathrm{D}_{4}\right)$ & $0.96 \mathrm{~mm}$ \\
Hole depth $(\mathrm{H})$ & $2 \mathrm{~mm}$ \\
Pitch $(\mathrm{h})$ & $0.25 \mathrm{~mm}$ \\
Helical angle & $60^{\circ}$ \\
\hline
\end{tabular}

\subsection{Experimental apparatus}

Fig. 3 shows a schematic diagram of the platform used for the machining experiments. Units of the system included a control system, hydraulic system, and power system. In other words, the servo system controlled precise movement along the $\mathrm{x}-, \mathrm{y}-$, and $\mathrm{z}$-axes as well as the direction of rotation of the electrode. The three-unit four-passage solenoid valve was used to change the mode of internal or external flushing. The power system provided DC voltage and pulse voltage. A reasonable combination of the power system, internal or external flushing, and the clockwise or anticlockwise rotation of the electrode could be used to form high-quality holes. 


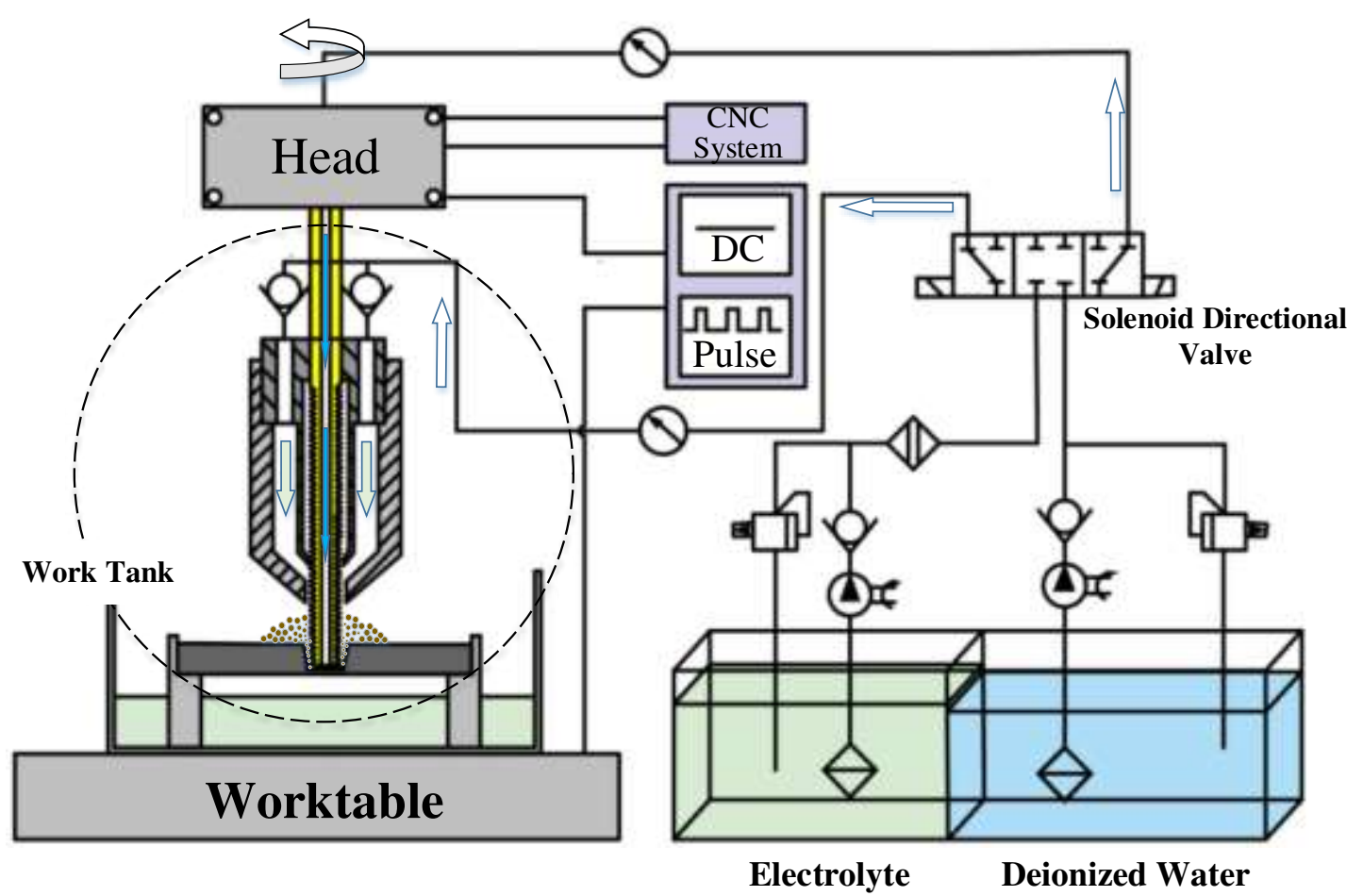

Fig. 3 Experimental apparatus of a combination of high-speed EDM and ECM using a helical tube electrode with matched internal and external flushing

\subsection{Materials}

The experimental material was a 2-mm-thick cobalt-based superalloy, and its chemical composition is shown in Table 2. The tube tool electrode was made of brass, and a helical structure with a diameter of $1 \mathrm{~mm}$ and an angle of $60^{\circ}$ was formed on its outer surface, as shown in Fig. 4.

\section{Table 2}

Elemental components of cobalt-based superalloy (DZ40M)

\begin{tabular}{lllllllllll}
\hline Elements & $\mathrm{C}$ & $\mathrm{Cr}$ & $\mathrm{Ni}$ & $\mathrm{W}$ & $\mathrm{Mo}$ & $\mathrm{Al}$ & $\mathrm{Ti}$ & $\mathrm{Ta}$ & $\mathrm{Zr}$ & $\mathrm{Co}$ \\
\hline $\mathrm{Wt} \%$ & $0.45 \sim$ & $24.5 \sim$ & $9.50 \sim$ & $7.0 \sim$ & $0.1 \sim$ & $0.7 \sim$ & $0.05 \sim$ & $0.1 \sim$ & $0.1 \sim$ & Remai \\
& 0.55 & 26.5 & 11.5 & 8.0 & 0.5 & 1.2 & 0.30 & 0.5 & 0.3 & $\mathrm{n}$ \\
& & & & & & & & & & \\
\hline
\end{tabular}



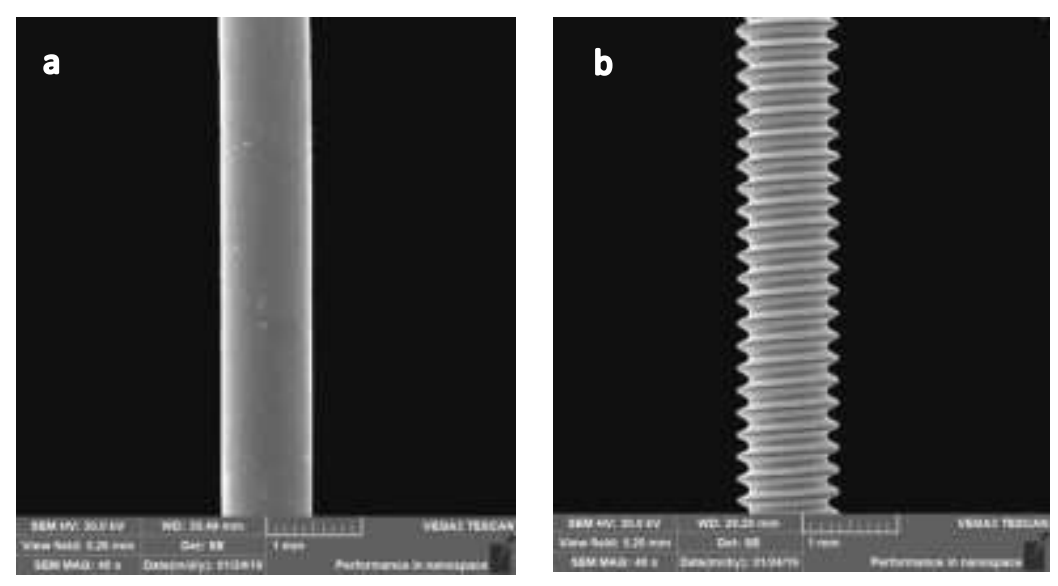

Fig. 4 SEM images of the tube electrode fabricated with a smooth surface and a helical structure

3.4 Design and conditions for simulation and experiments

The mechanism of the combination of high-speed EDM and ECM with internal and external flushing was confirmed, and the consequent improvement in machining performance was verified by comparative simulations and experiments involving a cylinder tube electrode and a helical electrode. The parameters used for comparison are shown in Table 3. To further improve the hole-forming performance of high-speed EDM and ECM, the key parameters influencing the flushing pressure and rotational speed of the electrode were optimized by experiments. The variables for them are shown in Table 4. 


\section{Table 3}

Parameters used in comparative simulations and experiments

\begin{tabular}{|c|c|c|}
\hline & Parameter & Values \\
\hline & Tube electrode diameter & $1 \mathrm{~mm}$ \\
\hline & Working solution & Deionized water \\
\hline high- & Pulse width & $12 \mu \mathrm{s}$ \\
\hline speed & Pulse interval & $12 \mu \mathrm{s}$ \\
\hline \multirow[t]{6}{*}{ EDM } & Peak current & $14 \mathrm{~A}$ \\
\hline & Rotational speed of tool electrode & $300 \mathrm{rpm}$ \\
\hline & External flushing pressure & $4 \mathrm{MPa}$ \\
\hline & Tube electrode diameter & $1 \mathrm{~mm}$ \\
\hline & Working solution & $\mathrm{NaNO}_{3}$ \\
\hline & Electrolyte concentration & $4 \mathrm{~g} / \mathrm{L}$ \\
\hline \multirow[t]{4}{*}{$\mathrm{ECM}$} & ECM voltage & $45 \mathrm{~V}$ \\
\hline & ECM time & $20 \mathrm{~s}$ \\
\hline & Rotational speed of tool electrode & $300 \mathrm{rpm}$ \\
\hline & External flushing pressure & $4 \mathrm{MPa}$ \\
\hline
\end{tabular}




\section{Table 4}

Variables used in parameter optimization experiment

\begin{tabular}{lll}
\hline & Parameter & Values \\
high- & Rotational speed of tool electrode & $60 \mathrm{rpm}, 160 \mathrm{rpm}, 260 \mathrm{rpm}, 360$ \\
speed & $\mathrm{rpm}, 460 \mathrm{rpm}$ \\
EDM & Internal flushing pressure & $1 \mathrm{MPa}, 3 \mathrm{MPa}, 5 \mathrm{MPa}, 7 \mathrm{MPa}, 9$ \\
& & $\mathrm{MPa}$ \\
\hline & Rotational speed of tool electrode & $60 \mathrm{rpm}, 160 \mathrm{rpm}, 260 \mathrm{rpm}, 360 \mathrm{rpm}$, \\
ECM & & $460 \mathrm{rpm}$ \\
& External flushing pressure & $1 \mathrm{MPa}, 2 \mathrm{MPa}, 3 \mathrm{MPa}, 4 \mathrm{MPa}, 5$ \\
& & $\mathrm{MPa}$ \\
\hline
\end{tabular}

4 Verifying the combination of high-speed EDM and ECM with internal and external flushing

\subsection{Verifying improvement in flow field and machining performance in high-speed EDM}

Fig. 5 shows a cloud diagram of the axial velocity during high-speed EDM using the cylindrical tube electrode and the helical tube electrode, separately. The maximum axial velocity of the helical tube electrode was $14.29 \mathrm{~m} / \mathrm{s}$ while that of the cylindrical tube electrode was $11.97 \mathrm{~m} / \mathrm{s}$. The rotation of the cylindrical electrode caused only the circular motion of the working fluid while that of the electrode with the helical structure caused circular as well as axial motion. The rotation of the helical tube electrode led to the axial movement of the debris in the narrow gap, which followed the same principle as that of twist drilling. When the clockwise rotation of the electrode was controlled, the helical surface generated an upward axial force on the debris. Under the combined 
action of the internal flushing mode and electrode rotation, the debris was rapidly removed from the processing area. In addition, the vector and streamline diagrams on the right of Figs. 5(a) and (b) show that the flow field of the gap in the cylindrical electrode was laminar and that in the helical electrode was turbulent. The working fluid in the helical groove formed a vortex that prevent the accumulation and deposition of debris. By observing velocity at the bottom of the blind hole, we found that the corner velocity of the helical electrode was $9.44 \mathrm{~m} / \mathrm{s}$ and that of the cylindrical electrode was $7.35 \mathrm{~m} / \mathrm{s}$. The corner was most likely to cause debris to deposit in narrow gaps when the cylindrical electrode was used. Fig. 5 (c) shows the velocity of gap flow in the direction of the depth of the blind hole. It shows that when the working fluid passed through the helical groove, its speed increased, but decreased as it passed the top surface of the thread. From the bottom surface of the blind hole to the entrance, the flow rate of the working fluid showed a downward trend. The flow velocity of the gap in the helical structure was significantly higher than that in the cylindrical structure. Therefore, using the helical tube electrode can help remove more debris than the cylindrical tube electrode. 

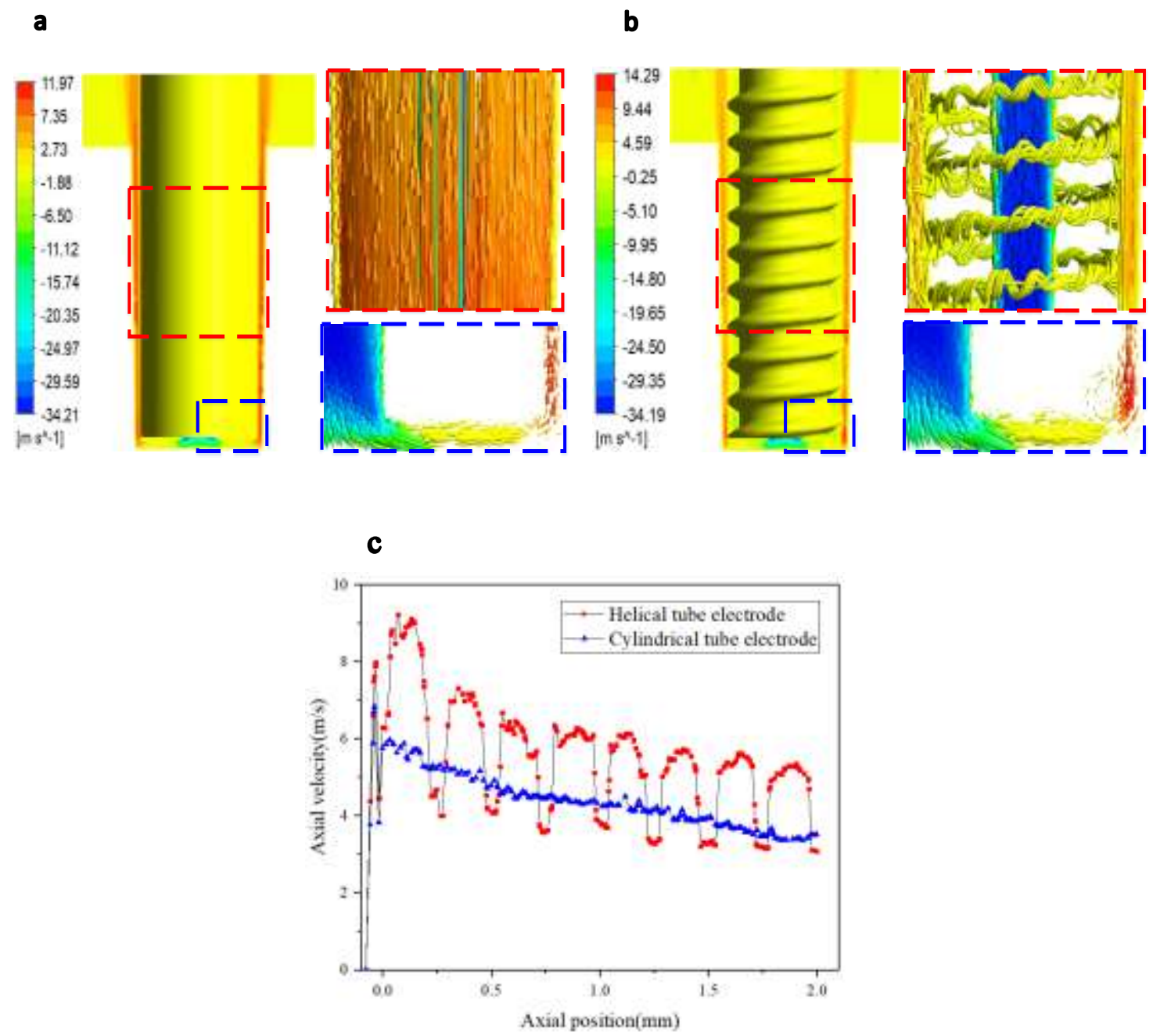

Fig. 5 Contours of axial velocity: (a) cylindrical tube electrode; (b) helical tube electrode; (c) the velocity of the working fluid along the depth direction

Figs. 6 (a) and (b) show the distributions of interstitial particles in the cylindrical and the helical tube electrodes. Fig. 6(a) shows particle accumulation on the sides and bottom of the hole, with a maximum particle concentration of $2.74 \mathrm{~kg} / \mathrm{m}^{3}$. Fig. $6(\mathrm{~b})$ shows only a small number of particles in the helical groove, with a maximum local particle concentration of $2.63 \mathrm{~kg} / \mathrm{m}^{3}$. Because the rotational movement of the cylindrical tube electrode struggled to change fluid flow along the side gap, the particle distribution was uneven in the laminar state, and material accumulated to block the side gap. By contrast, the helical flow channel enlarged the side gap, and the continual change in the side wall of the helical region increased disturbance in the flow field of 
the gap that helped avoid the accumulation of particles in the gap. The rotation of the helical electrode produced an axial compression force on the medium in the groove, and the particles thus quickly discharged under the action of extrusion and fluid viscosity. Therefore, particle concentration helical tube electrode and their area of distribution were small.

The particle concentrations in the helical groove and side gaps were examined, and are shown in Figs. 6(c) and (d). Fig. 6(c) shows that the particle concentration first decreased and then increased from the surface of the helical electrode to the wall of the hole, and the maximum particle concentration in the helical groove was $0.2 \mathrm{~kg} / \mathrm{m}^{3}$. The particle concentration of the cylindrical tube electrode gradually increased, and the maximum appeared in the hole in the wall, $0.65 \mathrm{~kg} / \mathrm{m}^{3}$. In Fig. $6(\mathrm{~d})$, the particle concentration of the helical tube electrode is significantly lower than that of the cylindrical tube electrode. The higher particle concentration in the groove of the helical electrode means that the helical structure can reduce the number of particles in the side gap. The vortex generated in the groove increases the local energy loss of the fluid, and a small number of particles are deposited in the concave. Therefore, the use of the helical tube electrode during high-speed EDM drilling can ensure that the product is removed in time and guarantees the processing quality of the hole. 

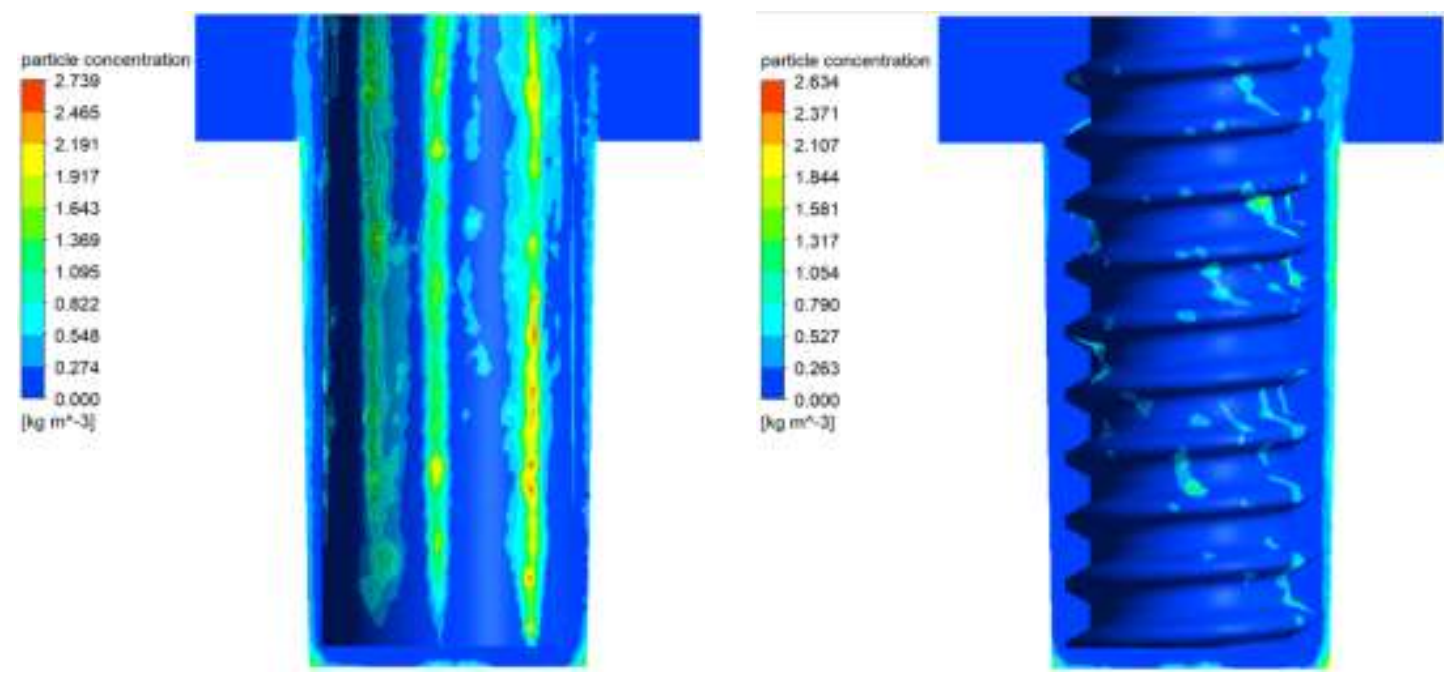

\section{c}

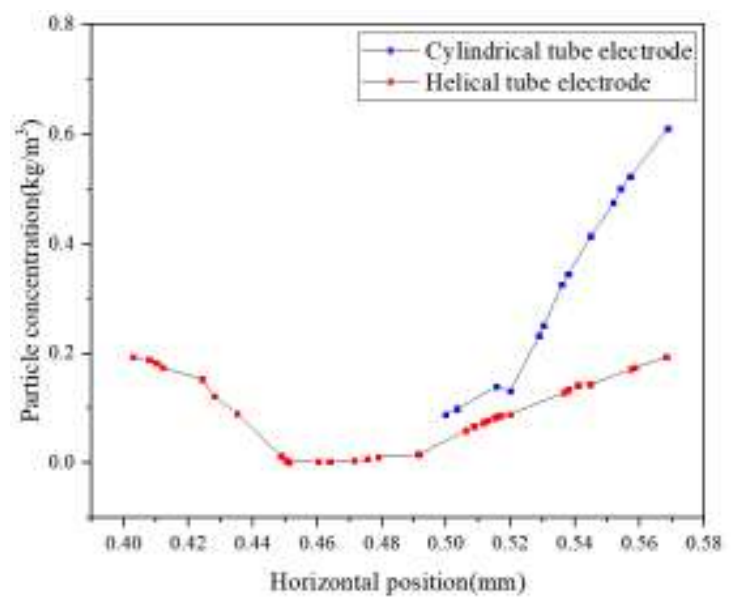

d

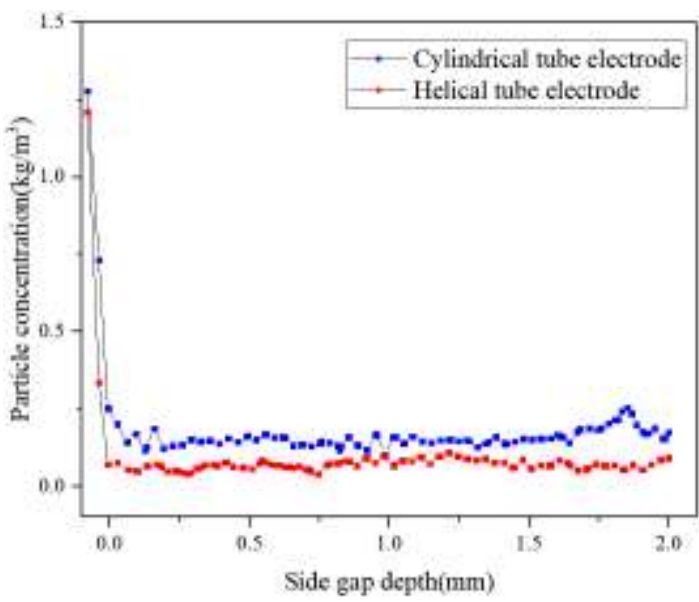

Fig. 6 Cloud diagram of particle concentrations in the gap in case of (a) cylindrical tube electrode; and (b) helical tube electrode. Distribution of particle concentration in the helical groove and the side gap (c) and (d)

The exit diameters, rates of material removal, and tapers of the hole as machined by the helical electrode and cylindrical electrode were compared as shown in Fig. 7. The entrance and exit morphologies of the hole are shown in Fig. 8. The exit diameter of a perforation in case of the cylindrical electrode was $0.94 \mathrm{~mm}$, and the edges of the aperture were rough. The exit diameter as machined by using a helical electrode was 
$0.98 \mathrm{~mm}$, and was round and smooth. Because the rotation of the cylindrical electrode can promote only the circular motion of the debris in the gap, the debris flowed out of the processing area under the action of the internal flushing pressure. The rotation of the helical electrode subjected the debris to an axial force that generated axial movement, and it was easily expelled. Secondary discharge was reduced by increasing debris removal in the narrow gap. Therefore, the loss of the helical electrode was reduced and the outlet aperture was increased. The results of material removal rate show that the MRR, when the helical electrode was used, was higher than that when the cylindrical electrode was used. This is because the helical electrode removed debris more quickly such that this rendered the high-speed EDM process more stable, and shortened the processing time. With regard to the taper, that in case of cylindrical electrode drilling was 0.04 and in case of a helical electrode was 0.025 . Because the end-face velocity of the blind hole in case of the cylindrical electrode was low, the debris easily accumulate, and the electrode loss more mass.

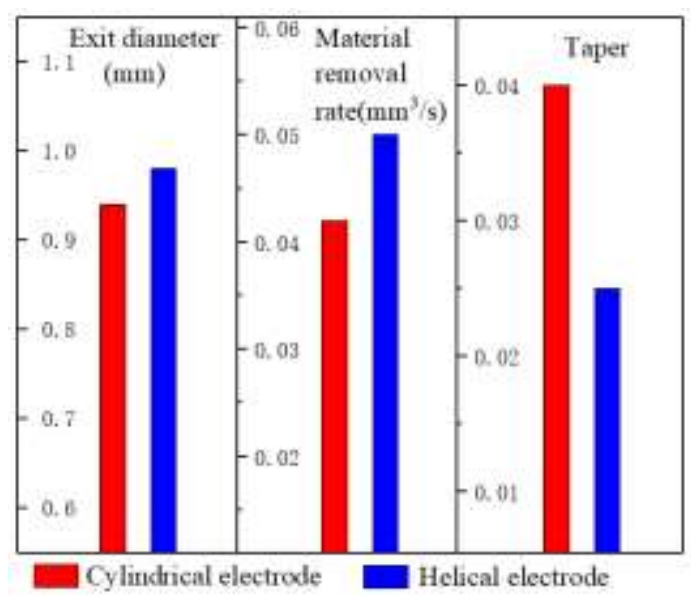

Fig. 7 Effects of electrode structure on the exit diameter, MRR, and taper of hole in case of high-speed EDM 

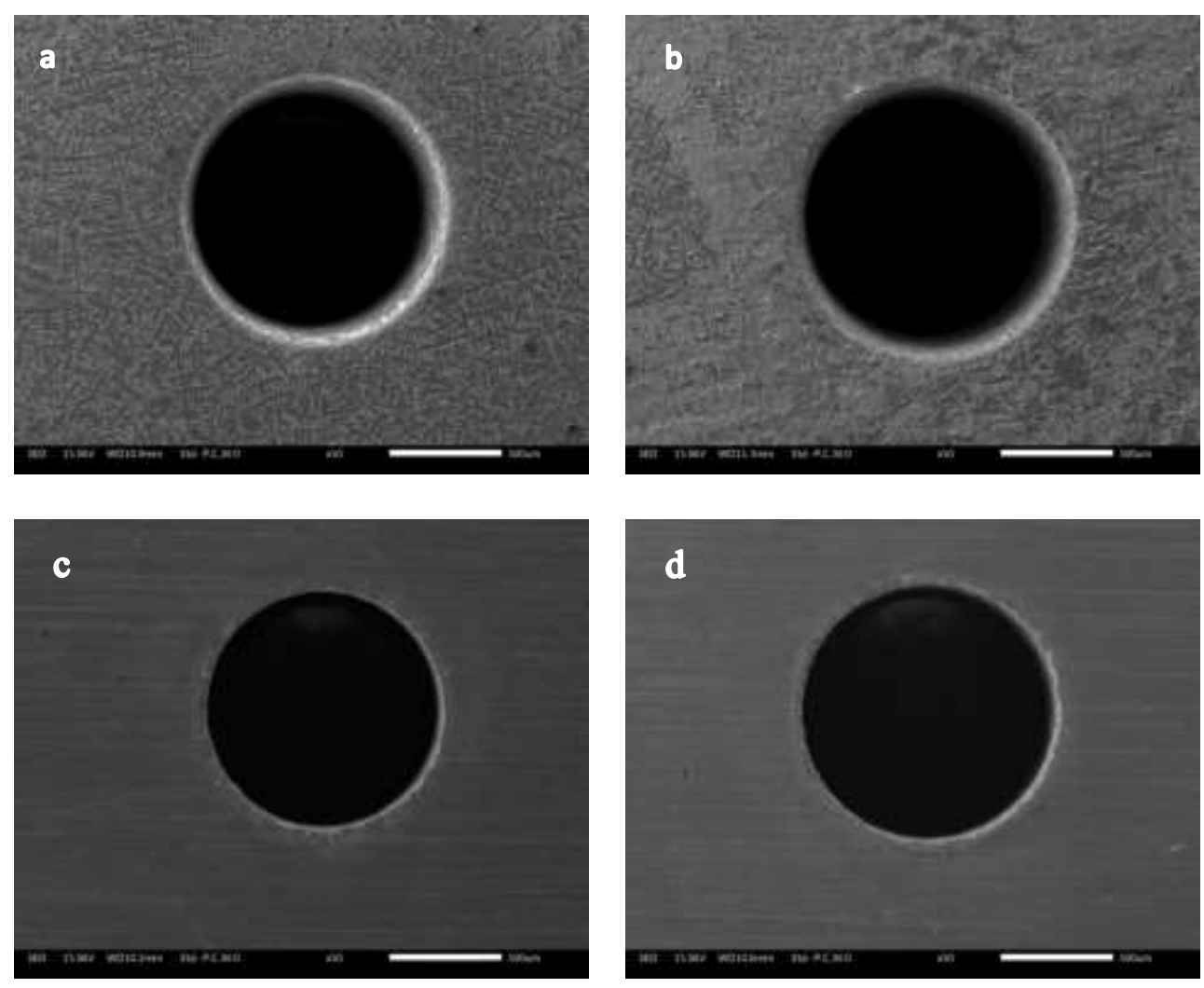

Fig. 8 Morphologies of the entrance and the exit in high-speed EDM: (a) entrance, cylindrical tube; (c) exit, cylindrical tube electrode; (b) entrance, helical tube electrode; and (d) exit, helical tube electrode

Fig. 9 shows the cross-sectional morphology of the hole of the wall subjected to electrode drilling. Fig. 9(a) shows the results, which reflect a large taper and poor exit accuracy. This is because the removal debris can rely only on the mode of flushing. When the hole is only punctured, the capability of the mode of flushing to speed-up debris removal is weakened. At this time, the debris in the gap becomes difficult to removal, because of which the quality of processing of the outlet is poor. Fig. 9(b) shows the results of drilling in case of the helical electrode. When the hole was punctured, the reverse flushing effect became weaker in the lateral gap, but the helical groove enhanced the effect of debris removal to ensure processing stability and the quality of the hole. To sum up, using a helical electrode in high-speed EDM can reduce the processing time, improving processing efficiency, reducing hole taper, and render 
the edges of the hole smoother and rounder. In high-speed EDM where internal flush mode matches with the clockwise rotation of the electrode, the helical tube electrode is more conducive to machining high-quality micro-holes.
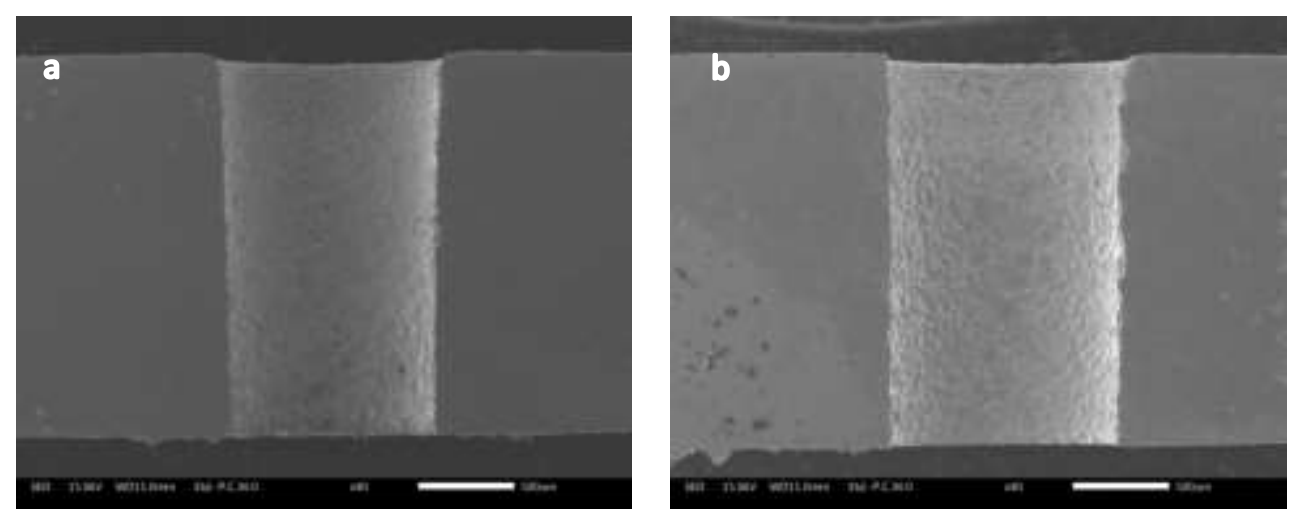

Fig. 9 The morphology of the hole in the wall in high-speed EDM: (a) cylindrical tube electrode; (b) helical tube electrode

\subsection{Verifying improvements in flow field and machining performance in ECM}

Fig. 10 shows the influence of electrode structures on the distribution of gap flow during the ECM process. Fig. 10(a) shows that the flow of working fluid obeyed laminar flow for the cylinder tube electrode and vortical flow for the helical tube electrode as shown in Fig. 10(b). Measuring the gap flow velocity in the axial direction revealed that it was significantly higher for the helical tube electrode, as shown in Fig. 10(c). The difference between the flow velocities reached the maximum at the helical groove. When the electrolyte flowed through the helical groove, a vortex was generated in it. Under the action of the vortex, the products of the electrolyte were difficult to accumulate in the processing area. When the electrode rotated counterclockwise, the upper surface of the helical groove exerted an axial downward force on the product, thus accelerating the removal rate of hydroxide products of ECM. In addition, due to the processing of the narrow gap, the helical electrode and the narrow gap formed a screw pump structure. As the helical electrode rotated, the working fluid at the inlet was sucked into the processing zone, thereby increasing the axial speed of the working fluid. 
It was then difficult for the cylindrical electrode to provide axial velocity owing to its smooth surface. When using cylindrical electrodes, the electrolyte in the gap can flow only to the outlet by gravity and the initial pressure of the flushing fluid. Therefore, the gap velocity of cylindrical electrode was lower than that of the helical electrode. To sum up, the rotation of the cylindrical electrode can cause the electrolyte to move only in a circle, and electrolyte flow in the gap can rely only on gravity and the pressure at the entrance. The rotational motion of the helical electrode can not only increase flow in the gap, but can also accelerate flow field disturbance. This causes the medium to generate axial velocity, which accelerates the flow velocity of the gap in turn. 

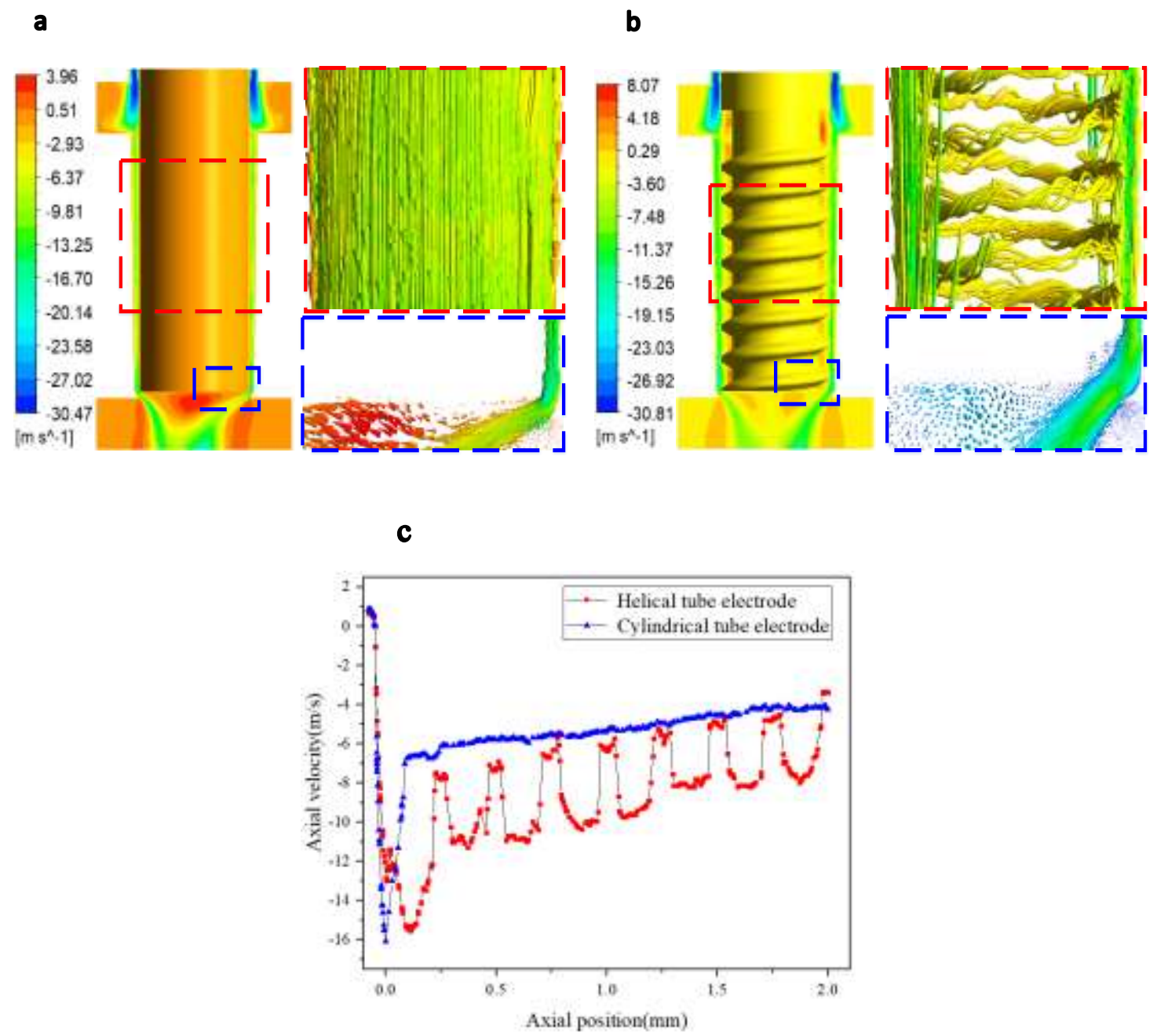

Fig. 10 Contours of axial velocity in the breakthrough hole stage: (a) cylindrical tube electrode; (b) helical tube electrode; (c) velocity of working fluid in the direction of hole depth

Fig. 11 shows the particle concentration of the cylindrical tube electrode and the helical tube electrode during ECM. The maximum particle concentration in Fig. 12(a) is $11.885 \mathrm{~kg} / \mathrm{m}^{3}$. The partially enlarged view shows that the particle concentration at the wall of the hole was relatively high, and that it was difficult to remove the electrolytic products in the gap using only the external flushing method. In Fig. 12(b), although the maximum particle concentration was $13.833 \mathrm{~kg} / \mathrm{m}^{3}$, greater than that of the cylindrical electrode, this appeared only at the outlet. Particles in the narrow gap on the side were removed. The counterclockwise rotation of the helical structure had a downward 
squeezing effect on the working fluid in the gap. The medium flowed rapidly under the initial flow pressure, gravity, and the compression of the helical groove, and the electrolytic product was quickly removed from the gap.

Figs. 11(c) and (d) show curves of the particle concentrations in the side gap and groove along the radial direction in ECM. The "0.0" position in Fig. 11(c) indicates the exit and the "2.0" position indicates the entrance. Fig. 11(c) shows that the particle concentration on the side gap of the helical tube electrode was lower than that for the cylindrical tube electrode, and gradually increased from the inlet to the outlet. The disturbance capability of the helical groove and the increasing the side gap sped-up product removal and improved the environment of the side gap. Fig. 11(d) shows a comparison of the concentration of radial particles between the surface of the electrode and the wall in the hole, where the former was lower than the latter. Therefore, regardless of whether radial comparison or axial comparison was used, the helical tube electrode was found to be conducive to accelerating the regeneration of the side gap of the electrolyte as well as the product removal. 
a

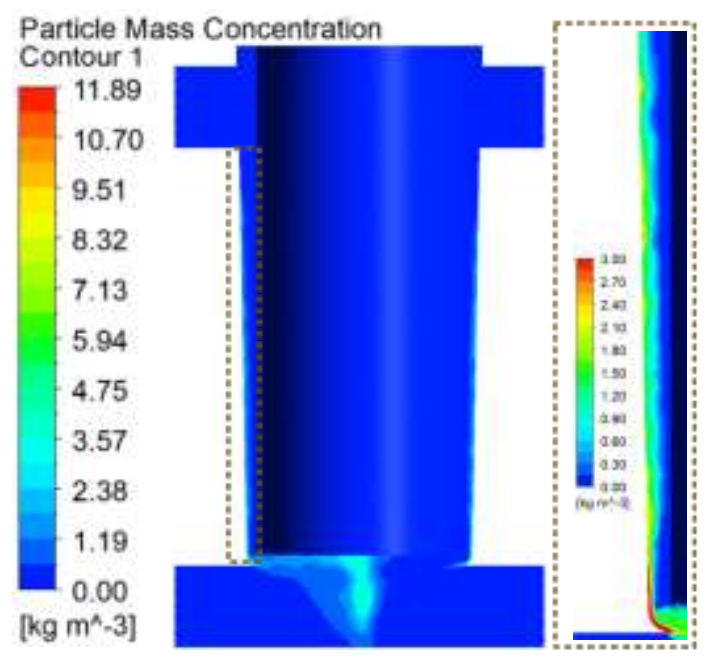

C

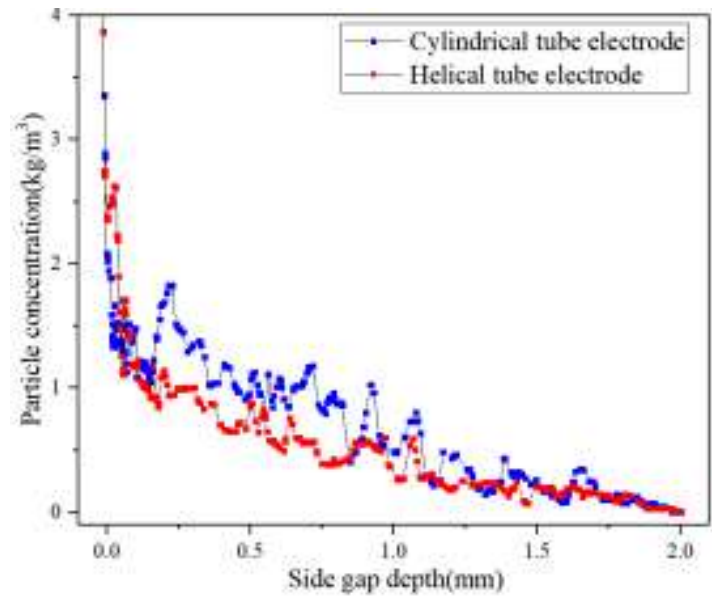

b

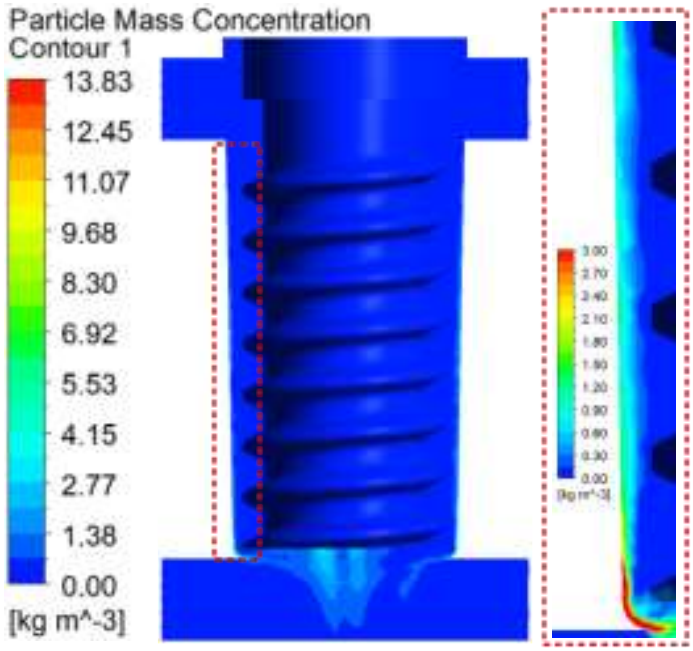

d

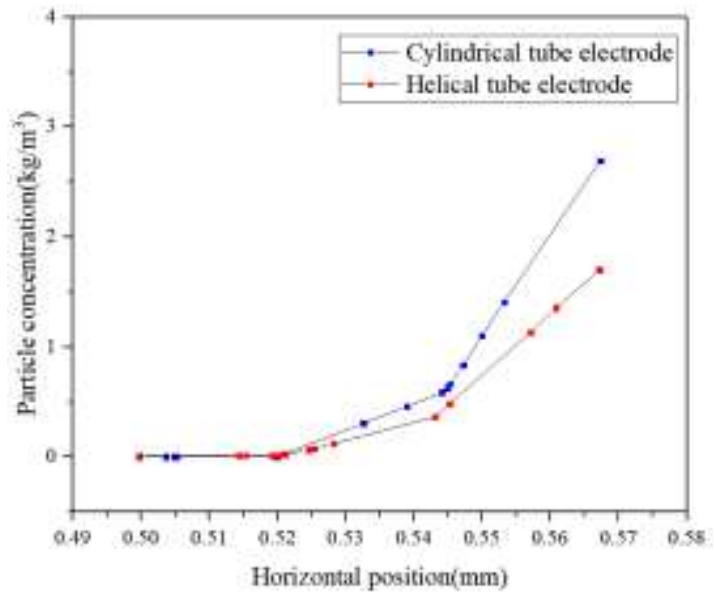

Fig. 11 Cloud diagram of particle concentration in ECM, using: (a) cylindrical tube electrode; and (b) helical tube electrode. Particle concentration distribution in the helical groove and the side gap in ECM, (c) and (d)

As shown in the Fig. 12, ECM experiments with external flushing were carried out to compare the effects of electrode structures on machining performance, including in terms of the hole exit, material removal rate, and taper. The exit diameter of the cylindrical electrode was set to $1.07 \mathrm{~mm}$ and that of the helical electrode to $1.17 \mathrm{~mm}$. The rotation of the cylindrical electrode generated only circumferential force on the electrolyte, and it was thus difficult to transport the electrolyte to the electrolytic area, 
especially the exit position, using only external flushing within the narrow gap. This made it difficult to remove hydrogen and oxygen products, and to update the electrolyte, which in turn made it challenging to realize the electrolytic reaming of the cylindrical electrode. This rendered the outlet of the hole small and the taper large. For the material removal rate, the electrolyte in the gap is updated slowly, and the electrolysis effect is poor. However, the counterclockwise rotation of the helical electrode generated axial thrust on the electrolyte in the gap, as in external flushing, and finally sped-up its renewal. Therefore, the rate of material removal owing to electrochemical machining in case of a helical electrode was high. The taper of the cylindrical electrode electrolytic ream was 0.01 while that of helical electrode was 0.005 . Therefore, under the same electrochemical processing conditions, the rotation of the helical electrode sped-up the electrolyte to a greater extent, and the effect of electrolysis of the outlet of the hole was significant; thus, the taper was small and accuracy was high.

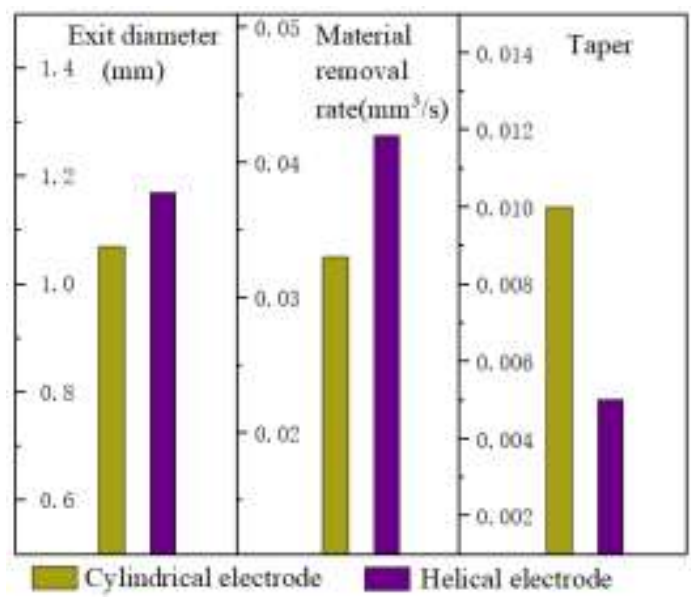

Fig. 12 Effects of the electrode structure on the diameter of the exit, the MRR, and the taper in ECM

Fig. 13 shows the morphologies of the entrance and the exit of holes drilled using 
different electrodes in the ECM stage. It shows that the roundness of the reaming of the cylindrical electrode was poorer than that of the helical electrode because the electrolyte struggled to flow into the narrow machining gap in the former case, resulting in a lack of liquid in the gap. The electrolytic products thus easily accumulated, affecting the stability of rotation of the cylindrical electrode. Therefore, the effect of the electrochemical reaction and hole roundness were poor. In case of the helical structure, the narrow gap was approximated as a screw pump structure. When the electrode rotated, the electrolyte at its entrance was sucked into the processing zone. Meanwhile, the helical surface sped-up the downward flow of the electrolytic products and avoided product accumulation. Therefore, the electrochemical machining in the gap was more uniform, the stability of the electrode was guaranteed, and the inlet and outlet roundness values of hole were improved. 

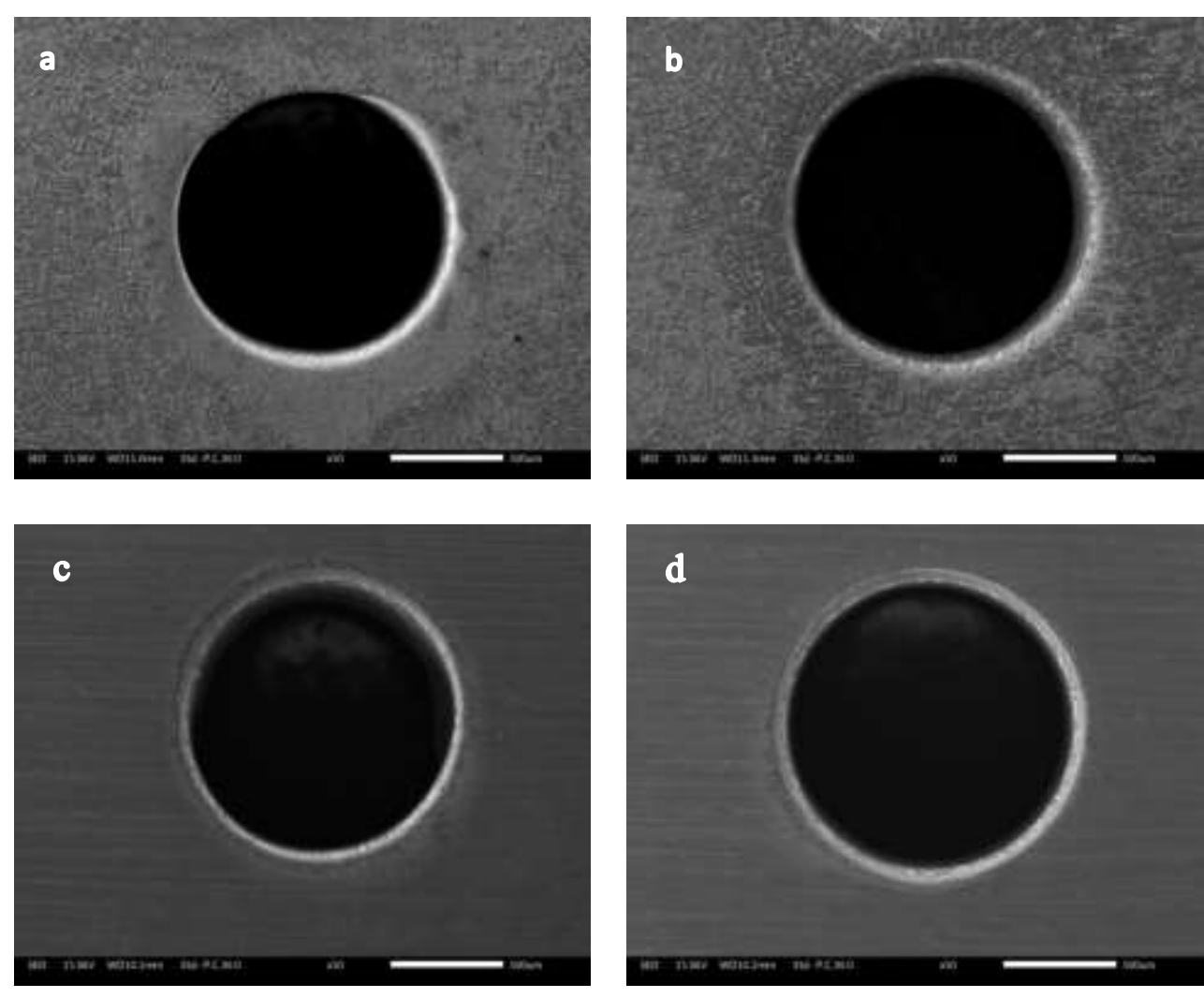

Fig. 13 Influence of electrode structure on the roundness of the entrance and exit in

ECM: (a) entrance, cylindrical electrode; (b) entrance, helical electrode; (c) exit, cylindrical electrode; (d) exit, helical electrode

As can be seen from the Fig. 14(a), some partial surface of the hole wall still exist the thermal corrosion layer when using cylindrical electrode in electrochemical machining. Local magnification also clearly showed defects in the wall, such as microcracks, spherical particles, and rough surfaces. Because the electrolyte could not flow quickly into extremely narrow gaps, the hydroxide and bubbles could not be removed, and the retention of the product led to unstable electrochemical machining that resulted in secondary discharge in the holes in the wall. The holes on the wall of the helical electrode were smooth without prominent defects. Fig. 14(b) shows that the helical electrode rotated counterclockwise to create an axial velocity against the hydroxide. 
The motion of the electrode increased disturbance which prevented the product from accumulating. The rate of flow of the medium in the gap increased, the effect of the electrolytic improved, and defects due to holes in the wall decreased.
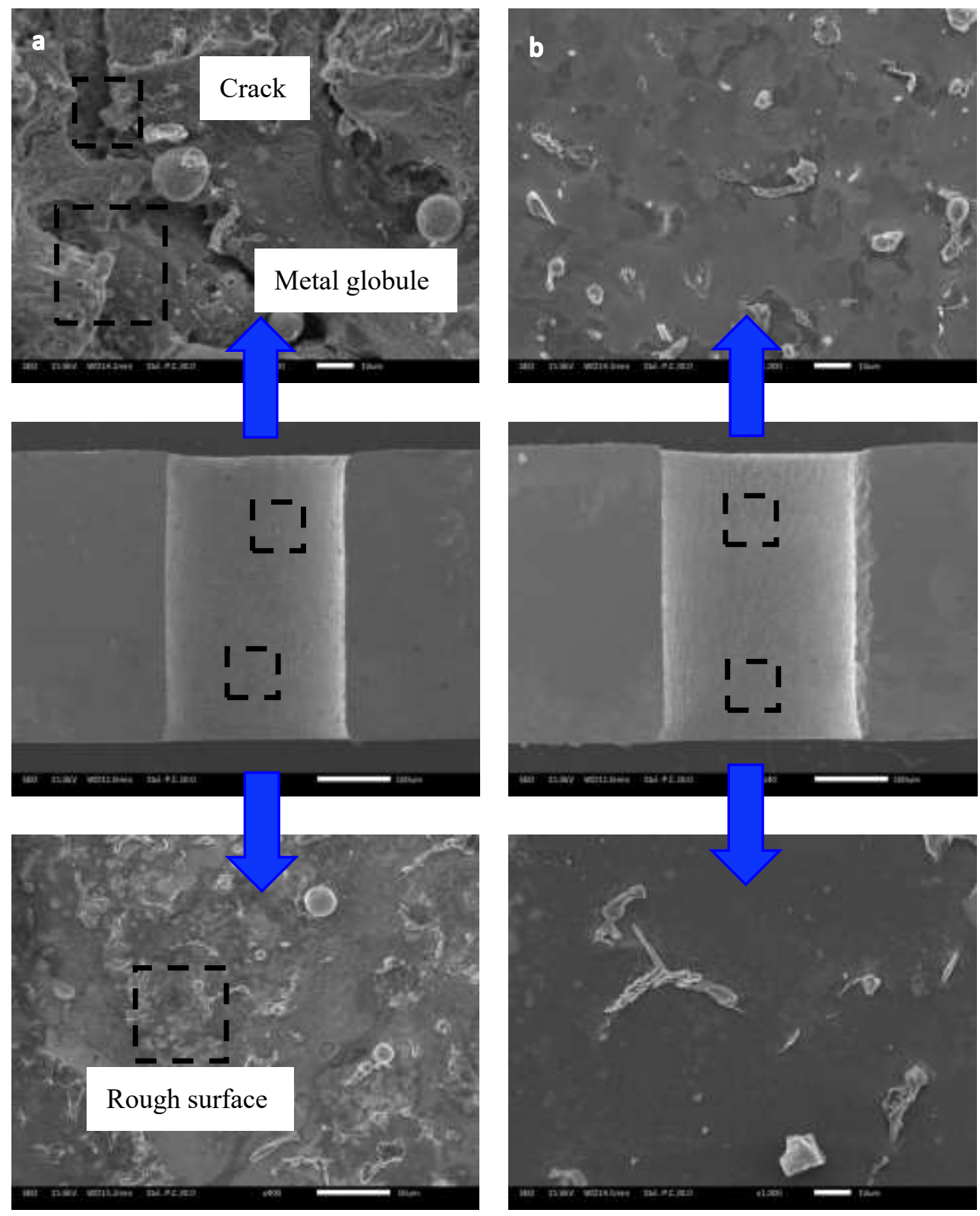

Fig. 14 The morphology of holes in the wall after combined machining: (a) cylindrical electrode; (b) helical electrode

To sum up, electrochemical reaming with a helical electrode can significantly improve the accuracies of the entrance and exit, and reduce the taper. The holes in the wall can be smoothened without defects. In electrochemical machining with electrodes 
rotating counterclockwise matched by external flushing, the effect of machining using the helical electrode is better than that using the cylindrical electrode.

\section{Performance analysis of combination of high-speed EDM and ECM using a helical tube electrode with matched internal and external flushing}

The above-mentioned flow field simulation and experiment showed that the helical tube electrode can be used to improve hole-forming performance compared with the cylindrical tube electrode. To further improve high-speed EDM and ECM processing in terms of the holes formed, we examined the key parameters of flushing pressure and the speed of the electrode.

\subsection{Influence of internal flushing pressure on high-speed EDM}

The effects of different internal flushing pressures on machining performance were studied, as shown in Fig. 15. The diameter of the exit of the hole gradually decreased with increasing flushing pressure. The minimum diameter of the exit was $0.99 \mathrm{~mm}$, and the exit morphologies of the hole under different flushing pressures are shown in Fig. 16. The exits became rounder as the internal flushing pressure increased. This indicates that matching the internal flushing with the clockwise rotation of the helical electrode can accelerate the flow rate of the working fluid in the narrow gap. Thus, the debris was quickly removed, which can improve machining accuracy. The rate of material removal increased as the pressure increased. The maximum material removal rate was $0.122 \mathrm{~mm}^{3} / \mathrm{s}$. Because the internal flushing pressure accelerated the velocity of the working fluid and improved the performance of high-speed EDM, the material removal rate was improved. The taper of the hole decreased as the internal flushing pressure increased. The minimum taper was 0.014 . Fig. 17 shows that the trend of the taper was consistent with the curve in Fig. 15(b). The machining products reduction is beneficial to reduce the electrode wear and ensure the high shape precision 
of tube electrode, and the taper of the hole was small. Besides, the internal flushing pressure increased, high-speed EDM became stable, and machining efficiency was high, thus the machining time difference between the entrance and the exit was small, and the hole had a smaller taper. Fig. 17 also shows the morphology of the surface of the wall of holes under different internal flushing pressures. As the internal flushing pressure increased, the surface quality of the holes in the wall gradually increased. Under a low flushing pressure, the working fluid flowed in the machining gap from the inner hole of the tube electrode and hit the bottom of the blind hole, because of which velocity decreased significantly. Therefore, it became difficult for the debris in the narrow gap to be quickly removed, especially at the exit. This rendered high-speed EDM unstable, and even resulted in secondary discharge that increased surface roughness of the wall. In summary, increasing the internal flushing pressure can increased the speed of debris removal in the gap. The processing time of high-speed EDM was shorter and the diameter of exit was smaller. The MRR was higher, as was the precision of the hole. It was more circular in shape. The optimal internal flushing parameter was $9 \mathrm{MPa}$. 
a

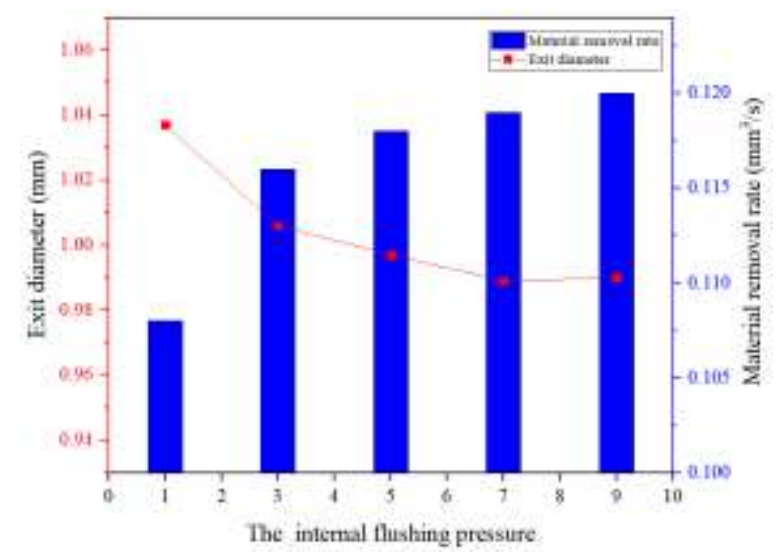

b

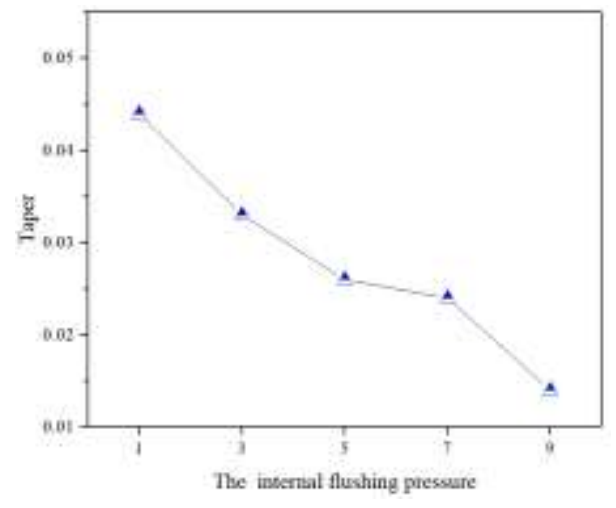

Fig. 15 Influence of different internal flushing pressures on machining performance in high-speed EDM: (a) diameter of the exit and the MRR; (b) taper
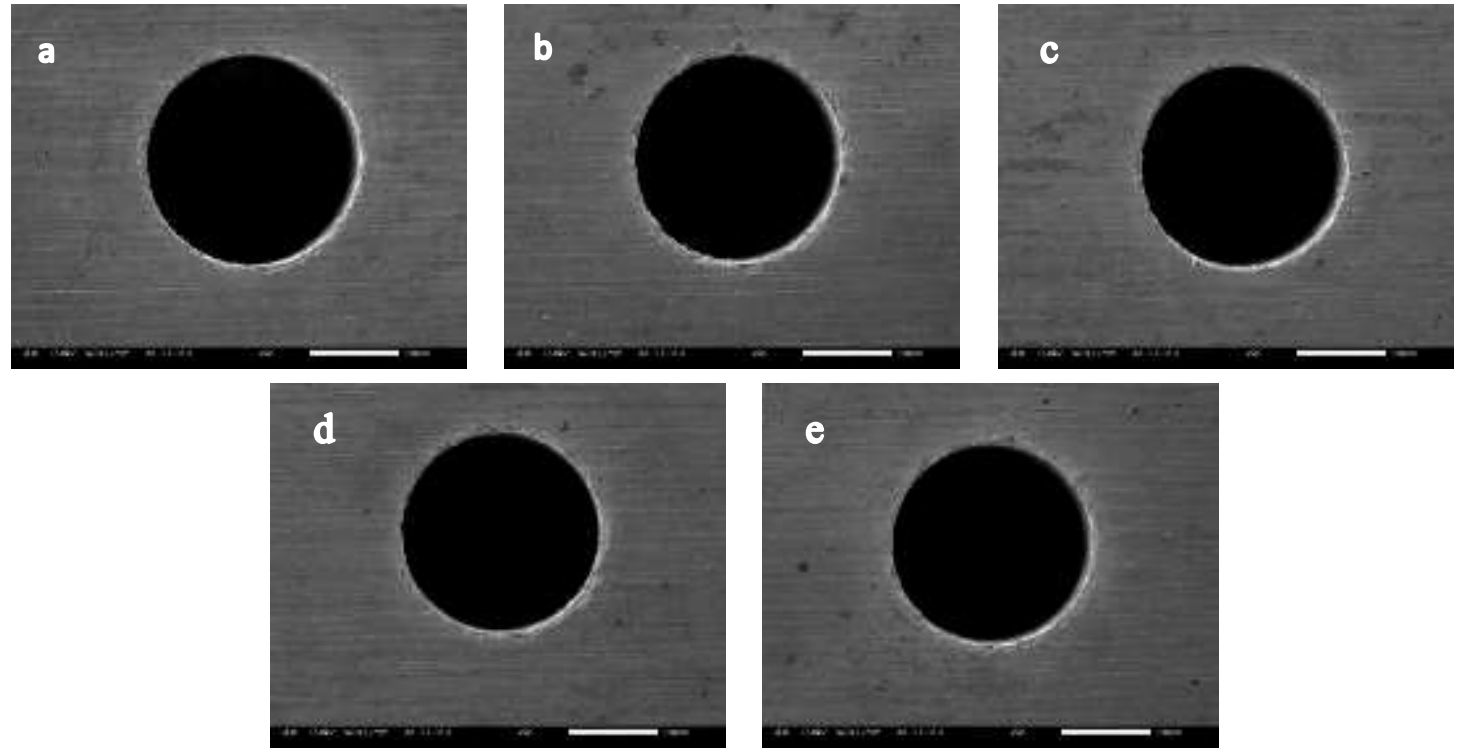

Fig. 16 The morphologies of the exit in high-speed EDM: (a) $1 \mathrm{MPa}$; (b) $3 \mathrm{MPa}$; (c) 5 $\mathrm{MPa}$; (d) $7 \mathrm{MPa}$; (e) $9 \mathrm{MPa}$ 

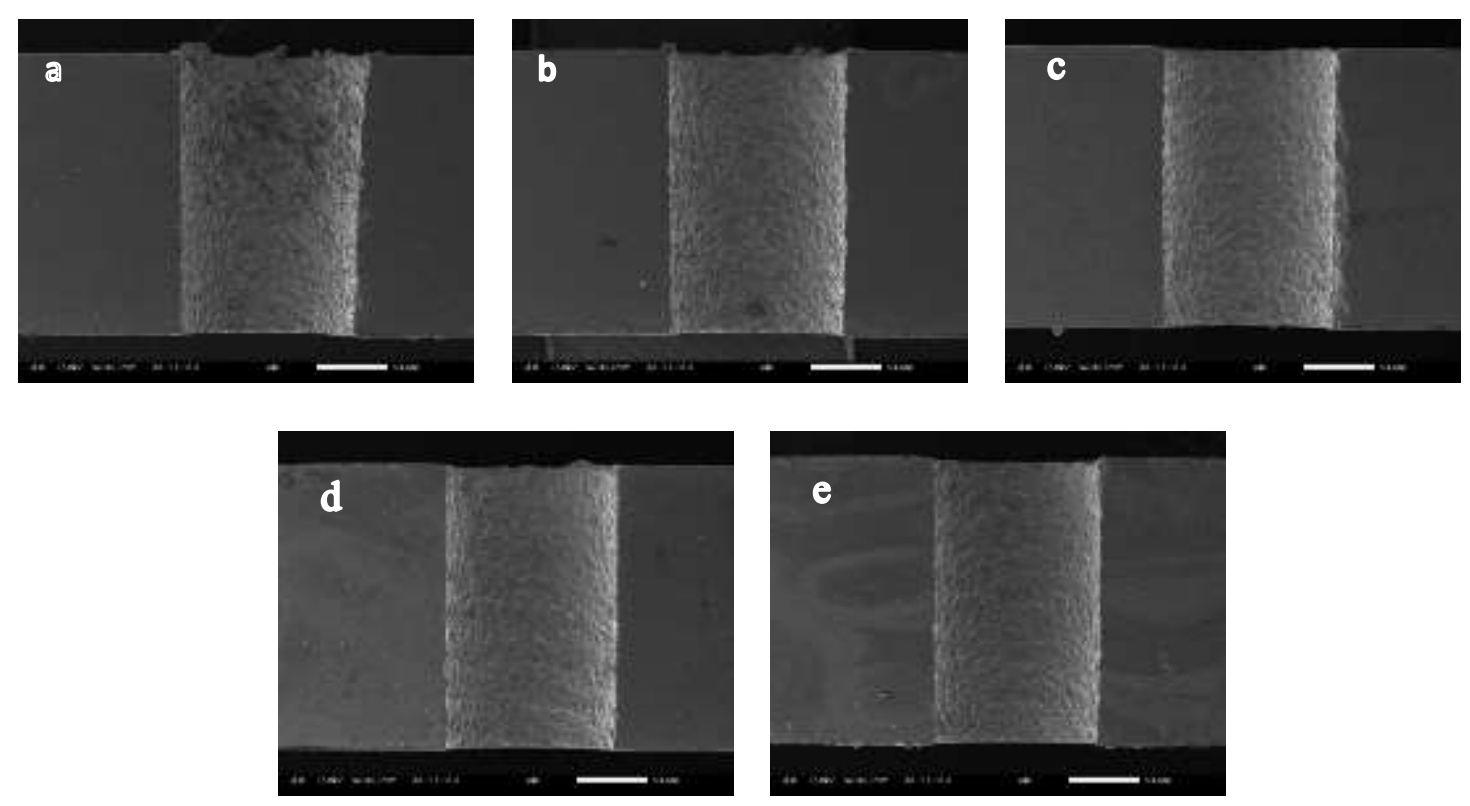

Fig. 17 The morphologies of the holes in the wall in high-speed EDM: (a) $1 \mathrm{MPa}$; (b) $3 \mathrm{MPa}$; (c) $5 \mathrm{MPa}$; (d) $7 \mathrm{MPa}$; (e) $9 \mathrm{MPa}$

\subsection{Influence of rotating speed of tool electrode on high-speed EDM}

The effects of the rotating speed of the electrode on the exit aperture, taper, and rate of material removal rate are shown in Fig.18. The exit aperture decreased as the rotating speed increased, and the minimum aperture was $0.97 \mathrm{~mm}$. The SEM morphology of the exit is shown in Fig. 19. An increasing in the speed of rotation smoothened the edge of the hole, reduced defects, and rendered it rounder. The MRR increased with the speed of rotation, and the maximum material removal rate was 0.046 $\mathrm{mm}^{3} / \mathrm{s}$. The taper decreased as the rotating speed increased, and the minimum taper was 0.02. Fig. 20 also shows the results of the taper and the quality of the holes in the wall under different speeds of rotation of the electrode. As the rotating speed increased, the upward force of the subsurface of the helical groove on the debris increased. The debris generated an axial velocity, and the internal flushing caused the medium to flow upward in the gap such that the debris could be quickly discharged. To summarize, increasing 
the speed of rotation of the electrode can accelerate the rate of removal of the debris, thereby increasing the frequency of effective discharges, improving processing efficiency, and reducing the taper of the hole and making its orifice smoother. The optimum rotating speed for high-speed EDM was $460 \mathrm{rpm}$.

a

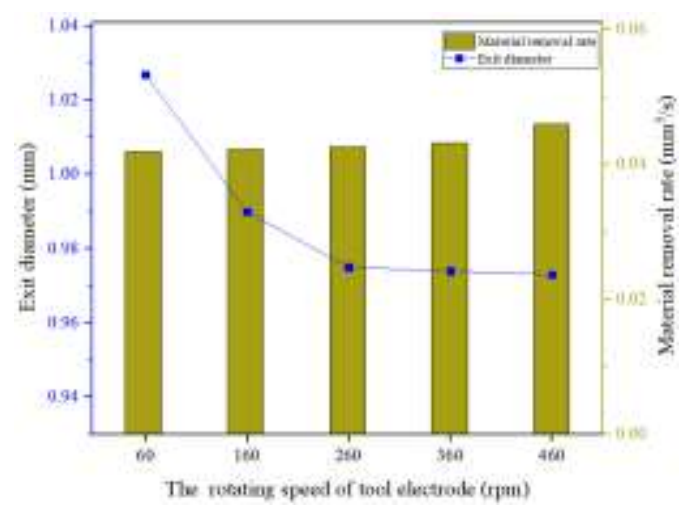

b

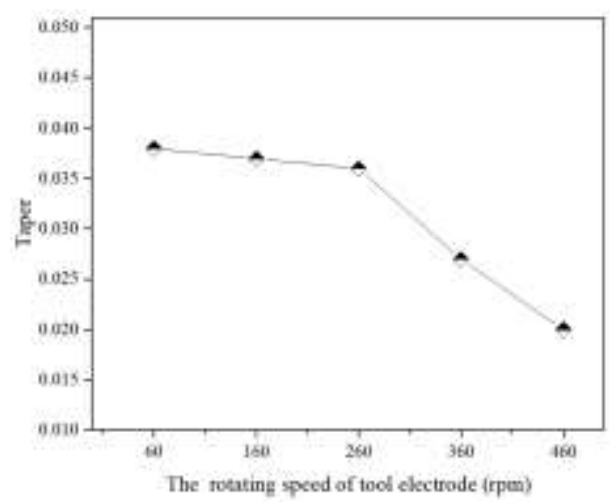

Fig. 18 Influence of different rotating speeds on machining performance in high-speed EDM: (a) diameter of exit and the MRR; (b) taper 

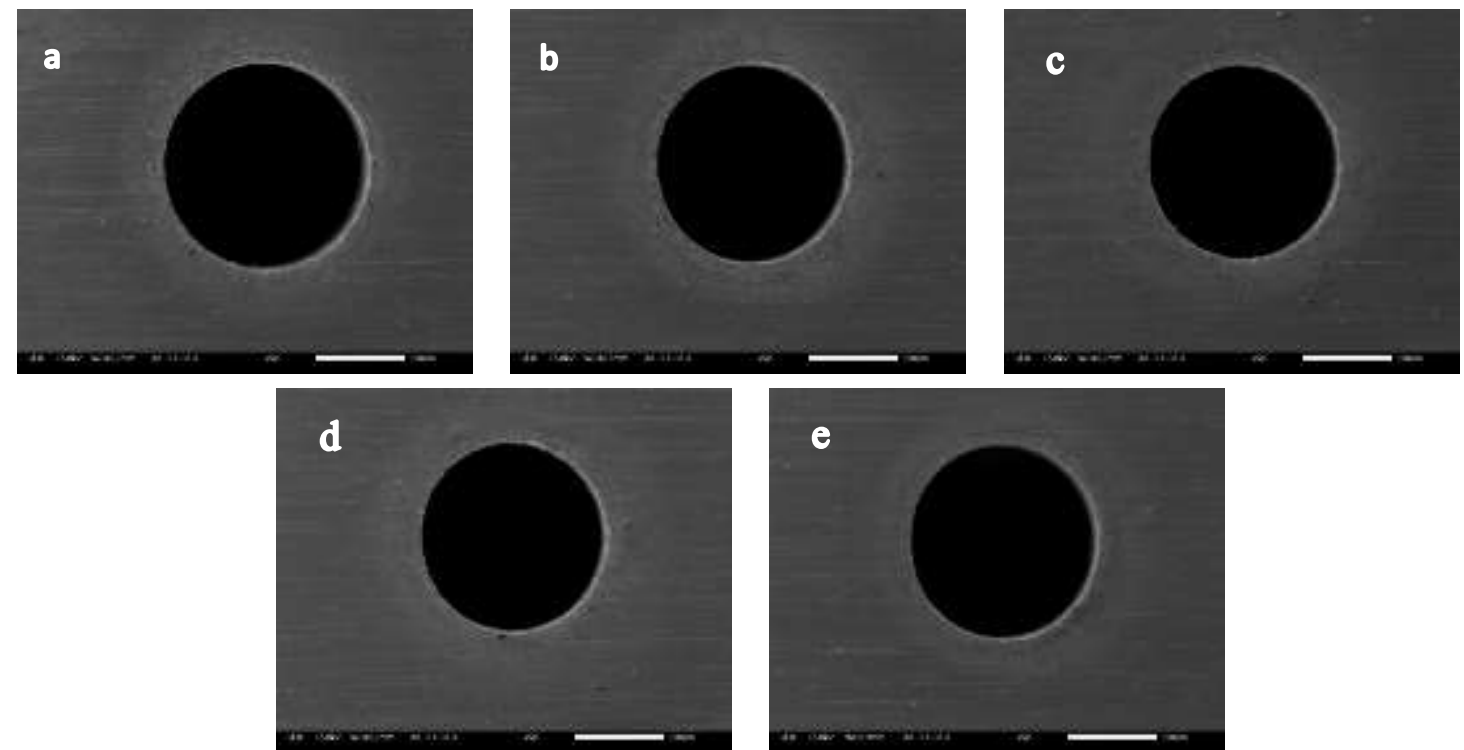

Fig. 19 The morphologies of the exit at different rotating speeds of the tool electrode in high-speed EDM: (a) $60 \mathrm{rpm}$; (b) $160 \mathrm{rpm}$; (c) $260 \mathrm{rpm}$; (d) $360 \mathrm{rpm}$; (e) $460 \mathrm{rpm}$
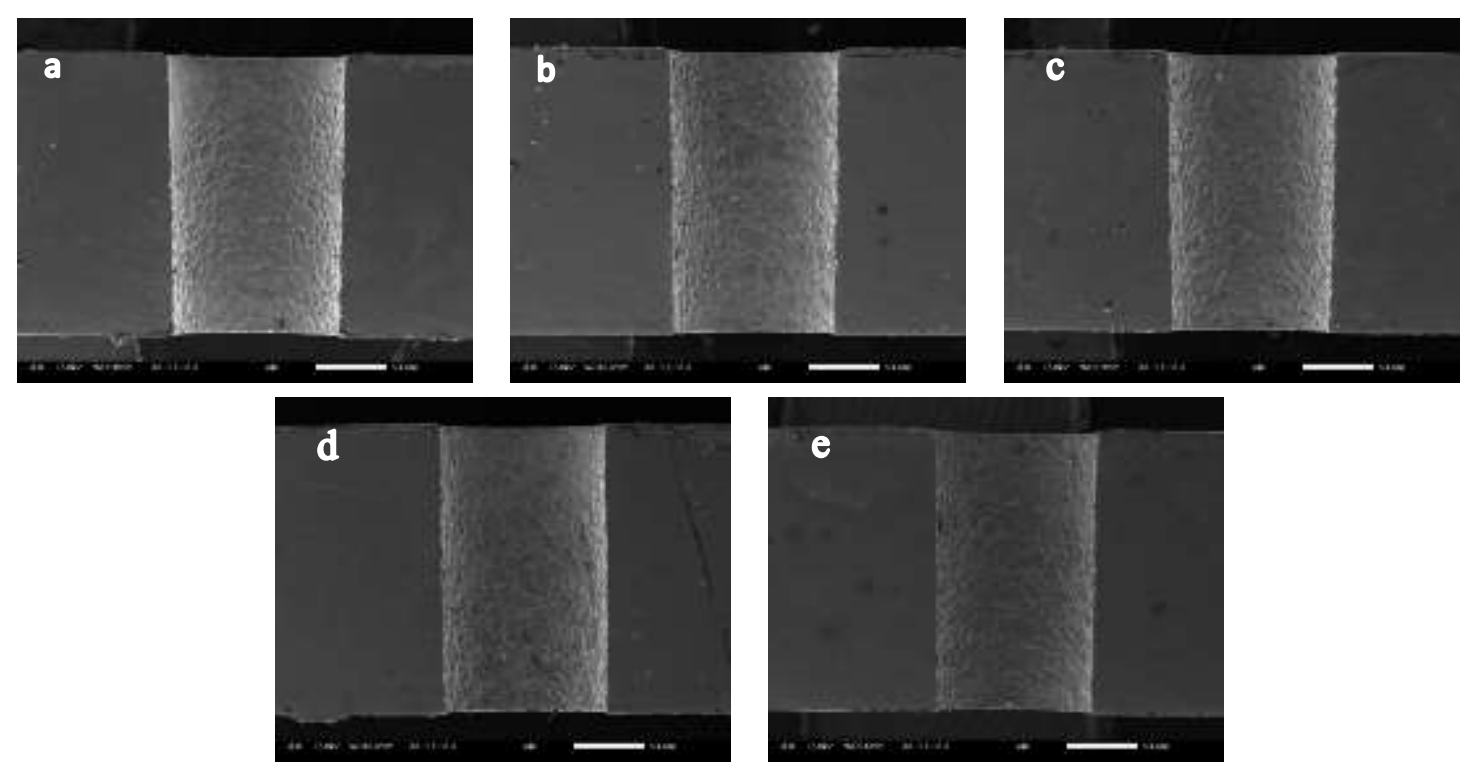

Fig. 20 The morphologies of the holes in the wall at different rotating speeds of the tool

electrode in high-speed EDM: (a) $60 \mathrm{rpm}$; (b) $160 \mathrm{rpm}$; (c) $260 \mathrm{rpm}$; (d) $360 \mathrm{rpm}$; (e) $460 \mathrm{rpm}$

\subsection{Influence of external flush pressure on ECM}

Fig. 21 shows the influence of external flushing pressure on machining performance in ECM. The curve shows that as the external flushing pressure increased, 
the MRR and exit diameter increased while the taper decreased. This indicates that when the direction of rotation of the helical electrode matched the external flushing in electrochemical machining, the electrolyte was extruded into helical groove owing to the high pressure. At the same time, a vortex was created around the groove that added axial speed to electrochemical products. The higher the pressure was at the entrance, the higher the volume of electrolyte flowing into the narrow gap, and the higher the accuracy of the exit and the MRR were, as shown in Fig. 22. It shows different shapes of the exit when using different external flushing pressures. However, a very high flush pressure could have influenced the stability of rotation, leading to a wide entrance, degrading surface quality, and increasing taper as shown in Fig. 23. It shows the morphology of the taper of the hole at different external flushing pressures. Hence, the most appropriate external flush pressure was $4 \mathrm{MPa}$, and yielded a hole with a diameter of $1.17 \mathrm{~mm}, \mathrm{MRR}$ of $0.039 \mathrm{~mm}^{3} / \mathrm{s}$, and taper of 0.01 . 

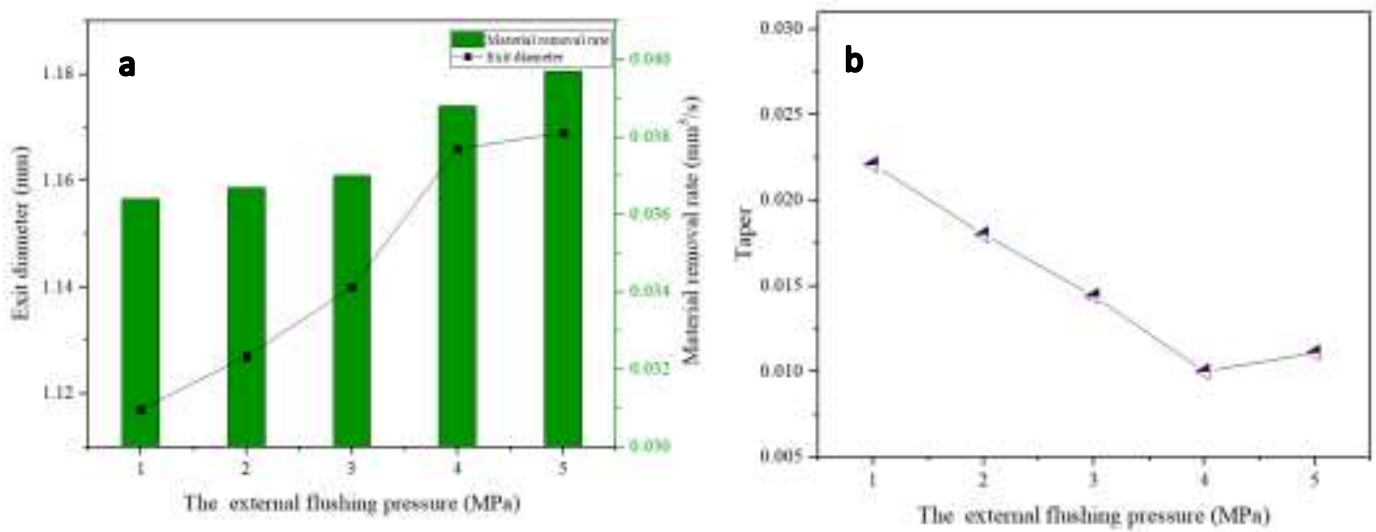

Fig. 21 Influence of external flushing pressure on machining performance in ECM:

(a ) diameter and MRR; (b) taper
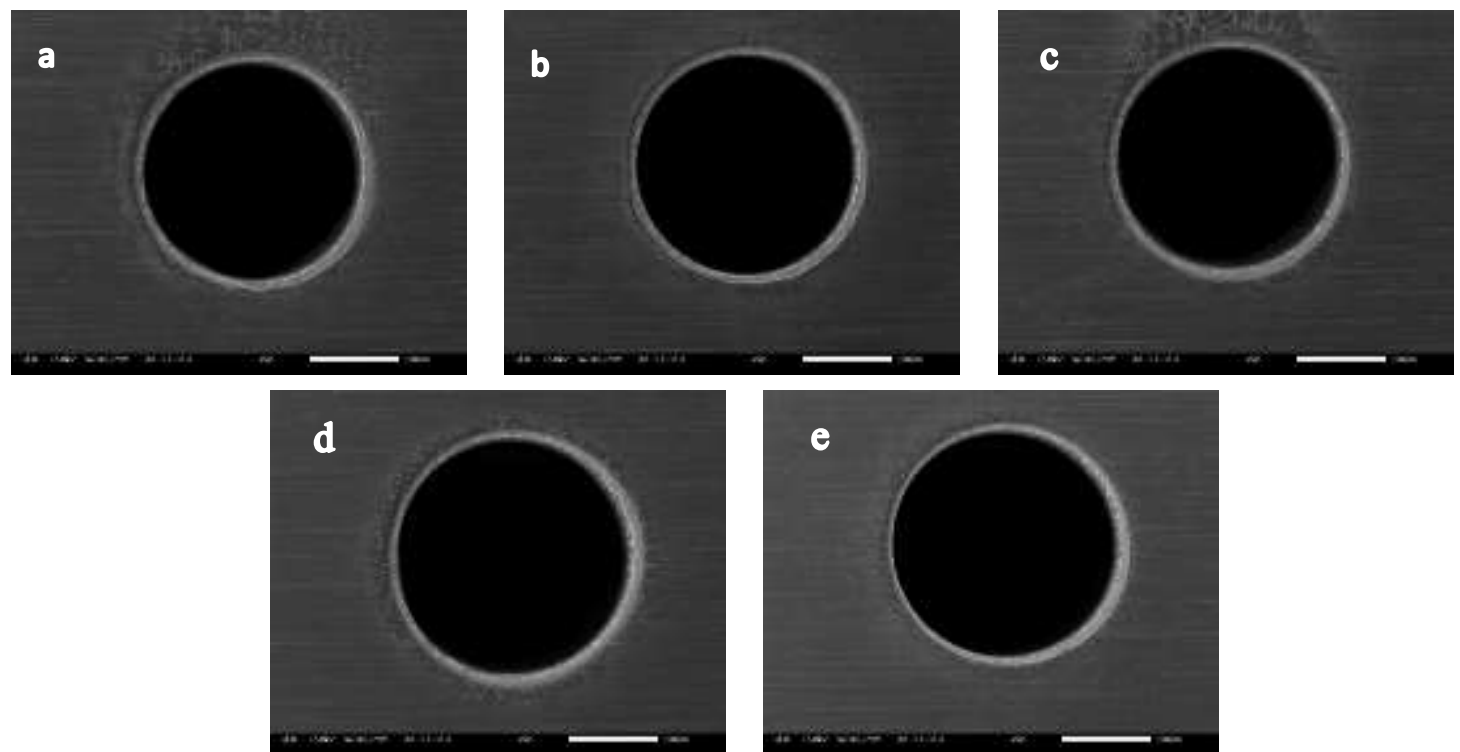

Fig. 22 The morphologies of the exit with different external flush pressures in ECM:

(a) $1 \mathrm{MPa}$; (b) $2 \mathrm{MPa}$; (c) $3 \mathrm{MPa}$; (d) $4 \mathrm{MPa}$; (e) $5 \mathrm{MPa}$ 

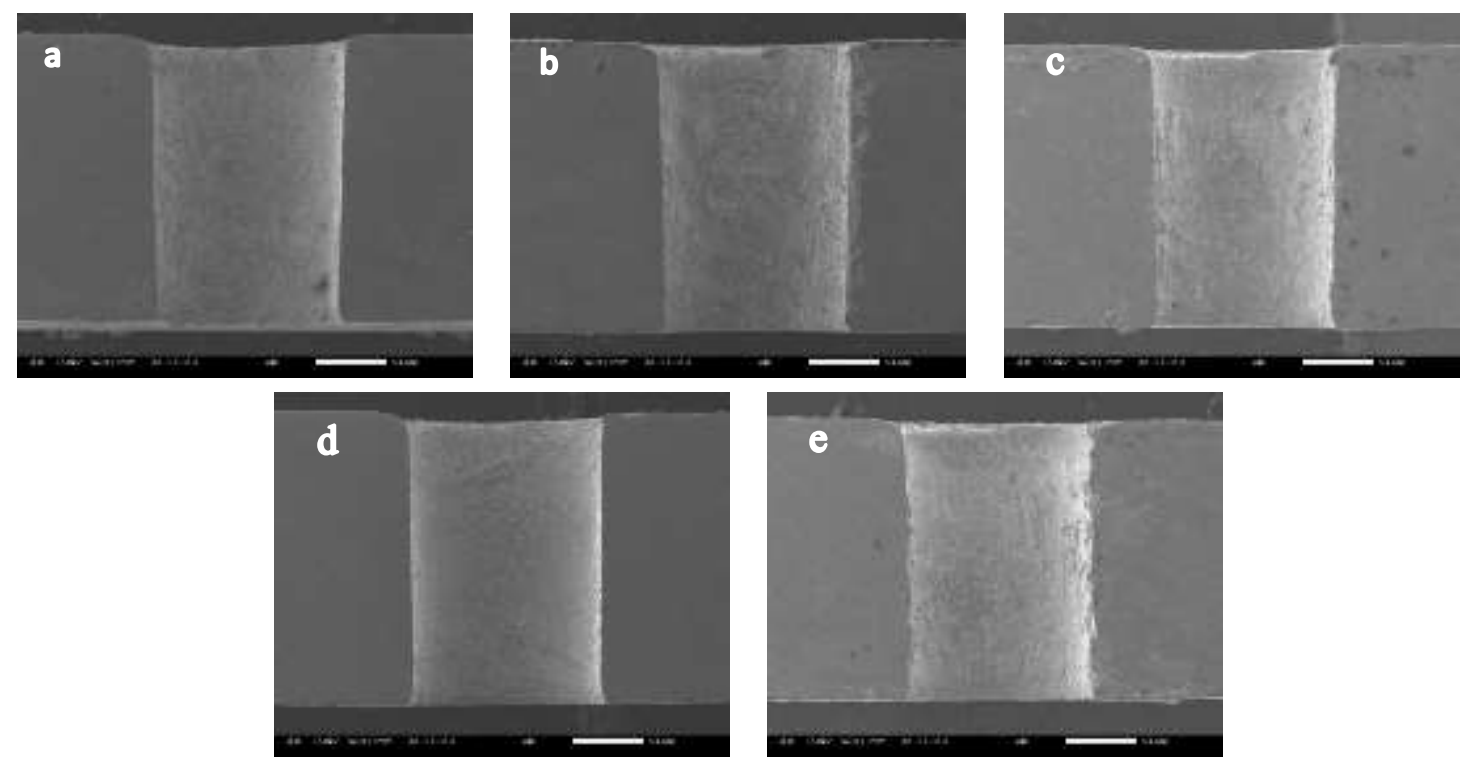

Fig. 23 The morphologies of the hole in the wall with different external flush pressures in ECM: (a) $1 \mathrm{MPa}$; (b) $2 \mathrm{MPa}$; (c) $3 \mathrm{MPa}$; (d) $4 \mathrm{MPa}$; (e) $5 \mathrm{MPa}$

\subsection{Influence of rotating speed of tool electrode on ECM}

Fig. 24 shows the influence of the rotating speed of helical electrode on the performance of ECM. The diameter of the exit and the MRR increased, and the maximum material removal rate was $0.014 \mathrm{~mm}^{3} / \mathrm{s}$. The taper decreased with increasing rotating speed. The higher speed added axial velocity to the machining products. The helical grooves expanded the flowing space of the electrolyte and refreshed it more quickly. This helped remove more material, which increased the MRR and improve the effect of electrolysis around the exit. This can be observed in the SEM images in Fig. 25. The electrochemical performance at entrance was better than that at the exit; thus, the taper decreased as the electrolytic reaction continued, as shown in Fig. 26. Increasing the velocity of the dielectric in the narrow space effected by high rotating speed refreshed the electrolyte and reduced the taper until a micro-hole with a small taper was obtained. The optimum rotating speed of the tool electrode was $460 \mathrm{rpm}$. 

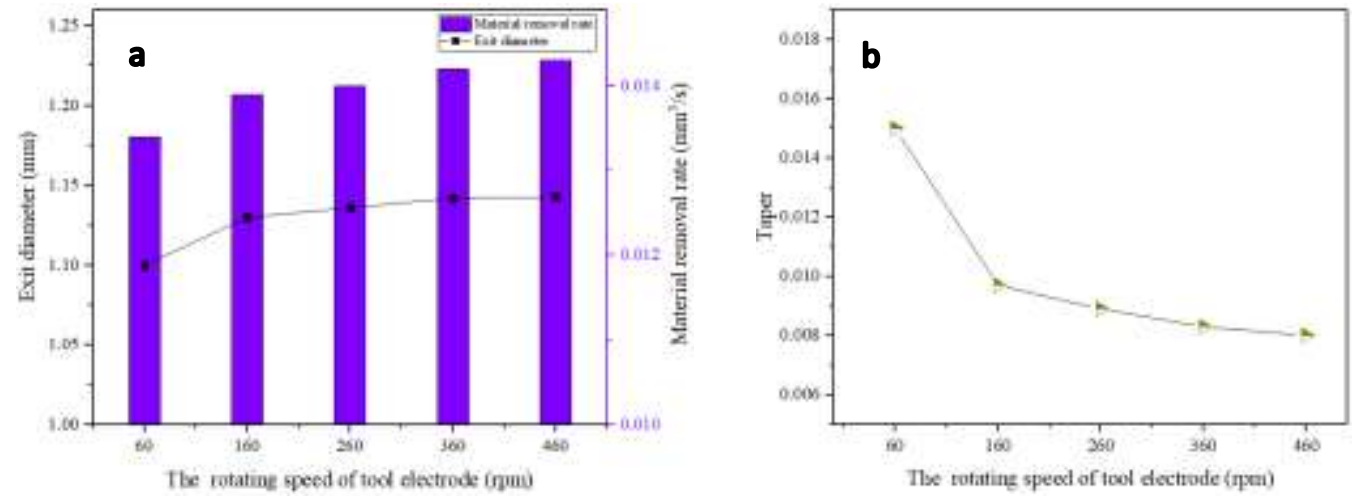

Fig. 24 Influence of the rotating speed of the tool electrode on machining performance in ECM: (a) diameter and MRR; (b) taper
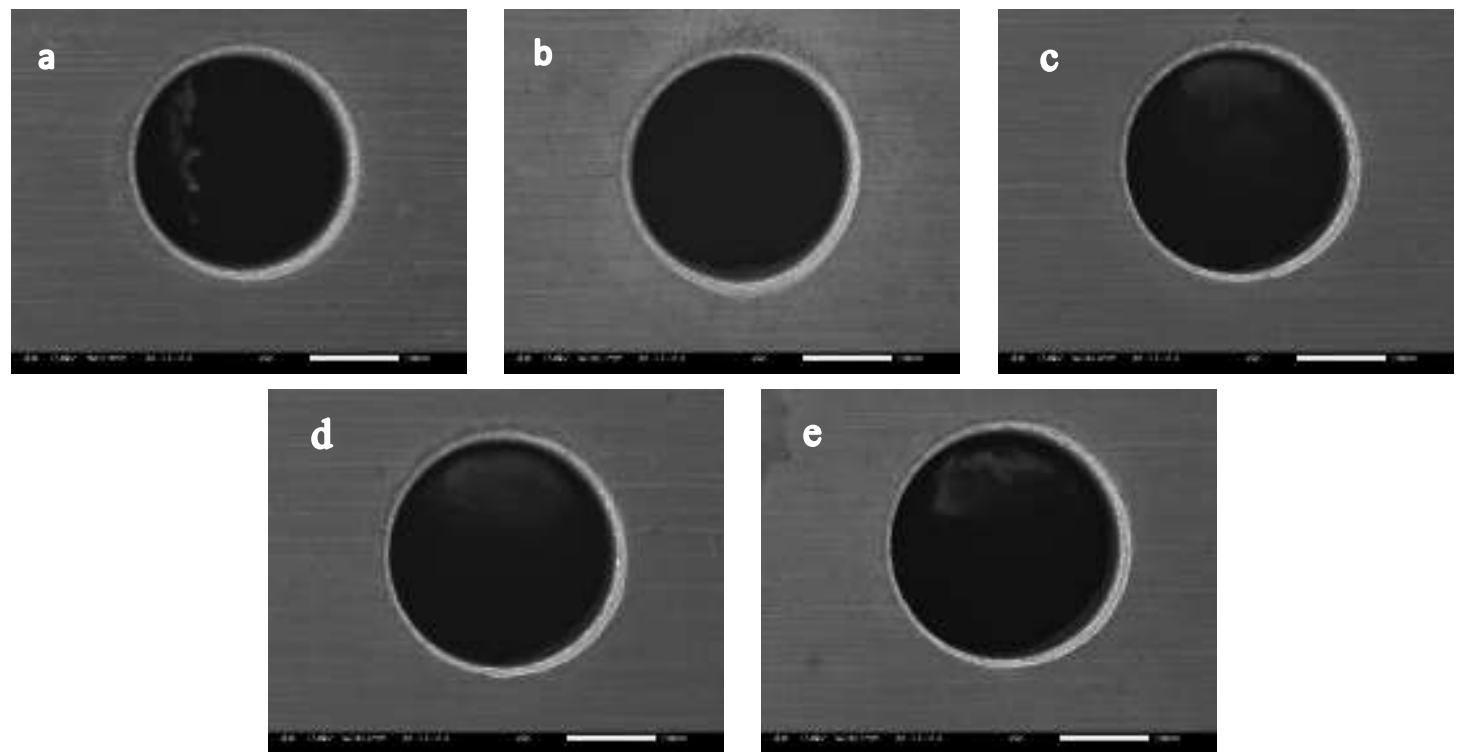

Fig. 25 The morphologies of the exit at different the rotating speeds of the tool electrode in ECM: (a) 60 rpm; (b) $160 \mathrm{rpm}$; (c) $260 \mathrm{rpm}$; (d) $360 \mathrm{rpm}$; (e) $460 \mathrm{rpm}$ 

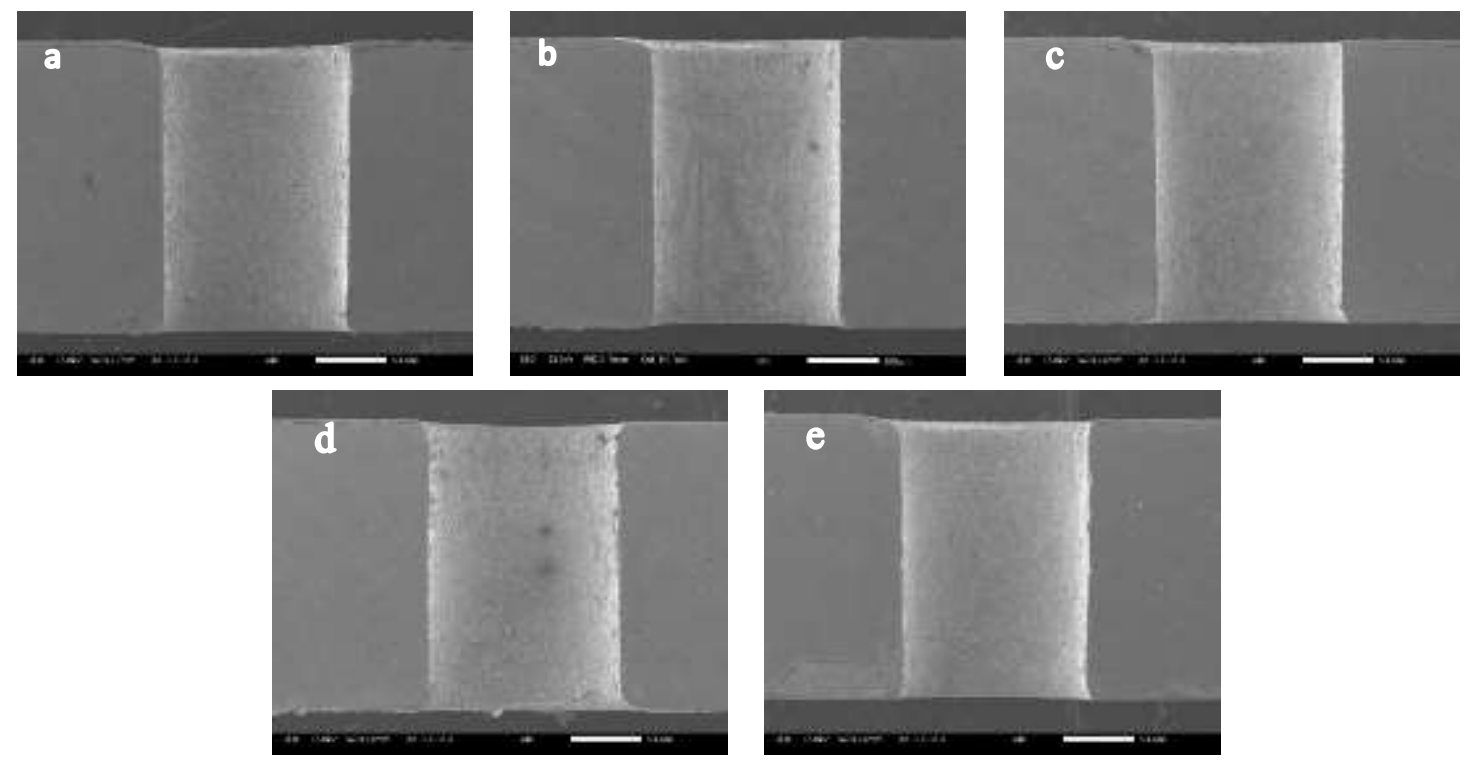

Fig. 26 The morphologies of the holes in the wall at different the rotating speeds of the tool electrode in ECM: (a) $60 \mathrm{rpm}$; (b) $160 \mathrm{rpm}$; (c) $260 \mathrm{rpm}$; (d) $360 \mathrm{rpm}$; (e) $460 \mathrm{rpm}$

\section{Conclusions}

This study proposed a combination of high-speed electrical discharge drilling and ECM by using a helical tube electrode matched with internal and external flushing to remove debris and enhance the flow velocity of the electrolyte in narrow gaps. This can help improve the accuracy of machining of micro-holes. The conclusions of this study can be summarized as follows:

- The results of simulations of the flow field showed that using a helical tube electrode, the directions of rotation of which matched the internal and external flushing, can enhance the flow velocity and reduce particle concentration in the narrow gap.

- Experiments to verify the results of the simulations showed that under the combination of high-speed EDM and ECM, drilling by using a helical tube electrode can yield a high machining accuracy, high surface quality, and a small taper hole.

- We used the proposed design in an experiment to optimize the speed of rotation of 
the tool electrode, pressures of the internal and external flushing, and the distance between the workpiece and the guide device. A minimum taper of 0.008 was thus obtained at a rotating speed of $460 \mathrm{rpm}$, and pressures of internal and external flushing of $9 \mathrm{MPa}$ and $4 \mathrm{MPa}$, respectively.

\section{Ethical Approval}

Not applicable.

\section{Consent to Participate}

Not applicable.

\section{Consent for publication}

Not applicable.

\section{Author Contributions}

Data curation, GQ. W.; Formal analysis, WT. Y.; Investigation, J.Z.; Methodology, Y.Z.; Project administration, Y.Z.; Writing original draft, L.J.; Writing review \& editing, Y.Z.

\section{Funding}

This research was funded by National Natural Science Foundation of China, grant number 51705239.

\section{Acknowledgement}

This project is supported by the National Natural Science Foundation of China (Grant No. 51705239)

\section{Conflicts of Interest}

The authors declare no competing interests.

\section{Availability of data and materials}

Not applicable. 


\section{References}

1. Zhang Y, Wen Z, Pei H, Yue Z (2019) Equivalent model of close-packed film cooling holes in nickel-based single crystal cooled blade based on crystallographic theory. Chin J Aeronaut 32: 839850. https://doi.org/10.1016/j.cja.2019.01.007

2. Wang J, Liang J, Wen Z, Yang Y, Yue Z (2019) The inter-hole interference on creep deformation behavior of nickel-based single crystal specimen with film-cooling holes. Int J Mech Sci 163:105090. https://doi.org/10.1016/j.ijmecsci.2019.105090

3. Wen, Z, Liang, J, Liu, C, Pei, H, Wen, S, Yue, Z (2018) Prediction method for creep life of thinwall specimen with film cooling holes in Ni-based single-crystal superalloy. Int J Mech Sci 141: 276-289. https://doi.org/10.1016/j.ijmecsci.2018.04.018

4. Zhu XD, Zhang JZ, Tan XM (2019) Numerical assessment of round-to-slot film cooling performances on a turbine blade under engine representative conditions. In. Commun Heat Mass Transfer 100: 98-110. https://doi.org/10.1016/j.icheatmasstransfer.2018.12.008

5. Skamniotis C, Cocks ACF (2021) Minimising stresses in double wall transpiration cooled components for high temperature applications. Int J Mech Sci 189:105983.

https://doi.org/10.1016/j.ijmecsci.2020.105983

6. Li W, Lu X, Li X, Ren J, Jiang H (2019) Wall thickness and injection direction effects on flat plate full-coverage film cooling arrays: Adiabatic film effectiveness and heat transfer coefficient. Int J Therm Sci 136: 172-181. https://doi.org/10.1016/j.ijthermalsci.2018.10.021

7. Liu K, Yang SF, Han JC (2014) Influence of Coolant Density on Turbine Blade Film-Cooling With Axial and Compound Shaped Holes. J Heat Transfer 136:44501.https://doi.org/10.1115/1.4025901 
8. Krewinkel R (2013) A review of gas turbine effusion cooling studies. Int J Heat Mass Transfer 66: 706-722. http://dx.doi.org/10.1016/j.ijheatmasstransfer.2013.07.071

9. Kim KM, Park JS, Lee DH, Lee TW, Cho HH (2011) Analysis of conjugated heat transfer, stress and failure in a gas turbine blade with circular cooling passages. Eng Fail Anal 18:1212-1222. https://doi.org/10.1016/j.engfailanal.2011.03.002

10. Zhang Y, Xu Z, Zhu D, Xing J (2015) Tube electrode high-speed electrochemical discharge drilling using low-conductivity salt solution. Int $\mathrm{J}$ Mach Tools Manuf 92:10-18. http://dx.doi.org/10.1016/j.ijmachtools.2015.02.011

11. Ma Y, Cheng G (2017) Forming property and broaching error prediction of a forged nickel-based superalloy turbine disc. Aerosp Sci Technol 62:55-64. http://dx.doi.org/10.1016/j.ast.2016.09.022 12. Liu NM, Chiang KT, Horng JT, Chen CC (2010) Modeling and analysis of the edge disintegration in the EDM drilling cobalt-bonded tungsten carbide. Int J Adv Manuf Technol 51: 587-598. https://doi.org/10.1007/s00170-010-2629-Z

13. Li Z, Tang J, Bai J (2020) A novel micro-EDM method to improve microhole machining performances using ultrasonic circular vibration (UCV) electrode. Int $\mathrm{J}$ Mech Sci 175:105574.https://doi.org/10.1016/j.ijmecsci.2020.105574

14. Li, G., Natsu, W., 2020. Realization of micro EDM drilling with high machining speed and accuracy by using mist deionized water jet. Precis. Eng. 61, 136-146. https://doi.org/10.1016/j.precisioneng.2019.09.016

15. Xu Y, Chen J, Jiang B, Liu Y, Ni J (2018) Experimental investigation of magnetohydrodynamic effect in electrochemical discharge machining. Int $J$ Mech Sci 142-143, 86-96. https://doi.org/10.1016/j.ijmecsci.2018.04.020 
16. Kurita T, Hattori M (2006) A study of EDM and ECM/ECM-lapping complex machining $\begin{array}{llllll}\text { technology. } & \text { Int } & \mathrm{J} & \text { Mach } & \text { Tools } & \text { Manuf }\end{array}$ 46:18041810.https://doi.org/10.1016/j.ijmachtools.2005.11.009

17. Nguyen KH, Lee PA, Kim BH (2015) Experimental investigation of ECDM for fabricating micro structures of quartz. Int J Precis Eng Manuf 16:5-12. https://doi.org/10.1007/s12541-015$\underline{0001-9}$

18. Wang Y, Xu Z, Liu J, Zhang A, Xu Z, Meng D, Zhao J (2021) Study on flow field of electrochemical machining for large size blade. Int $J$ Mech Sci 190:106018.https://doi.org/10.1016/j.ijmecsci.2020.106018

19. Liang W, Tong H, Li Y, Li B (2019) Tool electrode wear compensation in block divided EDM process for improving accuracy of diffuser shaped film cooling holes. Int J Adv Manuf Technol 103:1759-1767. https://doi.org/10.1007/s00170-019-03591-8

20. Liu K, Lauwers B, Reynaerts D (2009) Process capabilities of Micro-EDM and its applications. Int J Adv Manuf Technol 47:11-19.https://doi.org/10.1007/s00170-009-2056-1

21. Pham DT, Ivanov A, Bigot S, Popov K, Dimov S (2006) An investigation of tube and rod electrode wear in micro EDM drilling. Int $\mathrm{J}$ Adv Manuf Technol 33:103-109. https://doi.org/10.1007/s00170-006-0639-7

22. Zhang Y, Xu Z, Zhu Y, Zhu D (2016) Effect of tube-electrode inner structure on machining performance in tube-electrode high-speed electrochemical discharge drilling. J Mater Process Technol 231:38-49. http://dx.doi.org/10.1016/j.jmatprotec.2015.12.012

23. Fang XL, Zou XH, Chen M, Zhu D (2017) Study on wire electrochemical machining assisted with large-amplitude vibrations of ribbed wire electrodes. CIRP Ann-Manuf 66:205-208. 
http://dx.doi.org/10.1016/j.cirp.2017.04.135

24. Zou XH, Fang XL, Zeng YB, Zhu D (2017) A high efficiency approach for wire electrochemical micromachining using cutting edge tools. Int $\mathrm{J}$ Adv Manuf Technol 91:3943-3952. https://doi.org/10.1007/s00170-017-0063-1

25. Plaza S, Sanchez JA, Perez E, Gil R, Izquierdo B, Ortega N, Pombo I (2014) Experimental study on micro EDM-drilling of Ti6Al4V using helical electrode. Precis Eng 38:821-827. http://dx.doi.org/10.1016/j.precisioneng.2014.04.010

26. Yang CK, Wu KL, Hung JC, Lee SM, Lin JC, Yan BH (2011) Enhancement of ECDM efficiency and accuracy by spherical tool electrode. Int $\mathrm{J}$ Mach Tools Manuf 51:528-535. https://doi.org/10.1016/j.ijmachtools.2011.03.001

27. Wang, W., Zhu, D., Qu, N.S., Huang, S.F., Fang, X.L., 2010. Electrochemical drilling with vacuum extraction of electrolyte. J. Mater. Process. Technol. 210, 238-244. https://doi.org/10.1016/j.jmatprotec.2009.09.006

28. Zhu D, Wang W, Fang XL, Qu NS, Xu ZY (2010) Electrochemical drilling of multiple holes with electrolyte-extraction. CIRP Ann $\quad$ Manuf 59:239-242. https://doi.org/10.1016/j.cirp.2010.03.135

29. Li C, Zhang B, Li Y, Tong H, Ding S, Wang Z, Zhao L (2018) Self-adjusting EDM/ECM high speed drilling of film cooling holes. J Mater Process Tech 262:95-103. https://doi.org/10.1016/j.jmatprotec.2018.06.026

30. Zhang Y, Xu Z, Zhu D, Xing J (2015) Tube electrode high-speed electrochemical discharge drilling using low-conductivity salt solution. Int $J$ Mach Tools Manuf 92:10-18. http://dx.doi.org/10.1016/j.ijmachtools.2015.02.011 


\section{Figures}
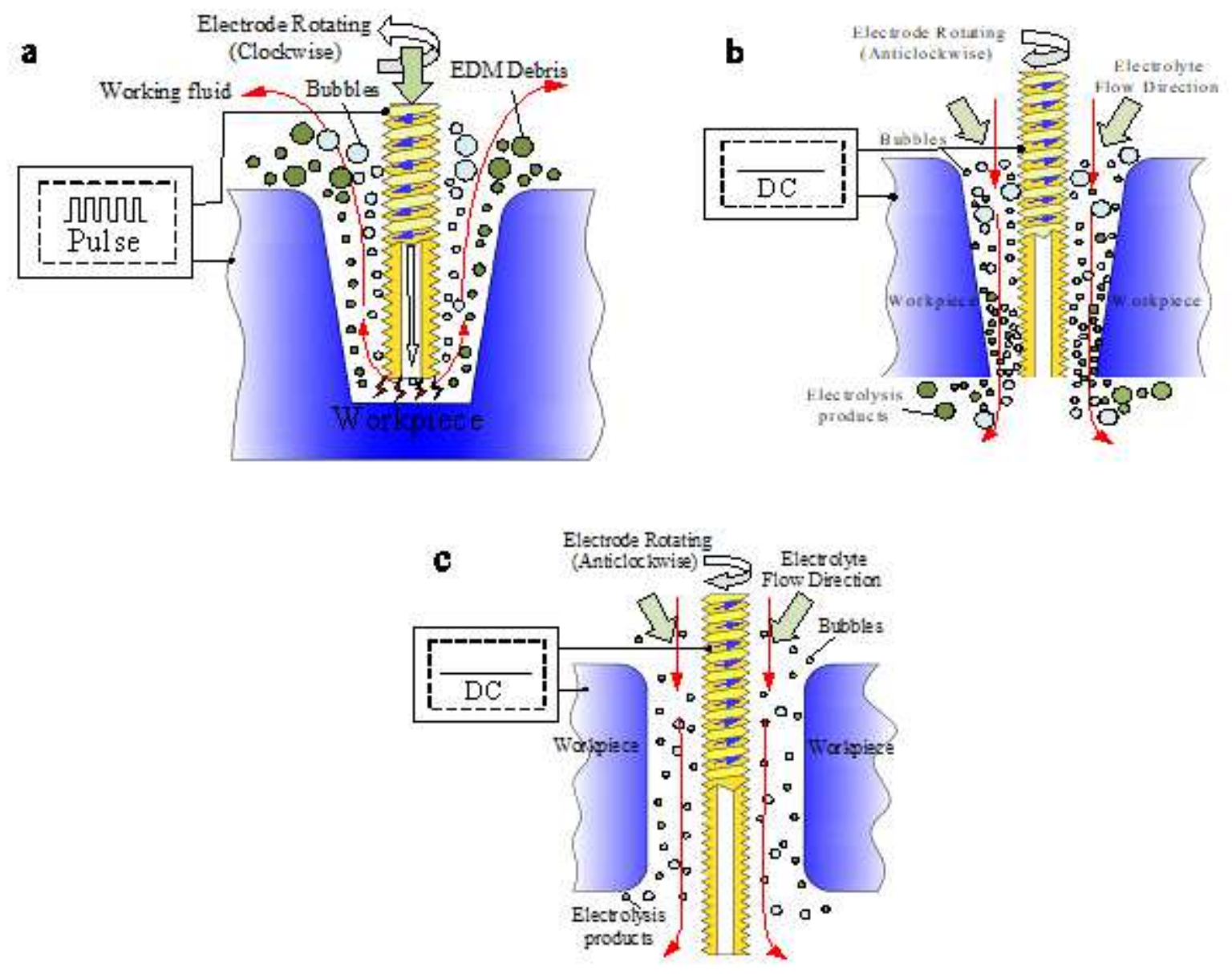

\section{Figure 1}

Principle of the combination of high-speed EDM and ECM: (a) high-speed electrical discharge drilling; (b) $\mathrm{ECM}$; (c) final results of machining 
a

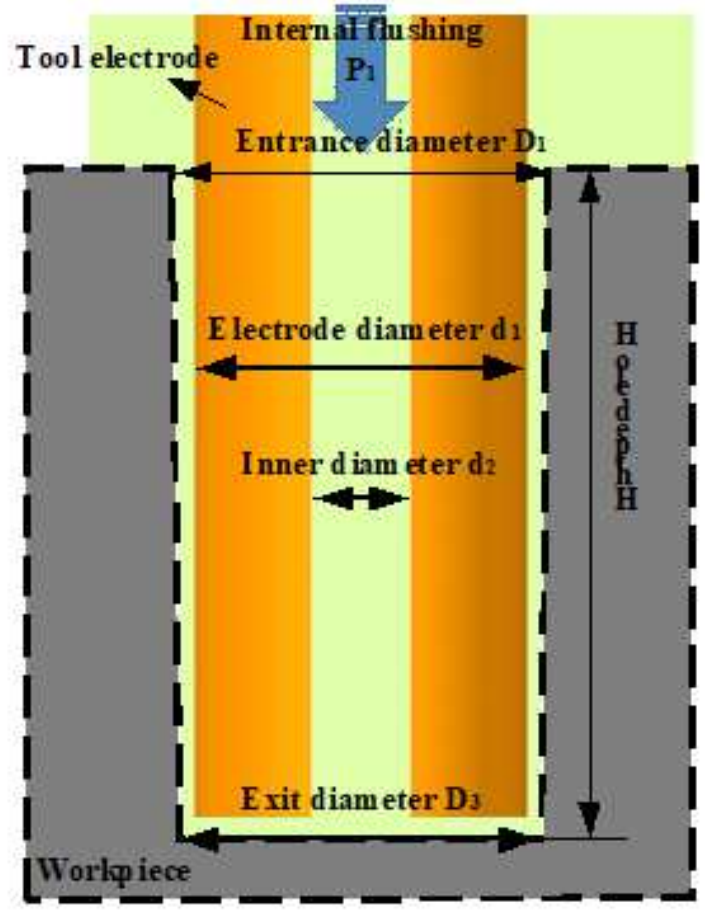

c

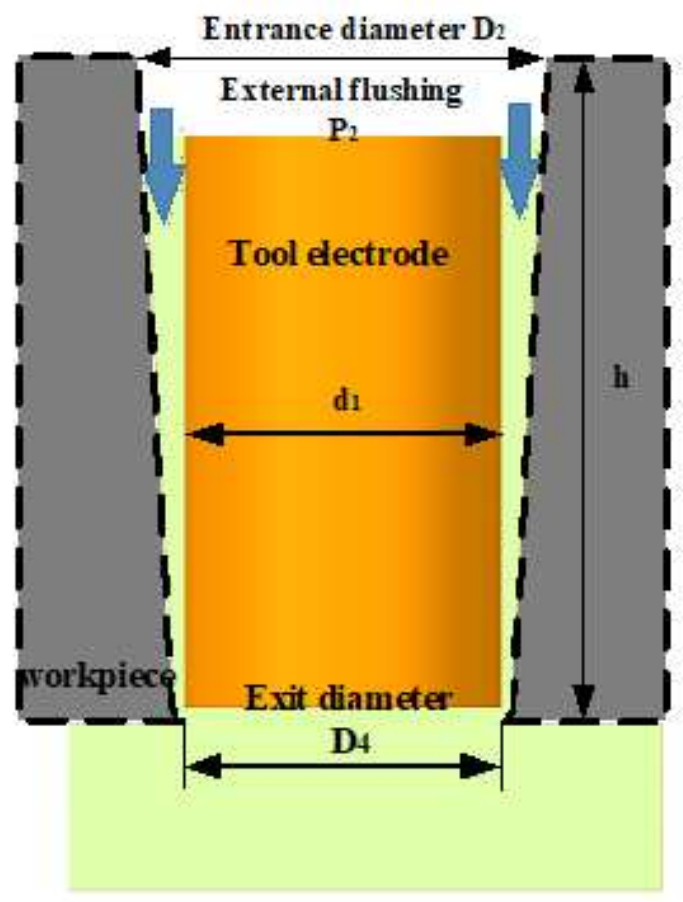

b

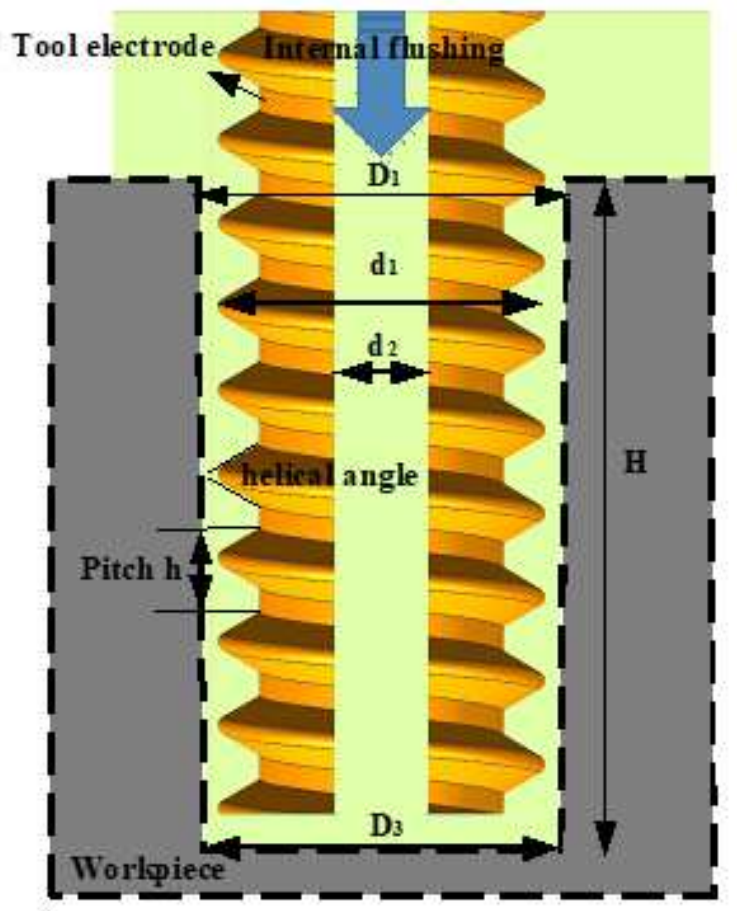

d

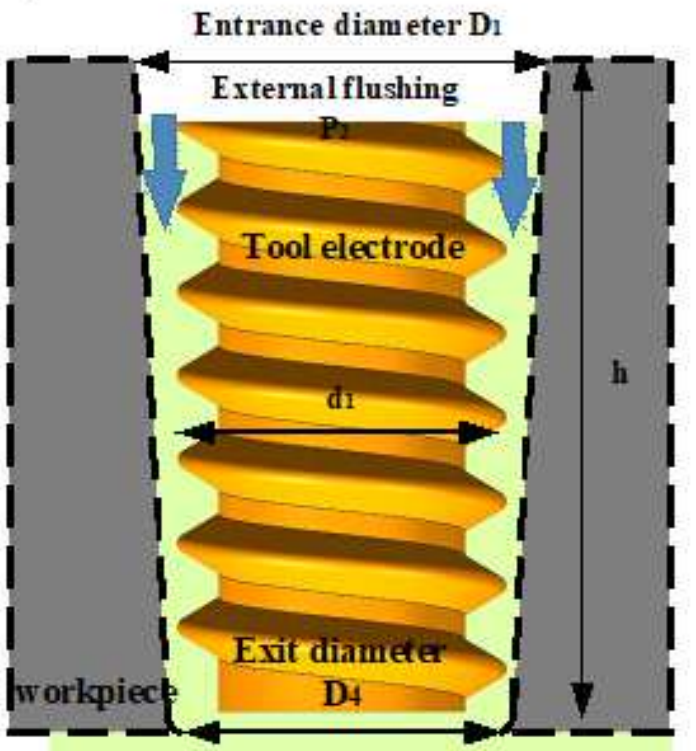

Figure 2

CFD models of the blind hole stage and breakthrough hole stage: (a) and (c) cylindrical tube electrode; (b) and (d) helical tube electrode 


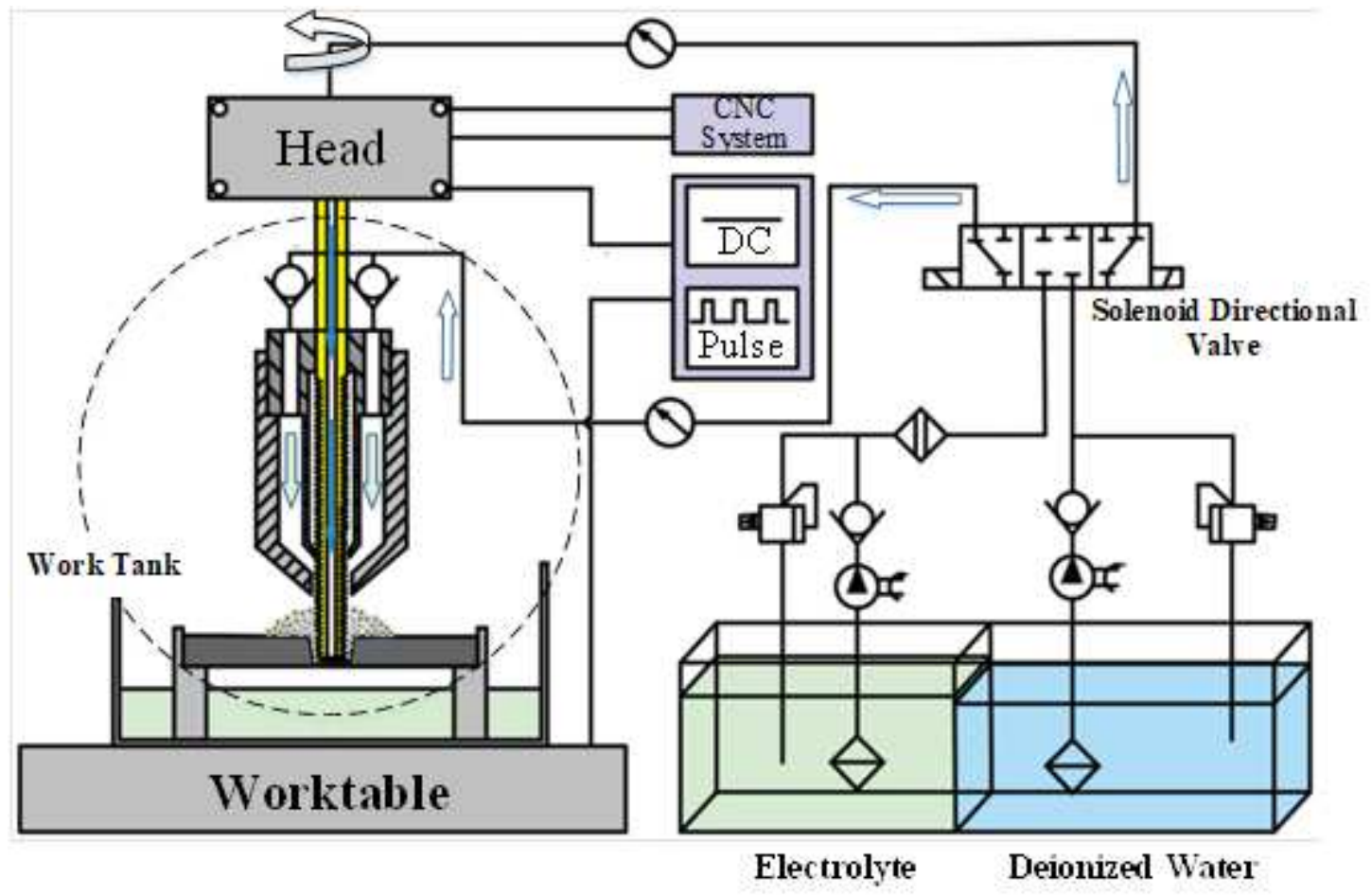

Figure 3

Experimental apparatus of a combination of high-speed EDM and ECM using a helical tube electrode with matched internal and external flushing
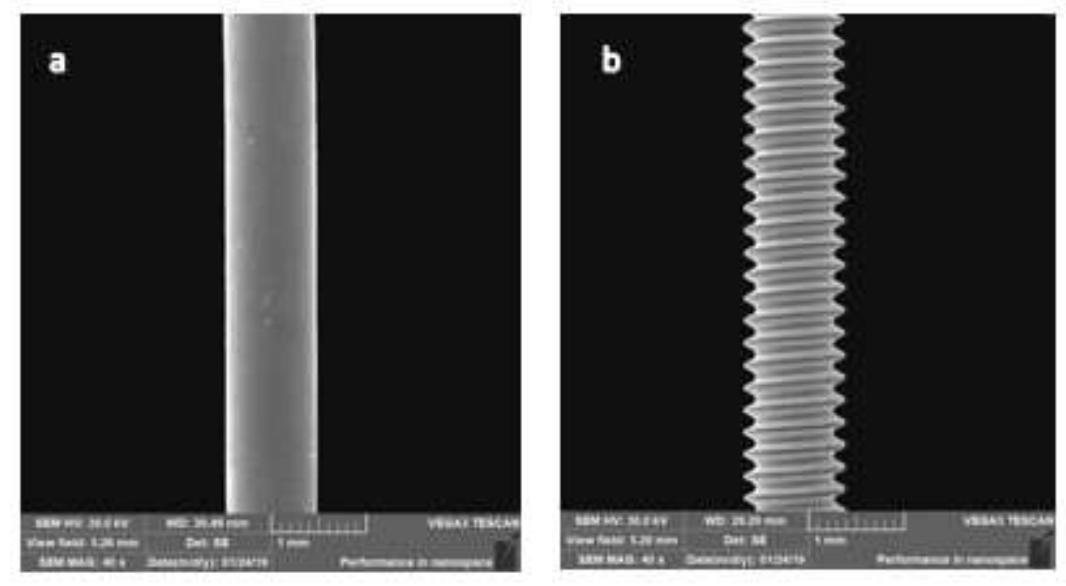

Figure 4

SEM images of the tube electrode fabricated with a smooth surface and a helical structure 
a
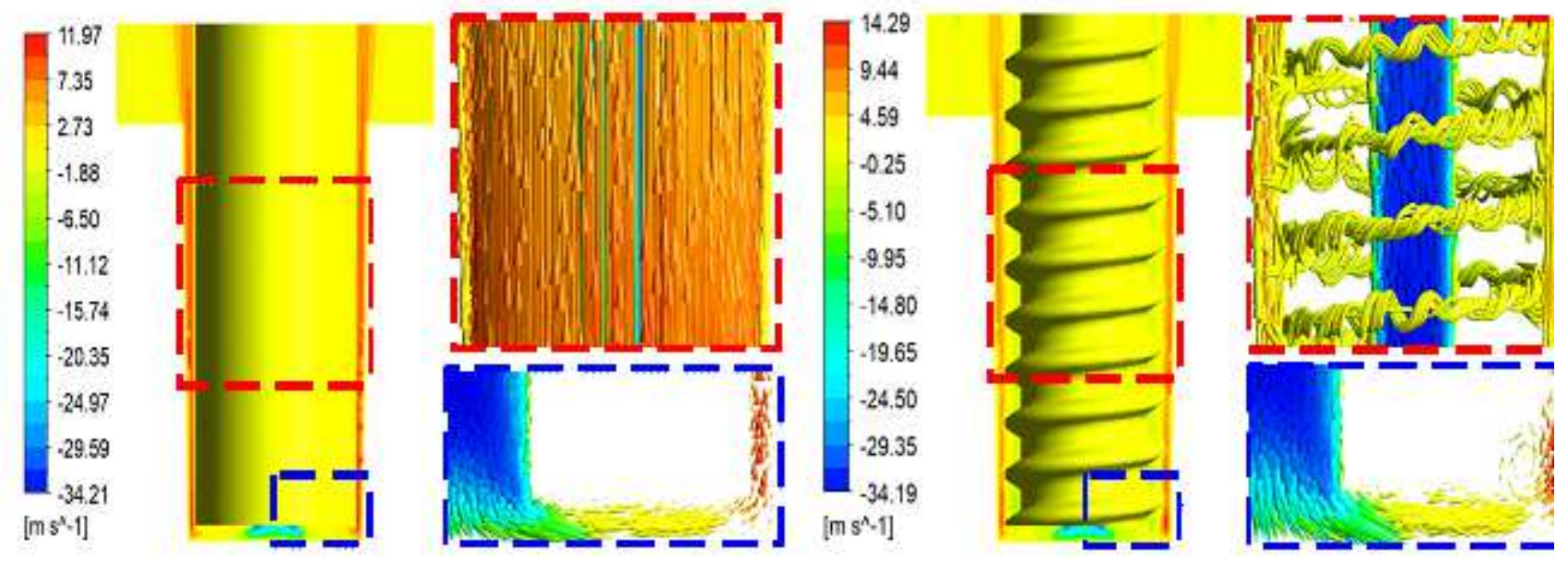

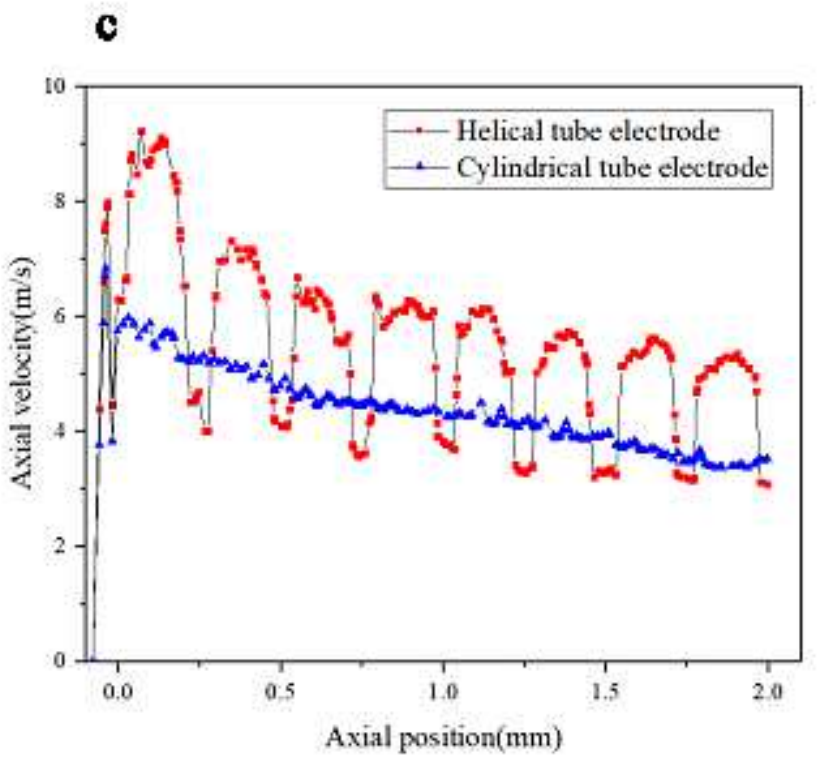

Figure 5

Contours of axial velocity: (a) cylindrical tube electrode; (b) helical tube electrode; (c) the velocity of the working fluid along the depth direction 
a

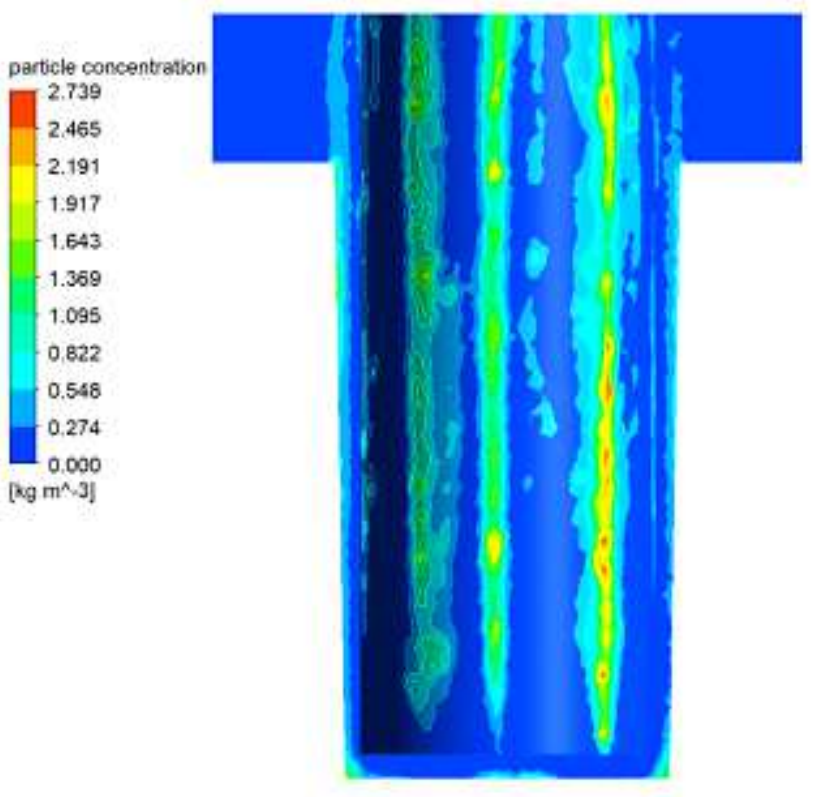

c

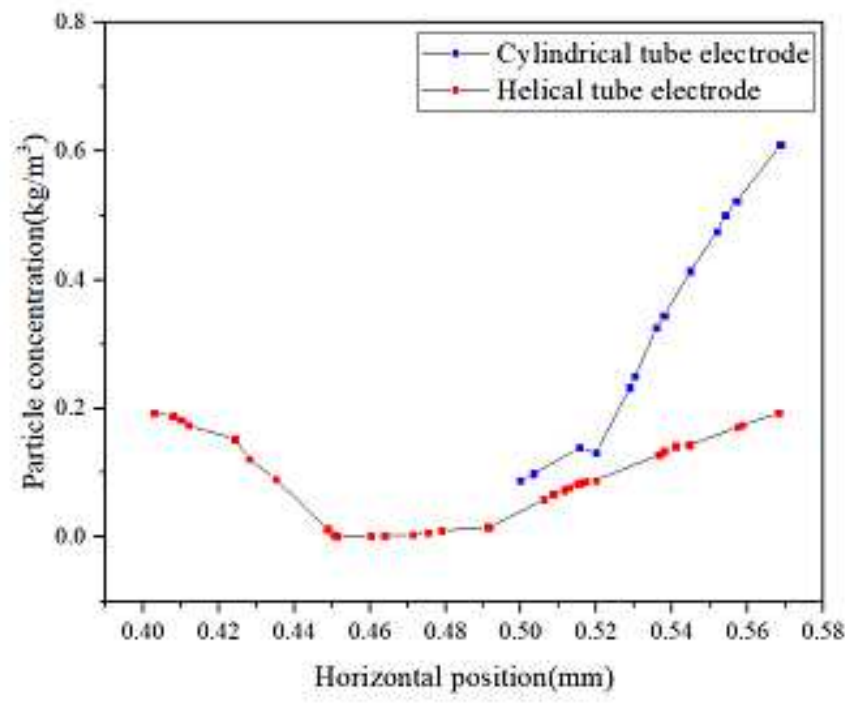

b

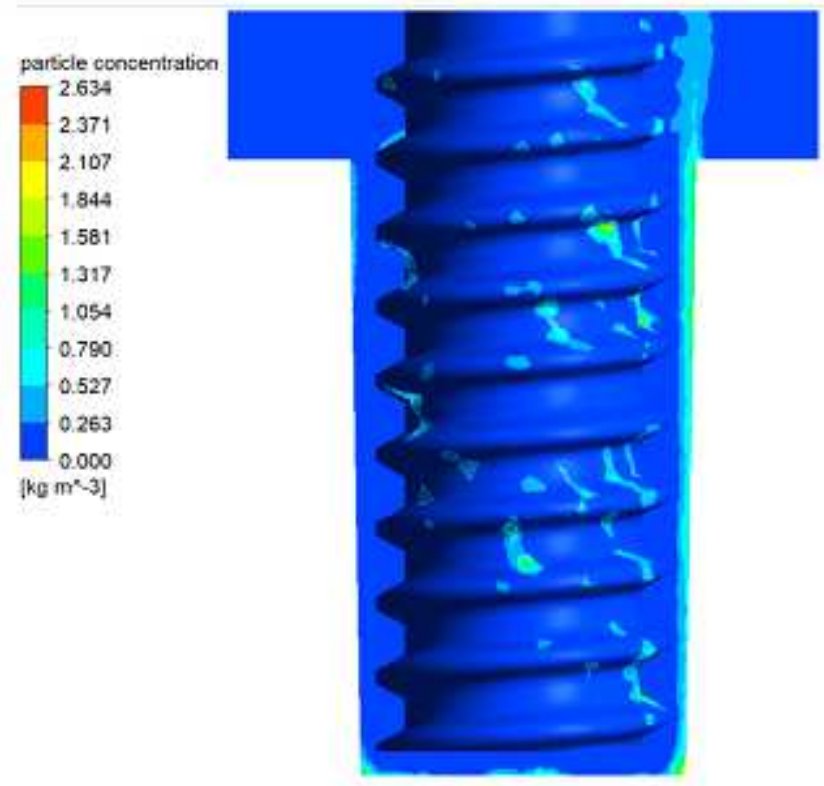

d

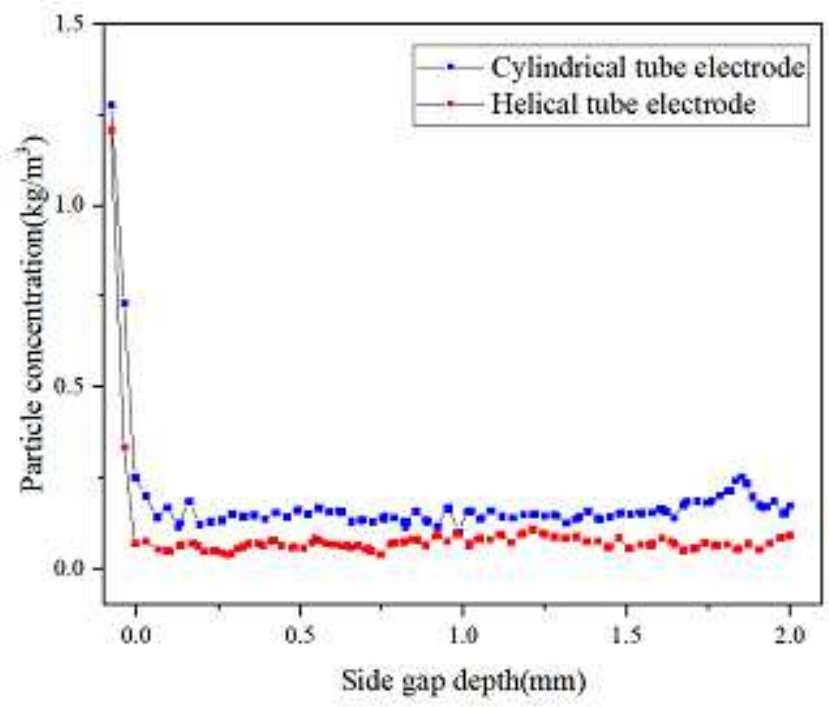

\section{Figure 6}

Cloud diagram of particle concentrations in the gap in case of (a) cylindrical tube electrode; and (b) helical tube electrode. Distribution of particle concentration in the helical groove and the side gap (c) and (d) 


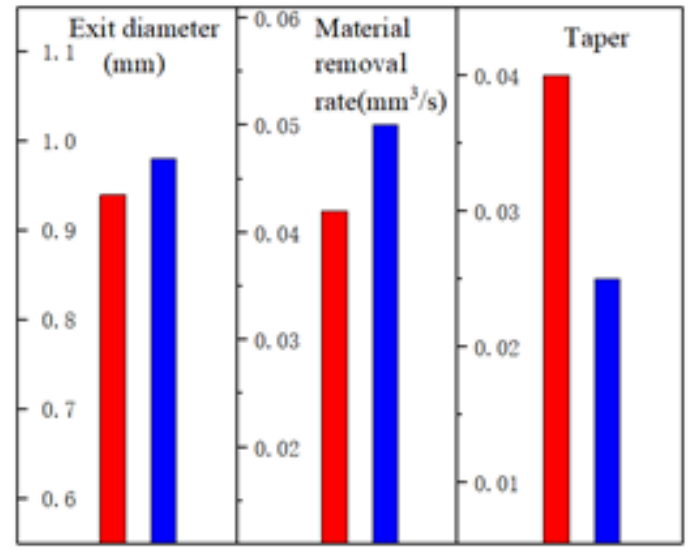

Cylindrical electrode

Helical electrode

\section{Figure 7}

Effects of electrode structure on the exit diameter, MRR, and taper of hole in case of high-speed EDM
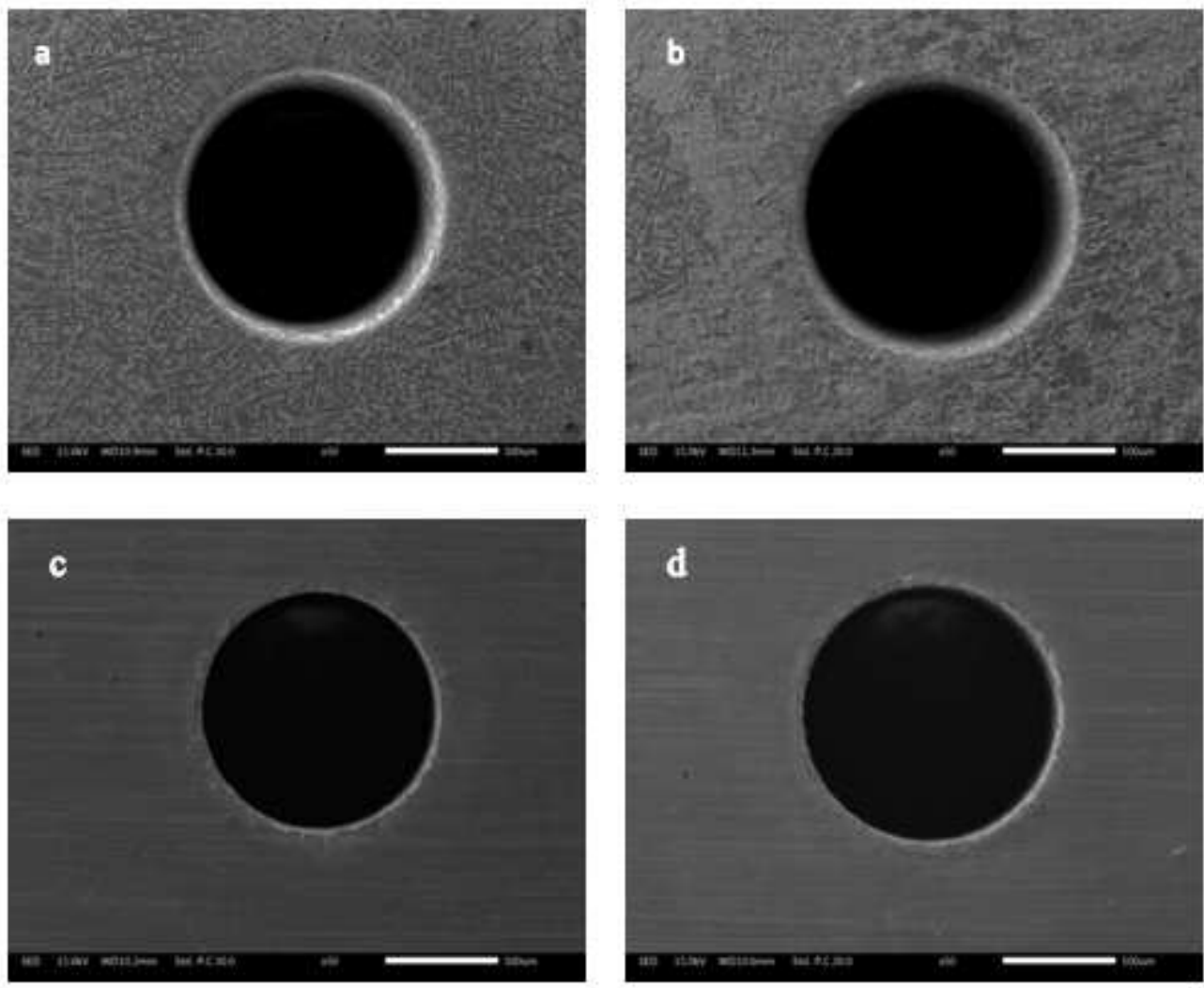

Figure 8

Morphologies of the entrance and the exit in high-speed EDM: (a) entrance, cylindrical tube; (c) exit, cylindrical tube electrode; (b) entrance, helical tube electrode; and (d) exit, helical tube electrode 

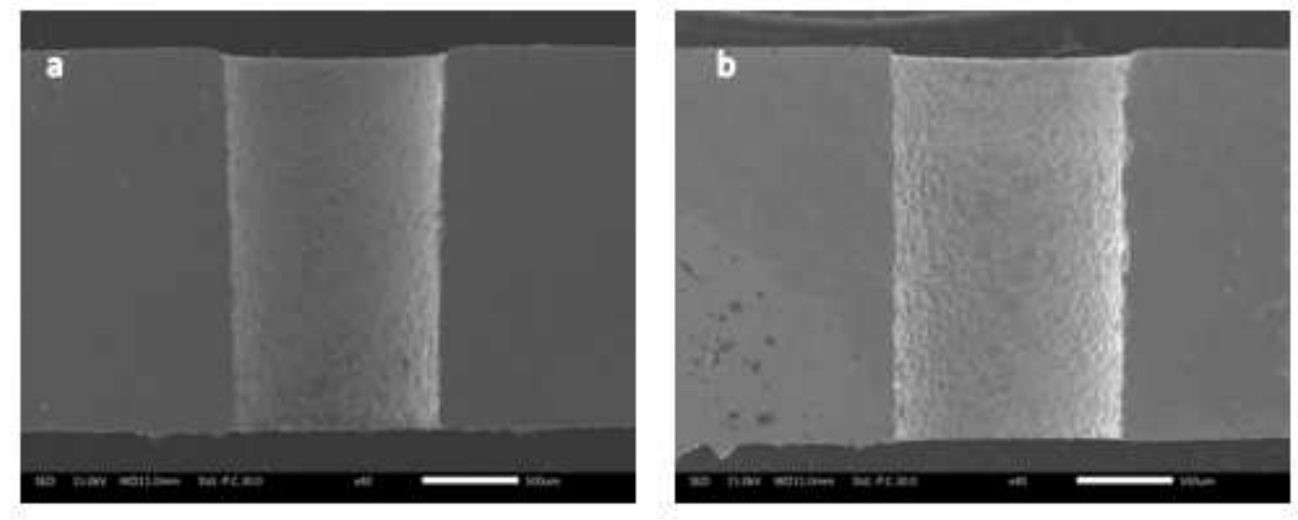

Figure 9

The morphology of the hole in the wall in high-speed EDM: (a) cylindrical tube electrode; (b) helical tube electrode 
a

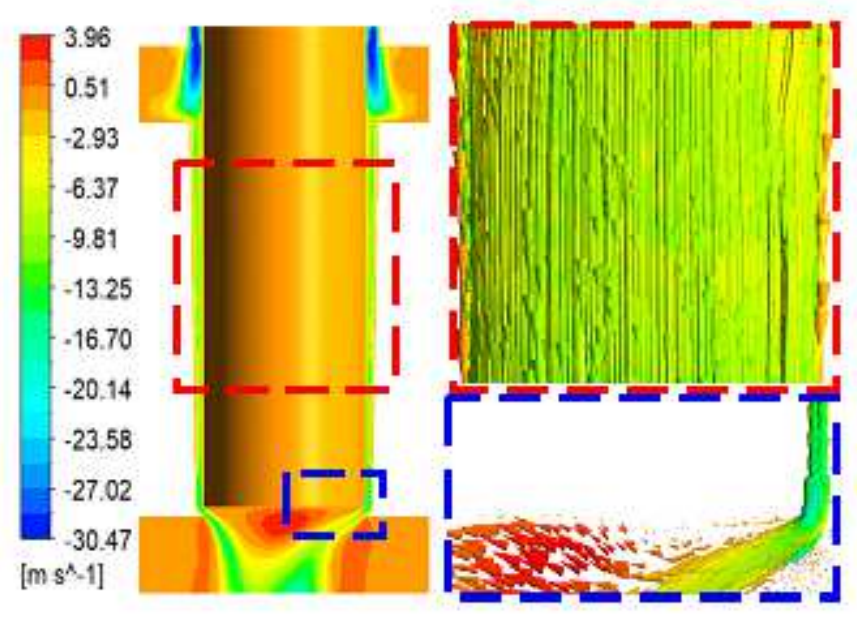

b

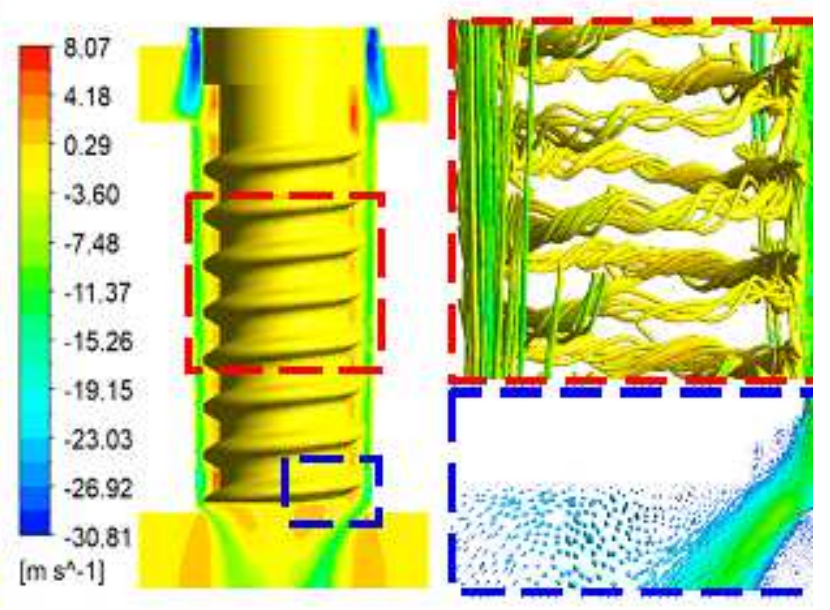

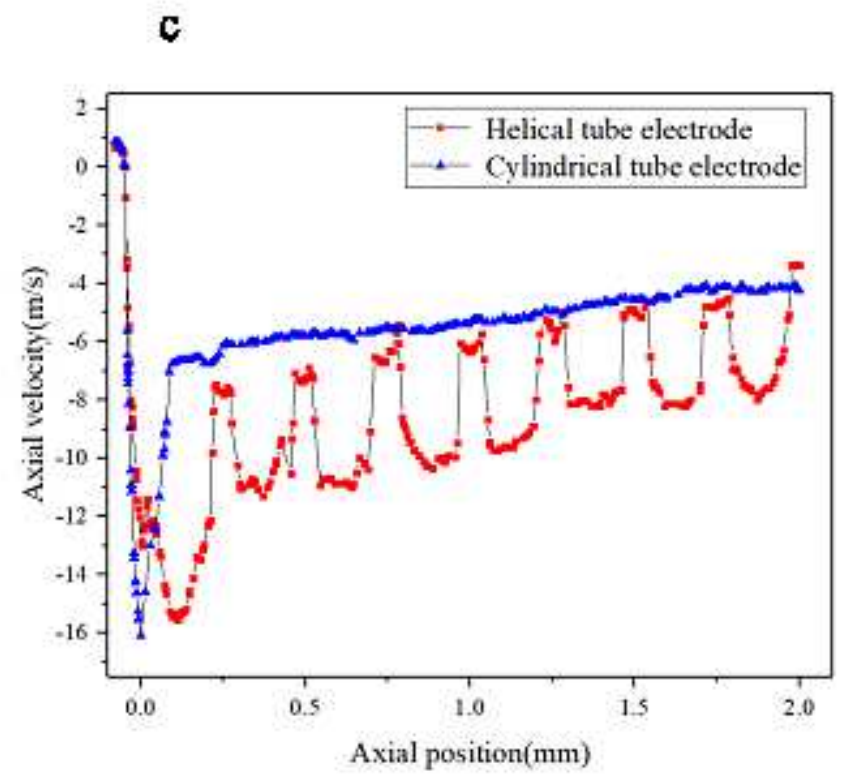

Figure 10

Contours of axial velocity in the breakthrough hole stage: (a) cylindrical tube electrode; (b) helical tube electrode; (c) velocity of working fluid in the direction of hole depth 
2

Particle Mass Concentration

\section{Contour 1}

11.89

10.70

$-9.51$

8.32

$-7.13$

5.94

$-4.75$

3.57

$-2.38$

$-1.19$

0.00

[kg mn]
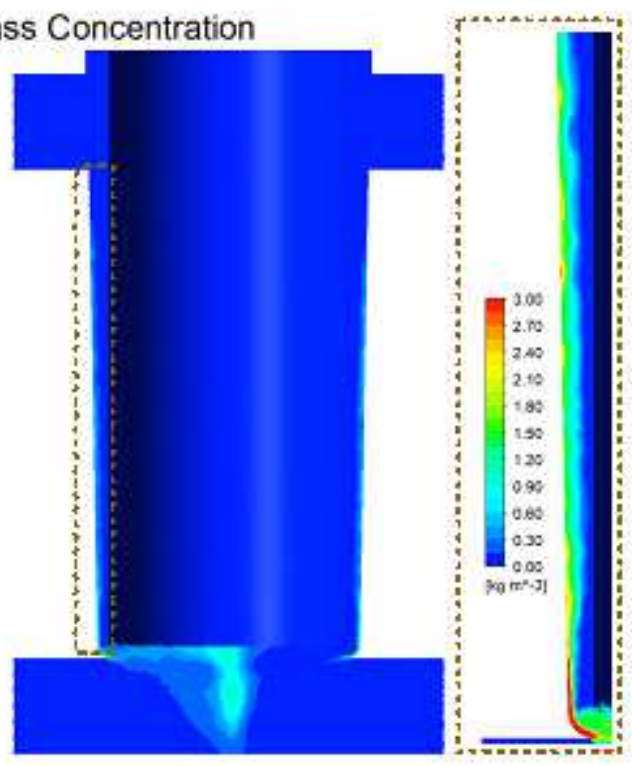

c

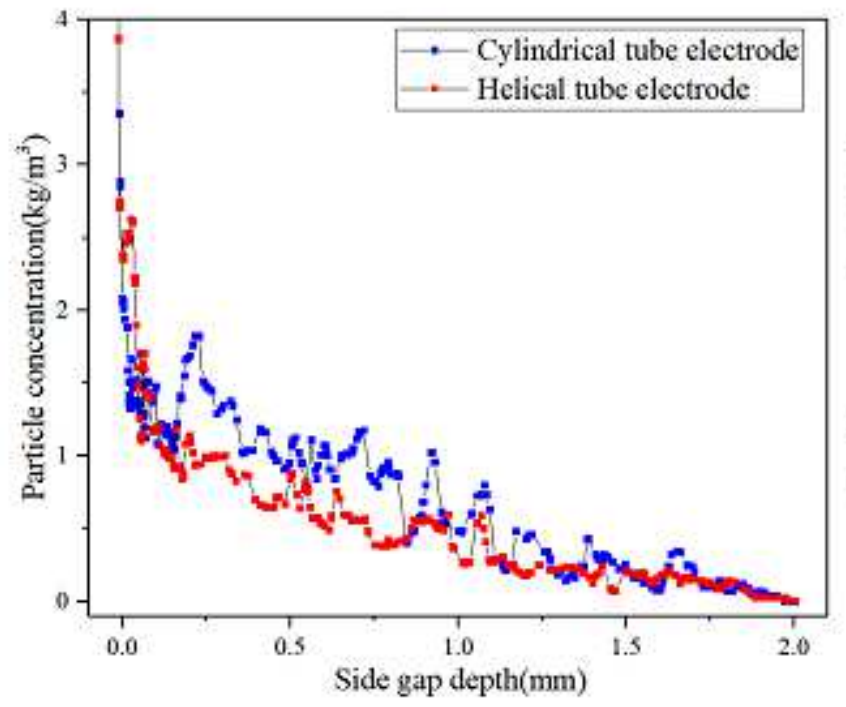

b

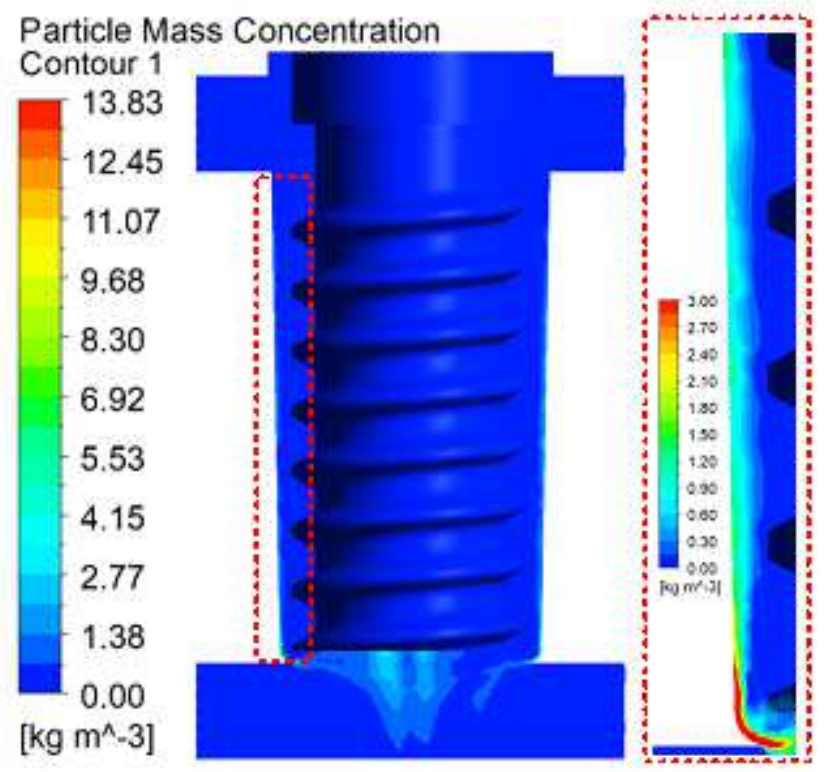

d

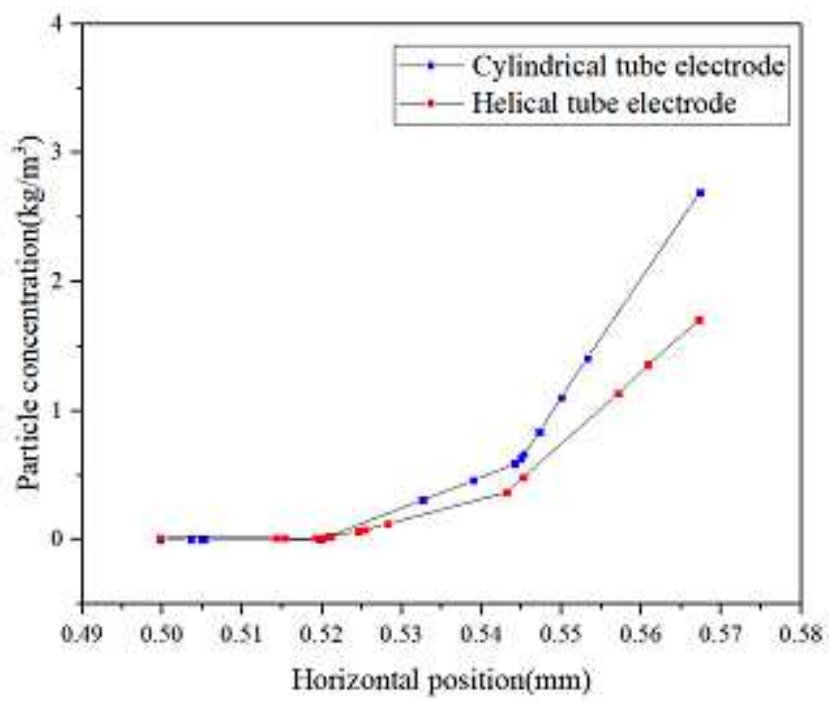

Figure 11

Cloud diagram of particle concentration in ECM, using: (a) cylindrical tube electrode; and (b) helical tube electrode. Particle concentration distribution in the helical groove and the side gap in ECM, (c) and (d) 


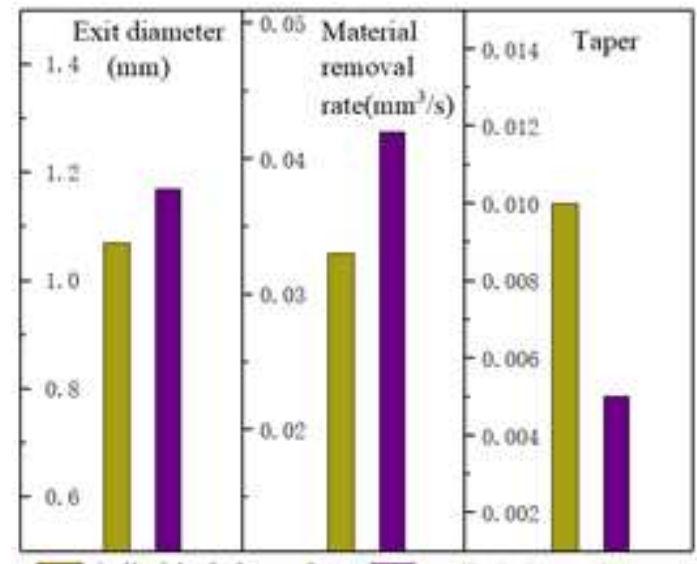

Cylindrical electrode

Helical electrode

\section{Figure 12}

Effects of the electrode structure on the diameter of the exit, the MRR, and the taper in ECM
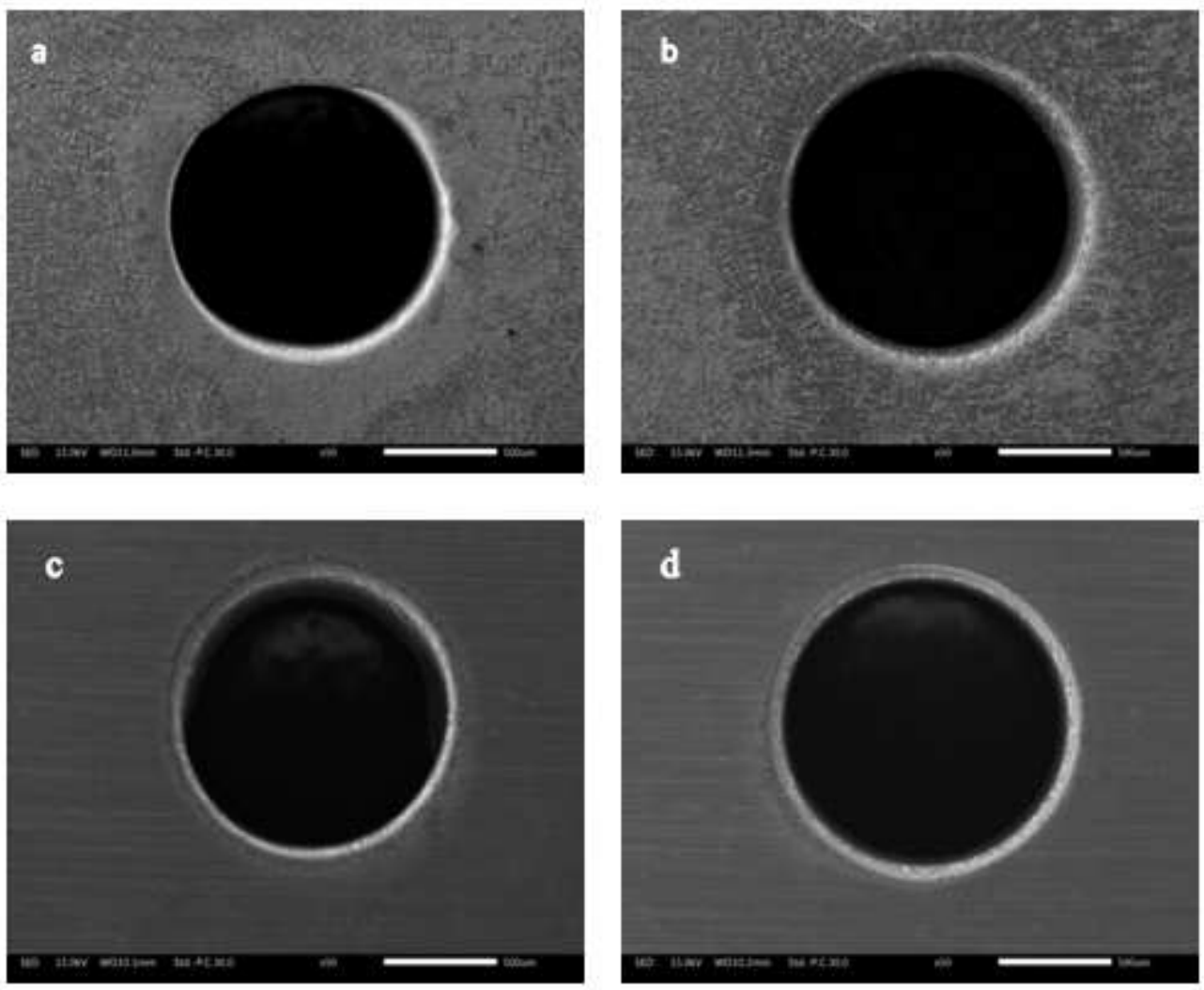

\section{Figure 13}

Influence of electrode structure on the roundness of the entrance and exit in ECM: (a) entrance, cylindrical electrode; (b) entrance, helical electrode; (c) exit, cylindrical electrode; (d) exit, helical electrode 


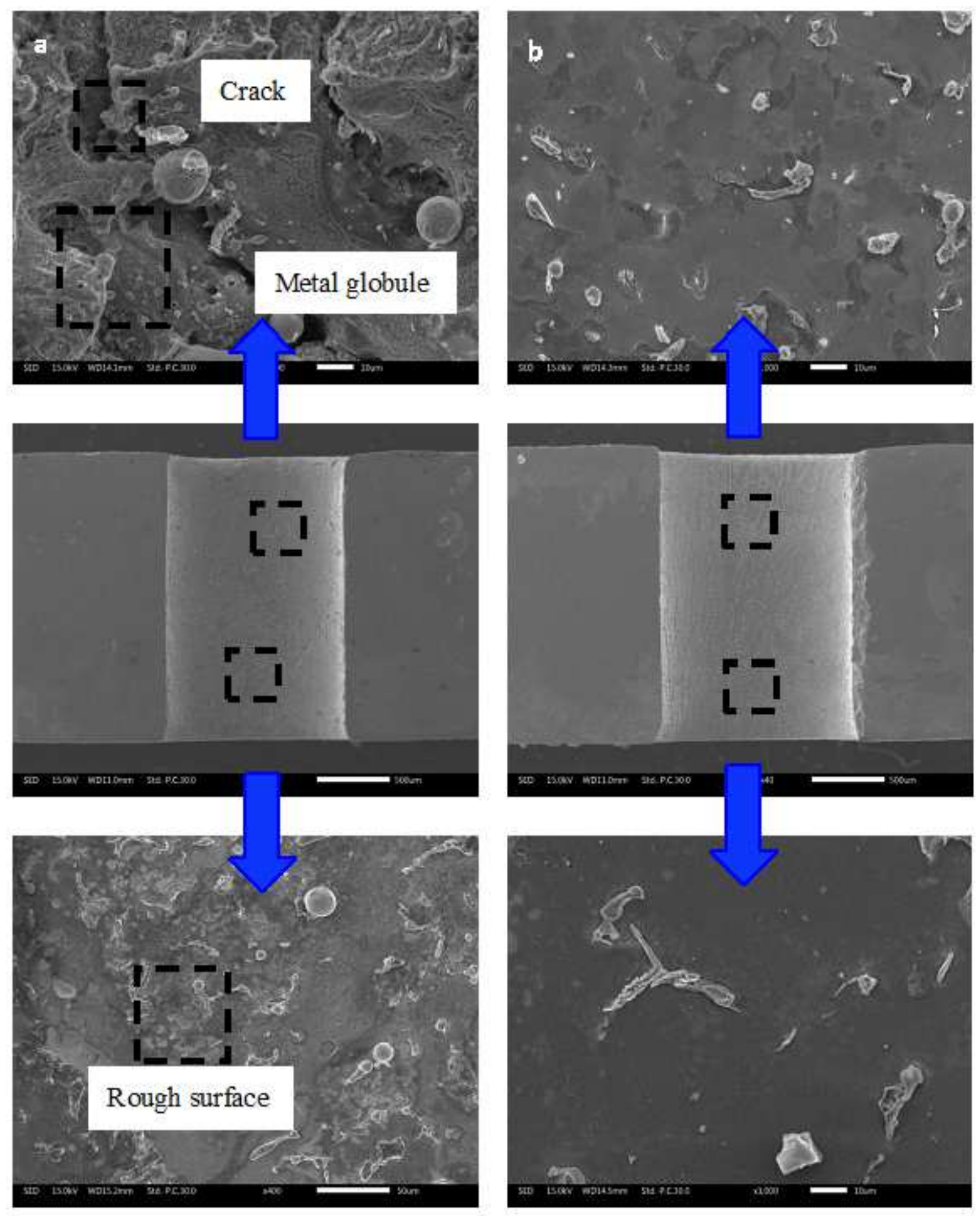

Figure 14

The morphology of holes in the wall after combined machining: (a) cylindrical electrode; (b) helical electrode 
a

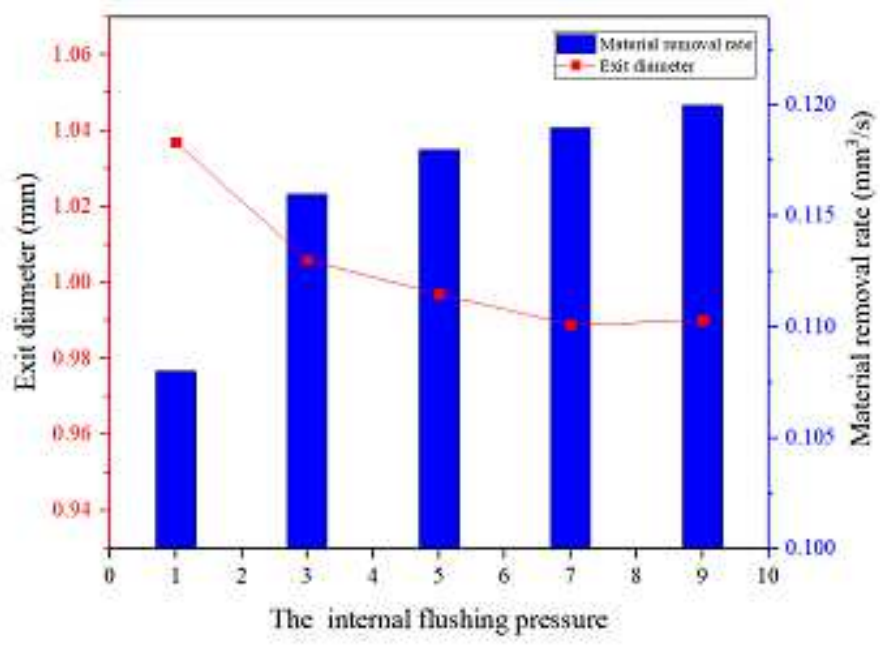

b

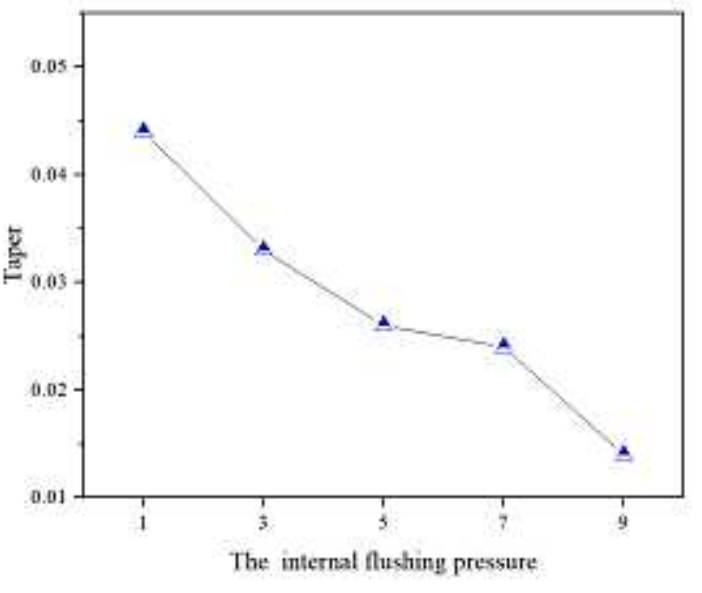

Figure 15

Influence of different internal flushing pressures on machining performance in high-speed EDM: (a) diameter of the exit and the MRR; (b) taper
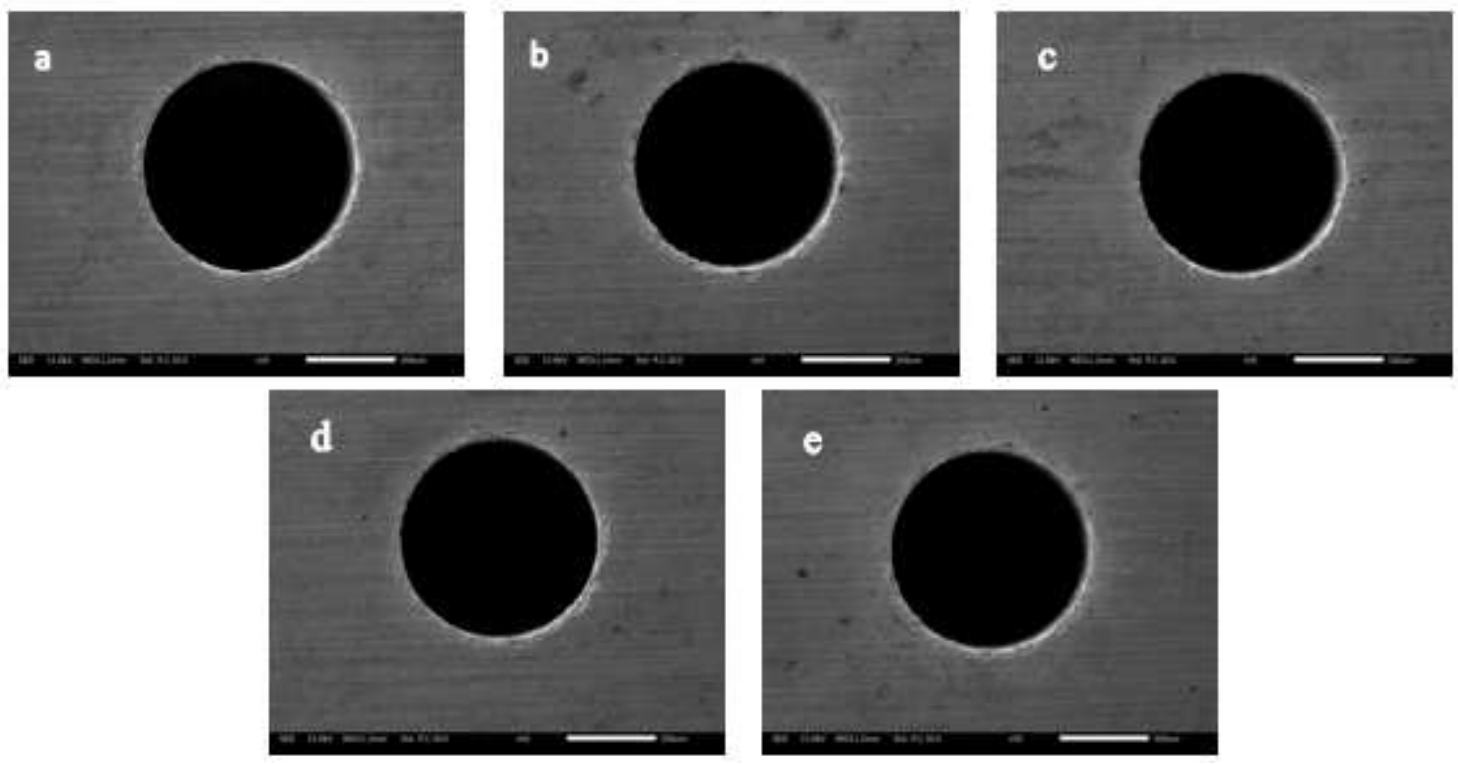

Figure 16

The morphologies of the exit in high-speed EDM: (a) $1 \mathrm{MPa}$; (b) $3 \mathrm{MPa}$; (c) $5 \mathrm{MPa}$; (d) $7 \mathrm{MPa}$; (e) $9 \mathrm{MPa}$ 

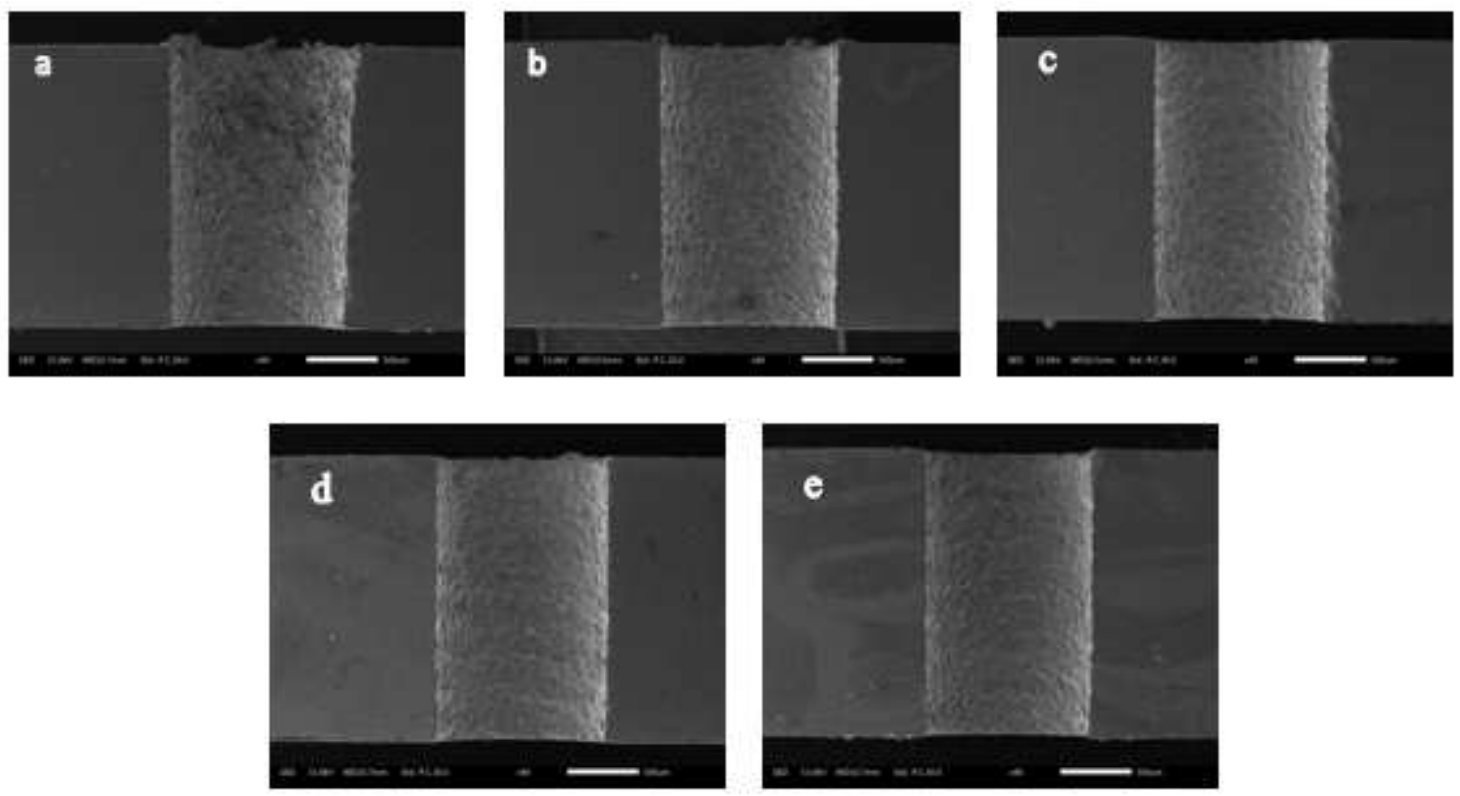

\section{Figure 17}

The morphologies of the holes in the wall in high-speed EDM: (a) $1 \mathrm{MPa}$; (b) $3 \mathrm{MPa}$; (c) $5 \mathrm{MPa}$; (d) $7 \mathrm{MPa}$; (e) $9 \mathrm{MPa}$

a

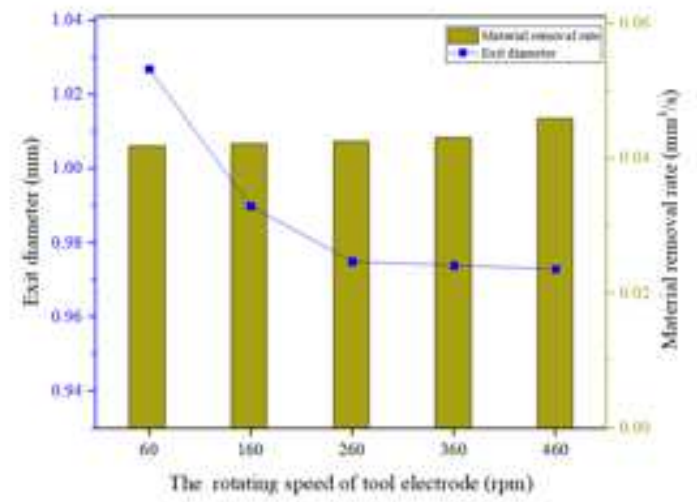

\section{b}

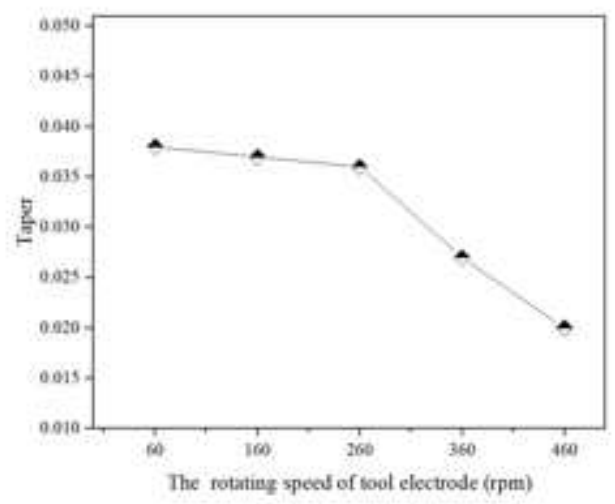

Figure 18

Influence of different rotating speeds on machining performance in high-speed EDM: (a) diameter of exit and the MRR; (b) taper 

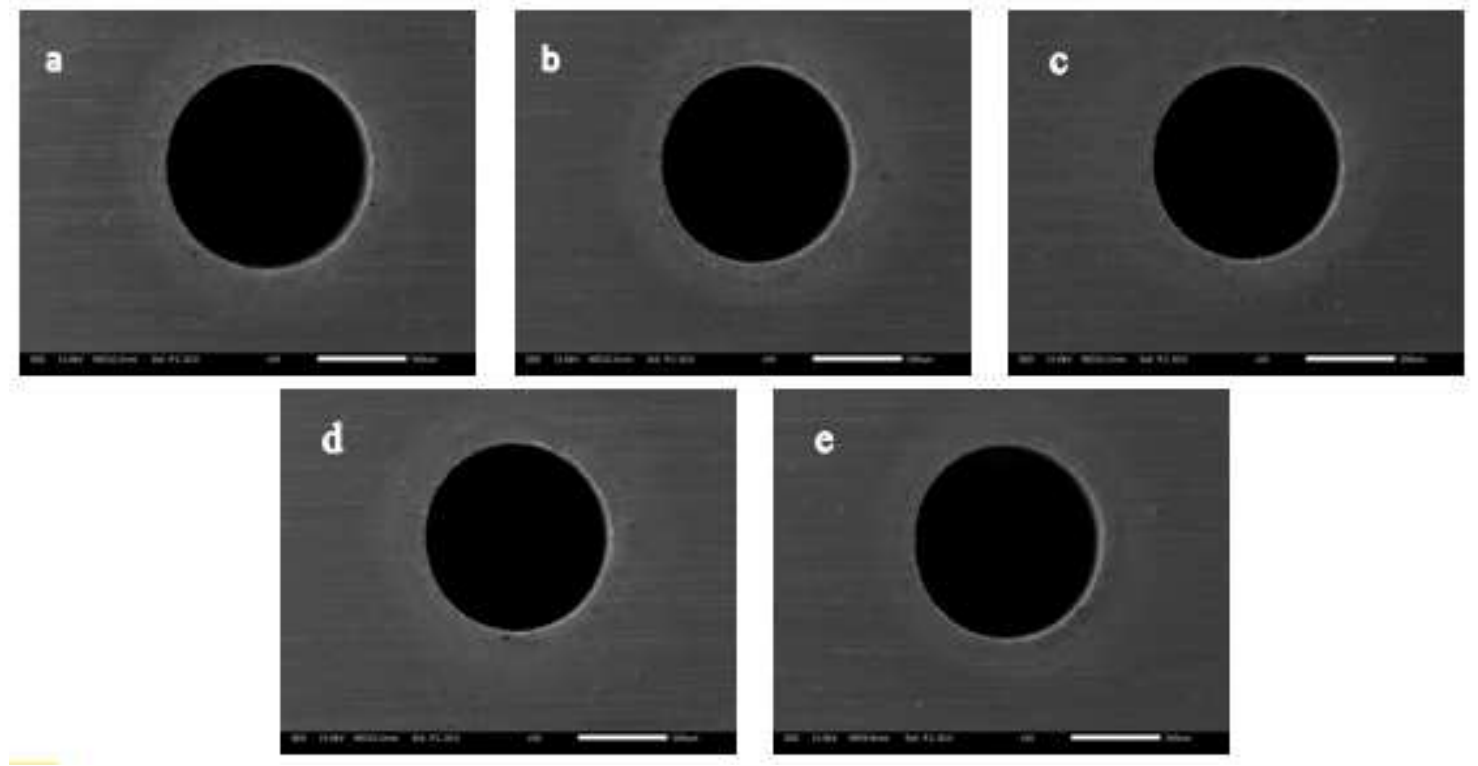

\section{Figure 19}

The morphologies of the exit at different rotating speeds of the tool electrode in high-speed EDM: (a) 60 rpm; (b) 160 rpm; (c) 260 rpm; (d) 360 rpm; (e) 460 rpm
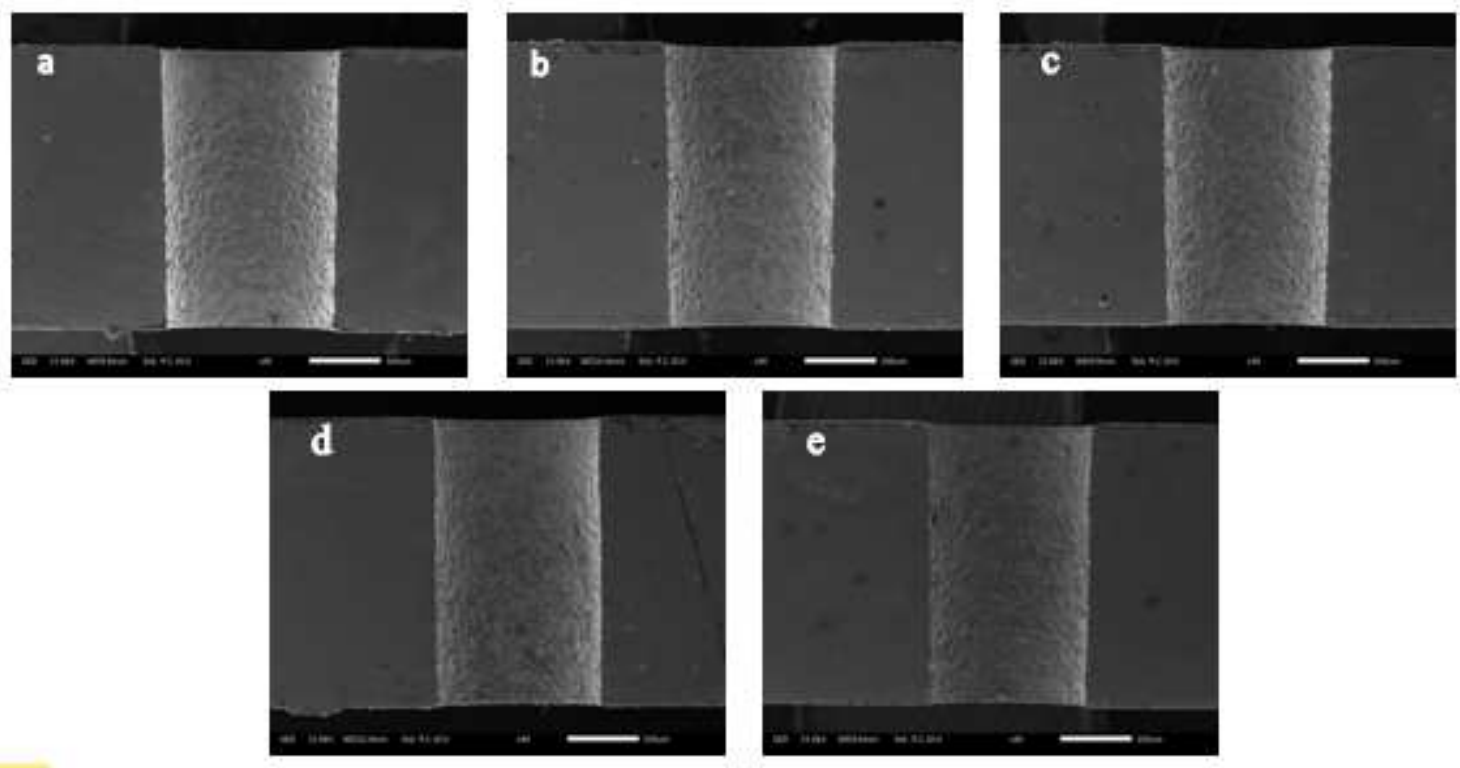

\section{Figure 20}

The morphologies of the holes in the wall at different rotating speeds of the tool electrode in high-speed EDM: (a) 60 rpm; (b) 160 rpm; (c) 260 rpm; (d) 360 rpm; (e) 460 rpm 

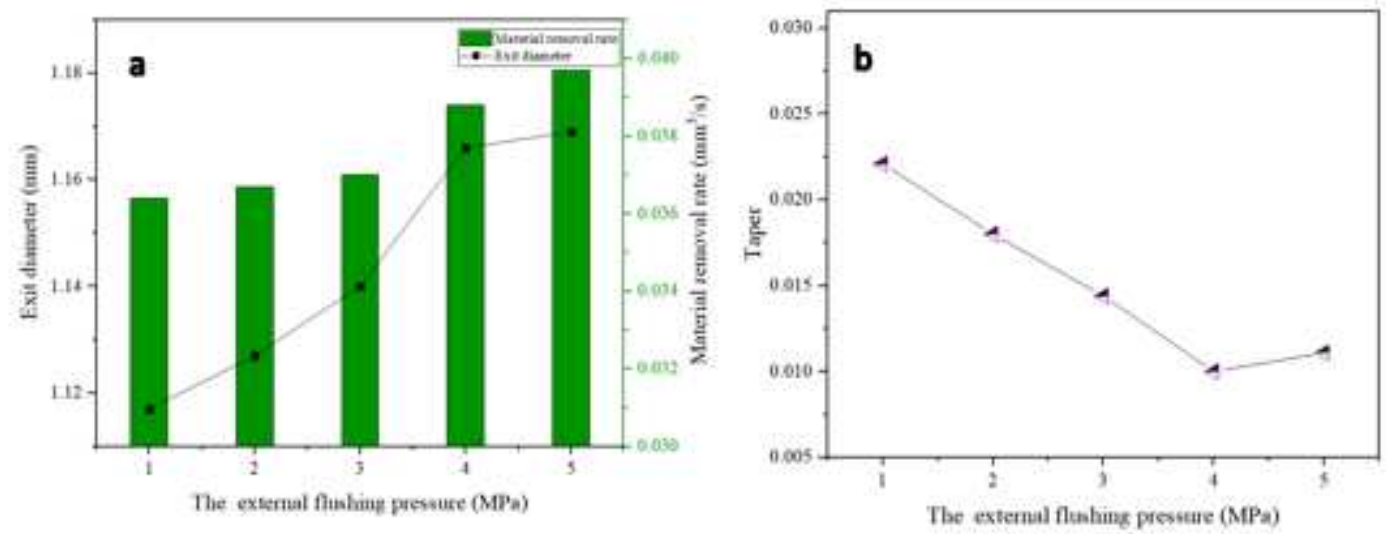

Figure 21

Influence of external flushing pressure on machining performance in ECM: (a ) diameter and MRR; (b) taper
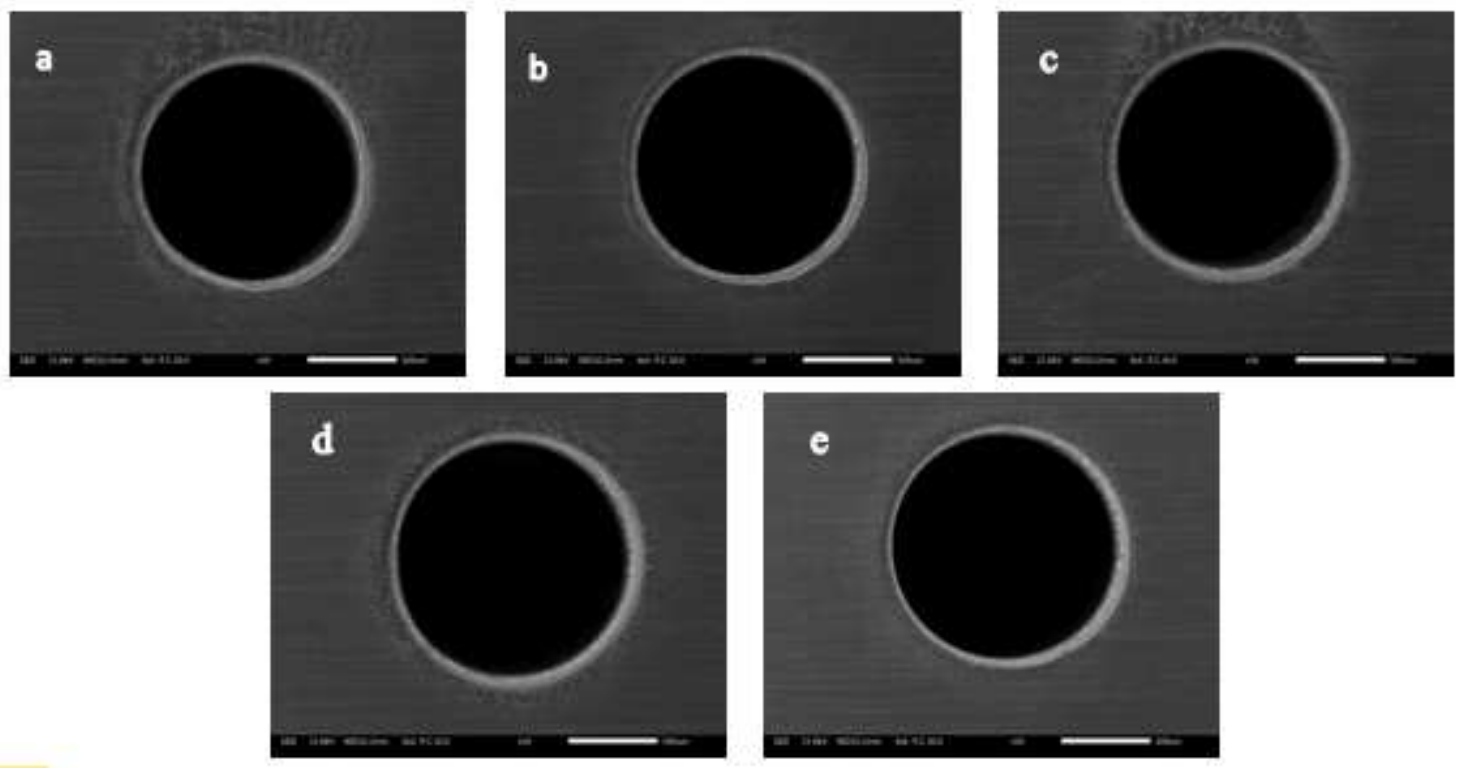

Figure 22

The morphologies of the exit with different external flush pressures in ECM: (a) $1 \mathrm{MPa}$; (b) $2 \mathrm{MPa}$; (c) 3 $\mathrm{MPa}$; (d) $4 \mathrm{MPa}$; (e) $5 \mathrm{MPa}$ 

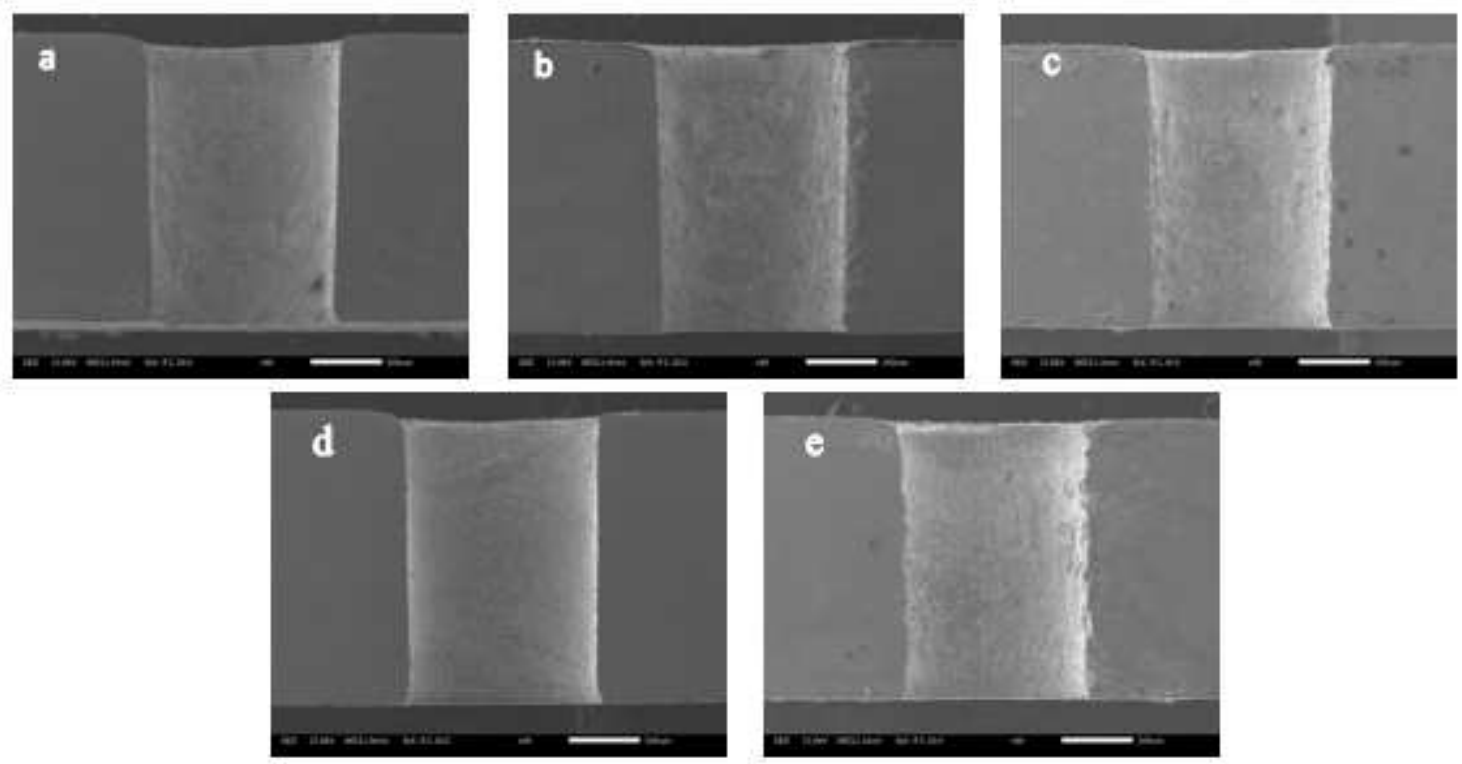

\section{Figure 23}

The morphologies of the hole in the wall with different external flush pressures in ECM: (a) $1 \mathrm{MPa}$; (b) 2 $\mathrm{MPa}$; (c) $3 \mathrm{MPa}$; (d) $4 \mathrm{MPa}$; (e) $5 \mathrm{MPa}$
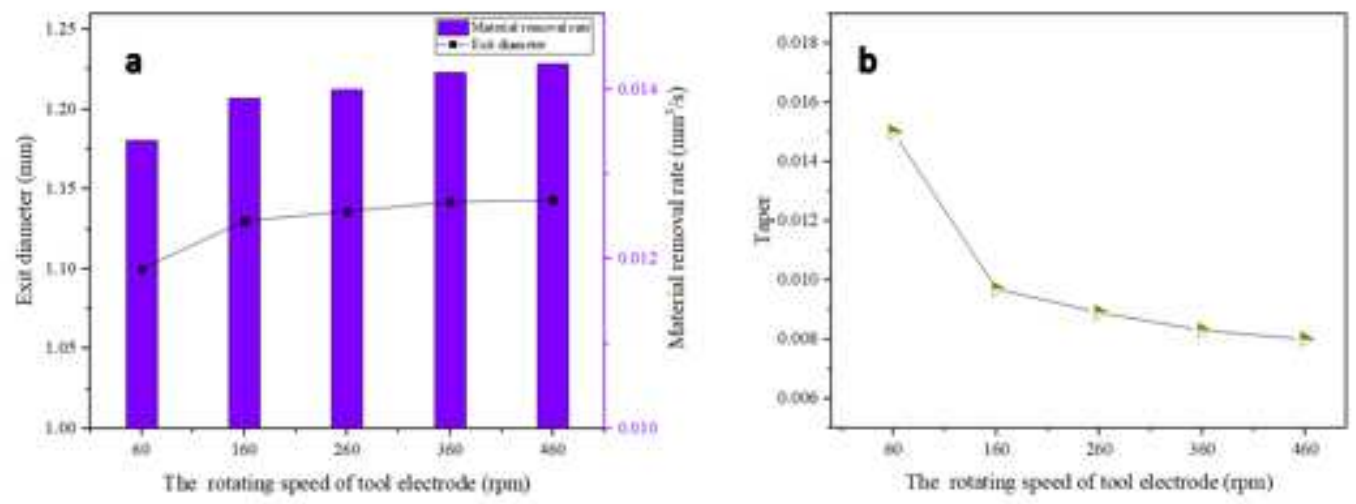

Figure 24

Influence of the rotating speed of the tool electrode on machining performance in ECM: (a) diameter and MRR; (b) taper 

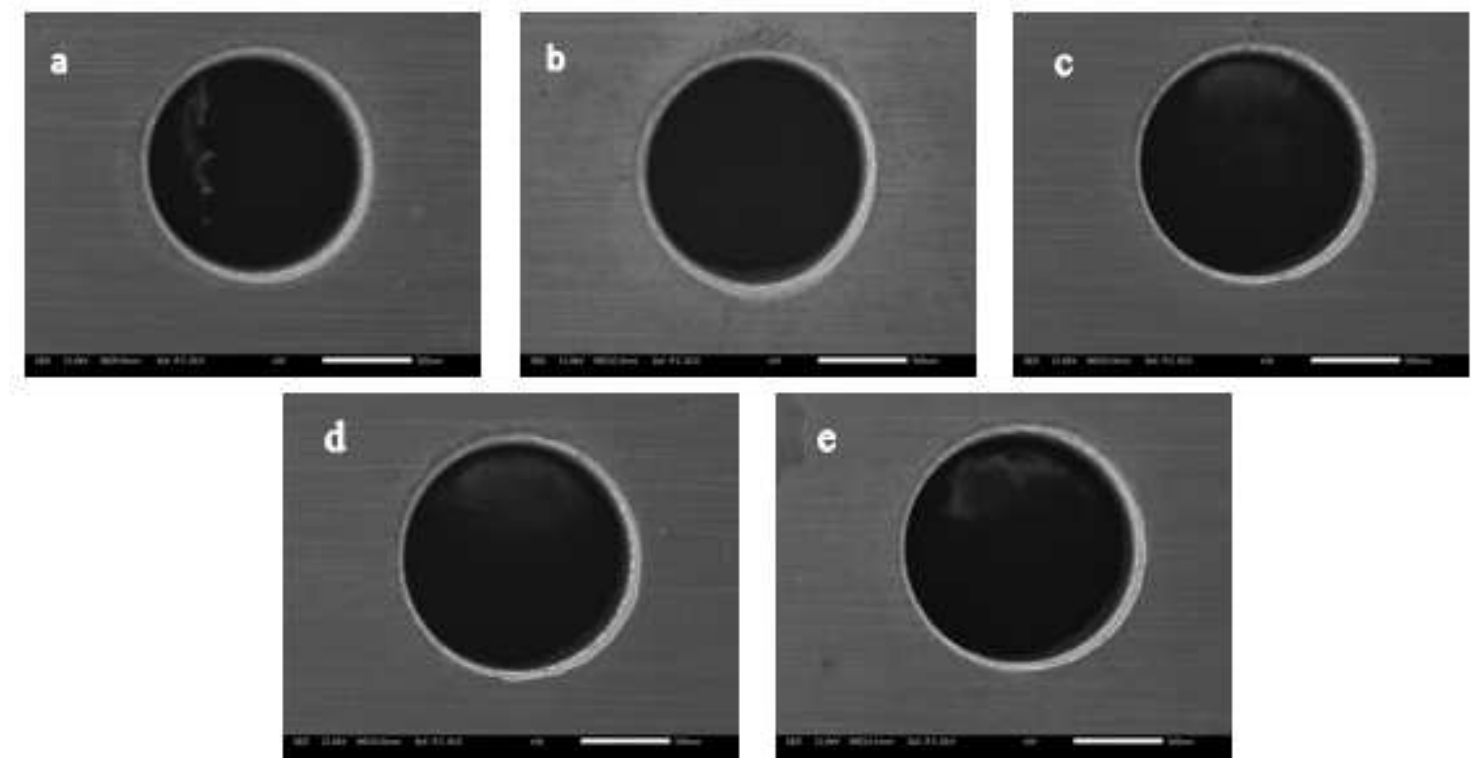

\section{Figure 25}

The morphologies of the exit at different the rotating speeds of the tool electrode in ECM: (a) $60 \mathrm{rpm}$; (b) 160 rpm; (c) 260 rpm; (d) 360 rpm; (e) 460 rpm
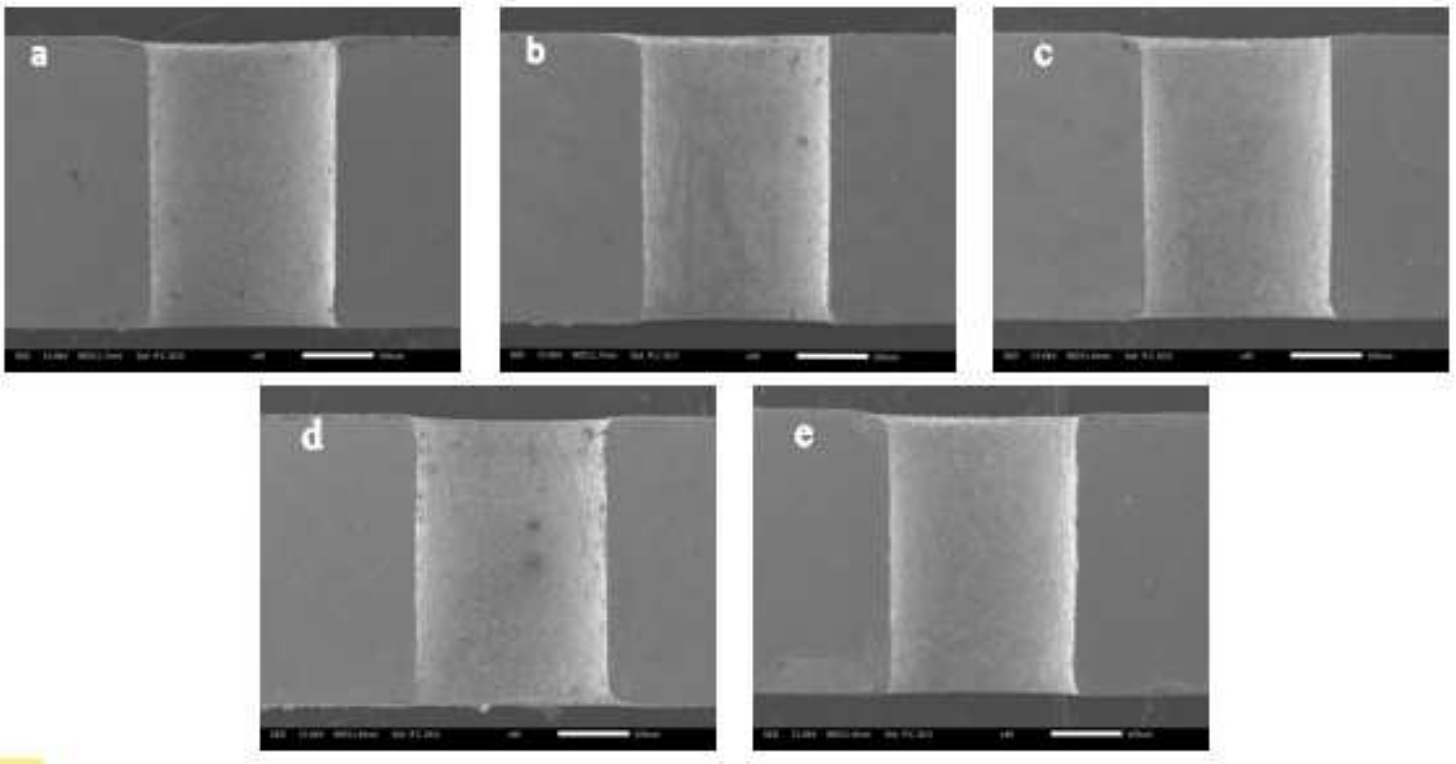

Figure 26

The morphologies of the holes in the wall at different the rotating speeds of the tool electrode in ECM: (a) 60 rpm; (b) 160 rpm; (c) 260 rpm; (d) 360 rpm; (e) 460 rpm 